\title{
Renormalization group effects in dark matter interactions
}

\author{
Fady Bishara, ${ }^{a}$ Joachim Brod, ${ }^{b}$ Benjamin Grinstein ${ }^{c}$ and Jure Zupan ${ }^{b}$ \\ ${ }^{a}$ Deutsches Elektronen-Synchrotron (DESY), \\ D-22607 Hamburg, Germany \\ ${ }^{b}$ Department of Physics, University of Cincinnati, \\ Cincinnati, Ohio 45221, U.S.A. \\ ${ }^{c}$ Department of Physics, University of California-San Diego, \\ La Jolla, CA 92093, U.S.A. \\ E-mail: fady.bishara@desy.de, brodjm@ucmail.uc.edu, \\ bgrinstein@ucsd.edu, zupanje@ucmail.uc.edu
}

ABSTRACT: We present a renormalization-group (RG) analysis of dark matter interactions with the standard model, where dark matter is allowed to be a component of an electroweak multiplet, and has a mass at or below the electroweak scale. We consider, in addition to the gauge interactions, the complete set of effective operators for dark matter interactions with the standard model above the weak scale, up to and including mass dimension six. We calculate the RG evolution of these operators from the high scale $\Lambda$ down to the weak scale, and perform the matching to the tower of effective theories below the weak scale. We also summarize the RG evolution below the weak scale and the matching to the nonrelativistic nuclear interactions. We present several numerical examples and show that in certain cases the dark matter - nucleus scattering rate can change by orders of magnitude when the electroweak running is included.

Keywords: Beyond Standard Model, Effective Field Theories, Renormalization Group

ARXIV EPRINT: 1809.03506 


\section{Contents}

1 Introduction 1

2 Effective Lagrangian above the electroweak scale $\quad 4$

2.1 Dimension-five operator basis 5

$\begin{array}{lll}2.2 & \text { Dimension-six operator basis } & 6\end{array}$

$\begin{array}{lll}3 & \text { Renormalization group running } & 7\end{array}$

3.1 Mixing of dimension five operators 8

$\begin{array}{ll}3.2 \text { Mixing of dimension six operators } & 11\end{array}$

4 Matching to EFT below the weak scale $\quad 18$

$\begin{array}{ll}4.1 \text { Light dark matter } & 20\end{array}$

4.2 Electroweak scale dark matter 23

$\begin{array}{lll}4.3 & \text { RG running below the electroweak scale } & 27\end{array}$

$\begin{array}{lll}\text { 4.4 DM interactions with nucleons and nuclei } & 28\end{array}$

5 The effects of RG running $\quad 31$

$\begin{array}{lll}5.1 & \text { Low energy phenomenology ignoring RG running } & 32\end{array}$

5.1.1 Magnetic or electric dipole operators 32

5.1.2 Operators with DM scalar currents 32

5.1.3 Operators with DM vector current and with quark vector or axialvector currents 33

5.1.4 Operators with DM axial-vector and with quark vector or axialvector currents 34

5.1.5 Operators with Higgs vector currents 35

5.2 Inclusion of RG running 36

5.2.1 Operators with DM axial-vector current and 3rd generation quark axial-vector current 37

$\begin{array}{lll}\text { 5.2.2 Vector - Axial-vector (first generation) } & 39\end{array}$

5.2.3 Vector - Axial-vector (third generation) 40

$6 \quad$ Summary and conclusions $\quad 42$

$\begin{array}{ll}\text { A Conventions and input } & 43\end{array}$

A.1 Standard model in the unbroken and broken phases 43

A.2 Numerical inputs for the electroweak running 44

B Nonrelativistic coefficients $\quad 45$

$\begin{array}{ll}\text { C SM EFT operators } & 45\end{array}$

$\begin{array}{ll}\text { D Mixing in the dark sector } & 49\end{array}$ 
E Unphysical operators $\quad \mathbf{5 0}$

E.1 Evanescent operators $\quad 51$

$\begin{array}{lll}\text { E.2 EOM - Vanishing operators } & 51\end{array}$

\section{Introduction}

For a large class of dark matter (DM) models, the physics of direct detection experiments can be described using Effective Field Theory (EFT) [1-27]. There are several scales that enter the problem: the DM mass, $m_{\chi}$, the scale of the mediators, $\Lambda$, through which the DM interacts with the visible sector, and, finally, the standard model (SM) scales - the masses of the SM particles and the scale of strong interactions, $\Lambda_{\mathrm{QCD}}$. The EFT description of DM direct detection is appropriate as long as the mediators are heavier than a few hundred $\mathrm{MeV}$, i.e., above the typical momentum exchange in direct detection experiments. Furthermore, the EFT description is necessary in order to consistently treat the hadronic physics in the scattering of DM on nuclei.

The EFT approach is especially appealing if one does not want to commit to a particular DM model when interpreting the results of direct detection experiments. The direct detection bounds can be expressed as the bounds on the coefficients of local operators, which can then be compared between different direct detection experiments in a modelindependent manner $[16,20,21,23,27]$. If the mediator scale is above the DM mass, $\Lambda \gtrsim m_{\chi}$, they can also be compared to indirect detection bounds [28-34], and to colliders searches if $\Lambda$ is above the typical partonic momentum exchange in the collision [35-43]. At the LHC the typical partonic momentum often does exceed the mediator scale, $\Lambda$, in which case one needs to resort to simplified models [44-57].

In the present manuscript we are interested in the connection between the DM theory at the mediator scale, $\Lambda$, and the EFT describing DM direct detection. To do so one needs to run through a tower of EFTs that connects the UV scale $\Lambda$ with the nuclear scale. We assume that ${ }^{1}$

$$
\Lambda \gg m_{\chi} \sim m_{Z}
$$

where $m_{Z}=91.1876 \mathrm{GeV}$ is the $Z$-boson mass. Figure 1 depicts the resulting tower of EFTs. At a particular scale $\mu$ the appropriate EFT is constructed from the relevant propagating degrees of freedom.

At $\mu \sim \Lambda$ the propagating degrees of freedom are either the full theory of DM interactions, presumably renormalizable, or a simplified model of DM interactions, including the mediators. For $\mu<\Lambda$ the mediators are integrated out, leading to an EFT with nonrenormalizable interactions between DM and the visible sector. At $\mu \sim m_{Z}$ the top quark, the Higgs, and the $W, Z$ bosons are integrated out. For $\mu<m_{Z}$ the DM interactions are therefore described by nonrenormalizable operators in an EFT that contains only

\footnotetext{
${ }^{1}$ For $\Lambda \sim m_{\chi}$ one needs to match onto an EFT with non-relativistic DM, the Heavy Dark Matter EFT (HDMET), already at the scale $\Lambda$.
} 


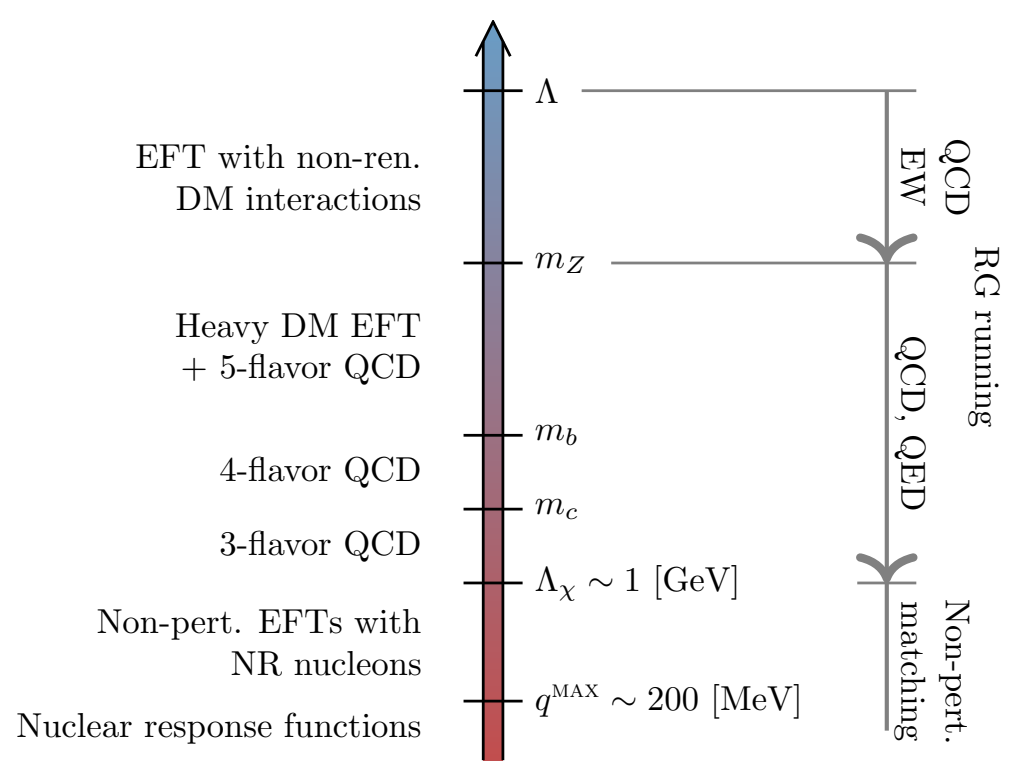

Figure 1. The tower of EFTs linking the UV scale $\Lambda$ to the scale of interactions between the nucleons and the DM.

DM (which, for $m_{\chi} \sim m_{Z}$, is now described by a nonrelativistic field), and the bottom-, charm-, strange-, down-, and up-quark, as well as the leptons, gluons and photons. At $\mu \sim m_{b}$ one integrates out the bottom quark, and at $\mu \sim m_{c}$ the charm quark. Finally, at $\mu \sim \mathcal{O}(1 \mathrm{GeV})$ a nonperturbative matching to an EFT with pions and nucleons, i.e., a chiral effective theory, is performed [18-21]. This is then used in a chiral EFT approach to nuclear forces together with the nuclear response functions to obtain the hadronic matrix element for each of the DM-nucleon interaction operators [9-13, 23, 34, 58, 59].

The EFT operators mix under the renormalization-group (RG) evolution when going from $\Lambda$ to $m_{Z}$, from $m_{Z}$ to $m_{b}$, etc. The primary purpose of this paper is to calculate the anomalous dimensions for the RG running from $\Lambda$ to $m_{Z}$ for the case of Dirac fermion DM in an arbitrary electroweak multiplet. This RG running can be phenomenologically important since it can mix operators that are velocity suppressed in the nonrelativistic limit with operators that are not velocity suppressed (see refs. [22, 60-67] for further examples of relevant loop corrections in DM interactions). In addition, we also perform the rest of the running and matching down to the nuclear level and give several numerical examples.

The possibility that DM is part of an electroweak multiplet is allowed by direct detection constraints as long as DM does not couple to the $Z$ boson at tree level (for instance, this is the case if the DM multiplet has odd dimensionality and does not carry hypercharge). The exchanges of $W, Z, h$ bosons with a quark current then generate a contribution to DM-nucleon scattering at one-loop and two-loop level, see figure 2 and refs. [68, 69]. Since these contributions are loop-suppressed and result in either a chirality flip or spin-dependent scattering, it is quite possible that the leading contribution is due to exchanges of heavy mediators. This is illustrated in figure 3 where we show for several non-renormalizable interactions at which values of the mediator mass, $\Lambda_{\text {equal }}$, the non-renormalizable and 

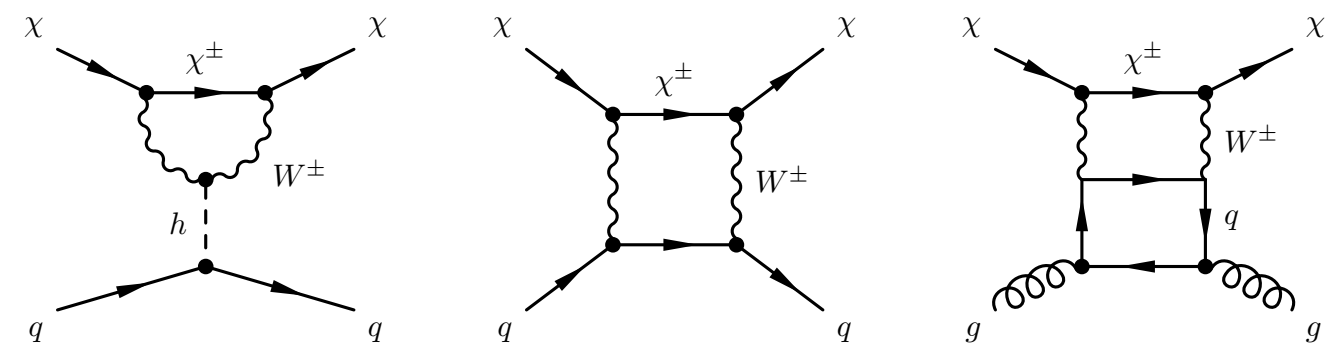

Figure 2. Representative one-loop (left and middle) and two-loop (right) diagrams contributing to the direct detection scattering of DM that is part of an electroweak multiplet.

renormalizable contributions to scattering on Xenon are equal. For mediators lighter than $\Lambda_{\text {equal }}$ the scattering rates are dominated by the non-renormalizable interactions. Even if the mediators are very heavy, many orders of magnitude heavier than the weak scale, they can still give the leading effect in spin-independent scattering. Furthermore, the operators that lead to velocity-suppressed contributions, such as vector-axial interactions, are only poorly constrained. A mixing into velocity unsuppressed, coherently enhanced operators at one-loop, two-loop, or potentially even three-loop can therefore still be the leading contribution to the scattering rate. This motivates both the use of the complete tower of EFTs and the calculation of the leading-logarithmic effects captured by RG running.

In our analysis we cover both the case of DM with electroweak-scale mass, $m_{\chi} \sim m_{Z}$, and light DM, $m_{\chi} \ll m_{Z}$. Note that we do not require DM to be a thermal relic, and therefore allow for a large range of DM masses and interactions. Above the electroweak scale we limit our analysis to a basis of operators with mass dimension five and six, and work to one-loop order for the anomalous dimensions. The matching corrections are calculated at tree level, except for the cases where one-loop contributions can be numerically important, for instance, if the matching generates gluonic operators. The subsequent RG evolution below the electroweak scale has been described in detail in refs. [16, 21]; see also ref. [23] for a computer code that implements the running numerically. Several interesting cases are left for future work, such as the case of several DM multiplets, the case of scalar DM, the case of very heavy DM, $m_{\chi} \gg m_{Z}$, as well as the analysis of higher dimension operators.

The paper is organized as follows. In section 2 we give the complete basis of dimensionfive and dimension-six operators for DM interacting with the SM, valid above the electroweak scale. The anomalous dimensions describing the mixing of these operators are presented in section 3, while in section 4 we give the matchings to the tower of EFTs below the electroweak symmetry breaking scale, and collect the results on the running down to the hadronic scale, along with the subsequent nonperturbative matching to the chiral EFT and the nuclear responses. Section 5 contains illustrative examples showcasing the effects of operator mixing on DM direct detection phenomenology. The conclusions are given in section 6. Appendix A contains our notation and conventions, appendix $\mathrm{C}$ the mixing with the pure SM operators, appendix D the mixing with the pure dark sector, and appendix E a list of unphysical operators used in the calculation. 

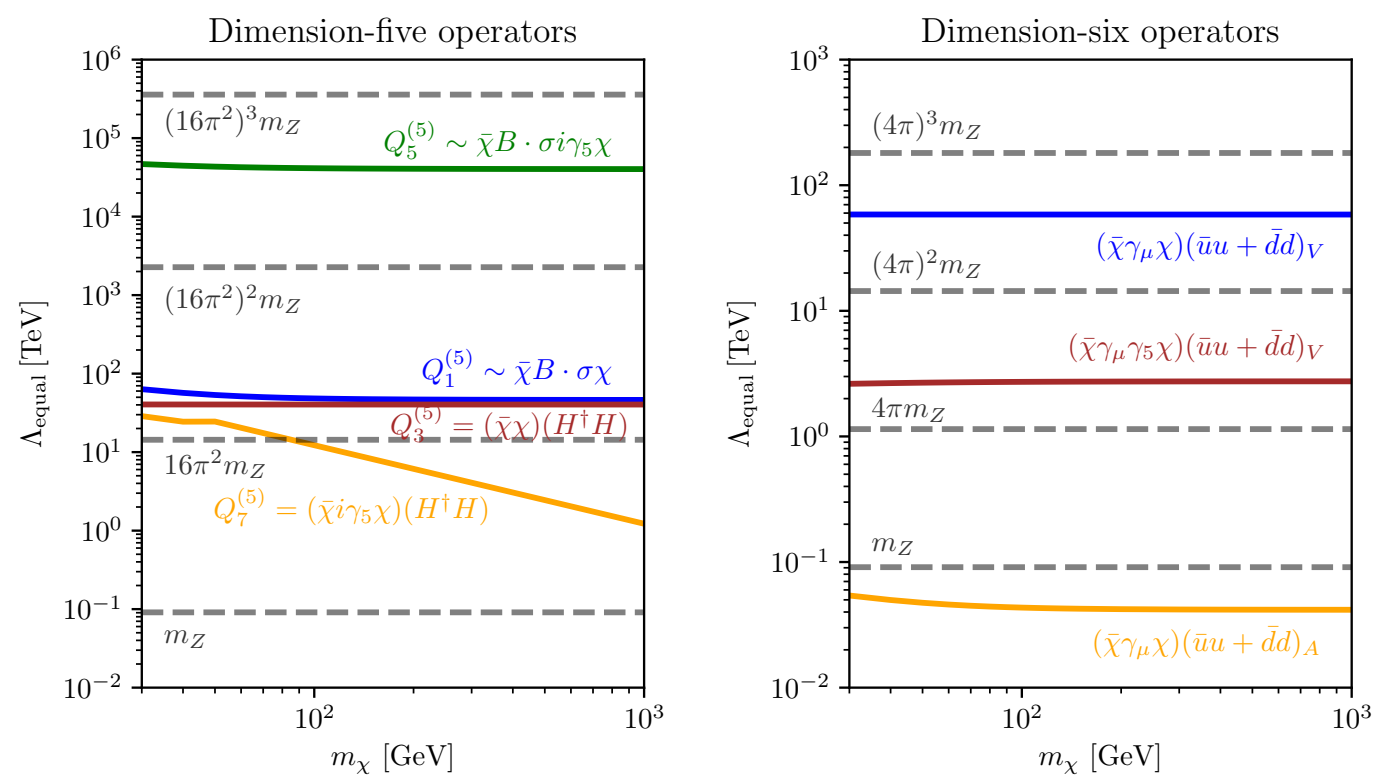

Figure 3. The contribution from the non-renormalizable operator $Q_{i}^{(d)}$ dominates in direct detection scattering over the renormalizable contributions, if the suppression scale $\Lambda$ is below the corresponding solid line (i.e., for $\Lambda=\Lambda_{\text {equal }}$ the non-renormalizable and the renormalizable contributions are of the same size). Examples shown are for triplet Dirac fermion DM with $Y_{\chi}=0$ scattering on a Xenon target, so that the contributions from renormalizable interactions start at one-loop. The dashed gray lines denote the electroweak scale, $m_{Z}$, and the scales roughly $n$ loop factors above it, $(4 \pi)^{2 n} m_{Z}$ on the left and $(4 \pi)^{n} m_{Z}$ on the right. Dimension-five operators are shown in the left panel, dimension-six operators in the right panel.

\section{Effective Lagrangian above the electroweak scale}

We extend the SM by a single Dirac fermion, a $Z_{2}$-odd electroweak multiplet of dimension $d_{\chi}=2 I_{\chi}+1$, hypercharge $Y_{\chi}$, and mass $m_{\chi}$, whose electrically neutral component is the DM. Here $I_{\chi}$ is the weak isospin of the DM multiplet (see appendix A for our conventions). One-loop electroweak corrections split the multiplet components, so that the charged particles are heavier than DM and decay in the early universe (see refs. $[7,70]$ ). In the numerical examples in section 5 we set $Y_{\chi}=0$, so that the phenomenologically dangerous tree-level vectorial $Z$ couplings are absent. In the calculation of anomalous dimension in this section we do, however, keep the $Y_{\chi}$ dependence, so that the results can be more generally applied. ${ }^{2}$

Within our set-up there are two types of DM interactions with the visible sector: either through the exchanges of SM particles, or through new states - the mediators. In general both of these contributions are present. Our default assumption is that DM has electroweak scale mass, while the mediators are much heavier, with masses of order $\Lambda \gg m_{Z}$. We thus

\footnotetext{
${ }^{2}$ This added generality is needed in the case of light DM. Collider experiments require that the charged components of the multiplet that contains the DM have electroweak scale masses. The required large splitting of the spectrum arises from mixing with additional multiplets that have to be properly accounted for in the running of Wilson coefficients below the NP scale, $\Lambda$. In our sample applications below, the light DM case is unrealistic - and given for illustration purposes only - because the effect of the required additional multiplets is ignored. Analysis of realistic examples is left for future work.
} 
have the following hierarchy of scales,

$$
\Lambda \gg m_{\chi} \sim m_{Z} \gg \Lambda_{\mathrm{QCD}} \gtrsim q,
$$

where $q \sim \mathcal{O}(100 \mathrm{MeV})$ is the typical momentum transfer in DM scattering on nuclei. We will also discuss the case of light DM, $m_{\chi} \ll m_{Z}$.

When one considers processes at energy scales below the mass of the mediators, $\mu<\Lambda$, the mediators can be integrated out. The effective DM Lagrangian, valid for $\Lambda>\mu>m_{Z}$, is given by

$$
\mathcal{L}_{\chi}=\mathcal{L}_{\chi}^{(4)}+\mathcal{L}_{\chi}^{(5)}+\mathcal{L}_{\chi}^{(6)}+\cdots,
$$

where the superscripts denote the dimensionality of the operators in the Lagrangian. The renormalizable part of the effective Lagrangian is, for a Dirac-fermion DM multiplet,

$$
\mathcal{L}_{\chi}^{(4)}=\bar{\chi} i \gamma^{\mu} D_{\mu} \chi-m_{\chi} \bar{\chi} \chi
$$

The covariant derivative comprises the interactions with the electroweak gauge bosons $W_{\mu}^{a}$ and $B_{\mu}$; see appendix A for further details on our notation. For $\mu \gg m_{Z} \sim m_{\chi}$ the mass parameter $m_{\chi}$ can effectively be set to zero.

The non-renormalizable terms in the effective Lagrangian (2.2),

$$
\mathcal{L}_{\chi}^{(5)}=\sum_{a} \frac{C_{a}^{(5)}}{\Lambda} Q_{a}^{(5)}, \quad \mathcal{L}_{\chi}^{(6)}=\sum_{a} \frac{C_{a}^{(6)}}{\Lambda^{2}} Q_{a}^{(6)}, \quad \ldots
$$

arise from integrating out the mediators. Depending on the mediator model it is possible that only $\mathcal{L}_{\chi}^{(5)}$ or only $\mathcal{L}_{\chi}^{(6)}$ are generated, but in general both will be present. We truncate the expansion at dimension six since most mediator models generate nonzero Wilson coefficients, $C_{a}^{(d)}$, in at least one of the two effective Lagrangians, $\mathcal{L}_{\chi}^{(5)}, \mathcal{L}_{\chi}^{(6)}$ (for exceptions where the first contributions arise only at dimension seven, see, e.g., refs. [71, 72]; the complete basis at dimension seven has been presented in ref. [26]). When writing the basis we assume that there is a conserved global dark $\mathrm{U}(1)_{D}$ quantum number, which forbids currents of the form $\bar{\chi}^{c} \Gamma \chi$, where $\chi^{c}$ is the charge-conjugated DM field, and $\Gamma$ denotes a generic string of Dirac matrices. (This assumption is to be relaxed in a follow-up work, where we plan to extend our analysis to the case of Majorana fermions and more than one multiplet.)

\subsection{Dimension-five operator basis}

The CP-conserving dimension-five operators are

$$
\begin{aligned}
Q_{1}^{(5)} & =\frac{g_{1}}{8 \pi^{2}}\left(\bar{\chi} \sigma^{\mu \nu} \chi\right) B_{\mu \nu}, & Q_{2}^{(5)} & =\frac{g_{2}}{8 \pi^{2}}\left(\bar{\chi} \sigma^{\mu \nu} \tilde{\tau}^{a} \chi\right) W_{\mu \nu}^{a}, \\
Q_{3}^{(5)} & =(\bar{\chi} \chi)\left(H^{\dagger} H\right), & Q_{4}^{(5)} & =\left(\bar{\chi} \tilde{\tau}^{a} \chi\right)\left(H^{\dagger} \tau^{a} H\right),
\end{aligned}
$$

while the CP-odd operators have an extra insertion of $\gamma_{5}$,

$$
\begin{aligned}
Q_{5}^{(5)} & =\frac{g_{1}}{8 \pi^{2}}\left(\bar{\chi} \sigma^{\mu \nu} i \gamma_{5} \chi\right) B_{\mu \nu}, & Q_{6}^{(5)} & =\frac{g_{2}}{8 \pi^{2}}\left(\bar{\chi} \sigma^{\mu \nu} \tilde{\tau}^{a} i \gamma_{5} \chi\right) W_{\mu \nu}^{a}, \\
Q_{7}^{(5)} & =\left(\bar{\chi} i \gamma_{5} \chi\right)\left(H^{\dagger} H\right), & Q_{8}^{(5)} & =\left(\bar{\chi} \tilde{\tau}^{a} i \gamma_{5} \chi\right)\left(H^{\dagger} \tau^{a} H\right) .
\end{aligned}
$$


Here and below, $H$ is the SM Higgs doublet, and the $\mathrm{SU}(2)$ generators $\tilde{\tau}^{a}, \tau^{a}$ are defined in appendix A. All non-displayed $\mathrm{SU}(2)$ (and, below, also color) indices are assumed to be contracted within the brackets. Note that if $\chi$ is a $\mathrm{SU}(2)$ singlet, the operators $Q_{2}^{(5)}, Q_{4}^{(5)}$, $Q_{6}^{(5)}$, and $Q_{8}^{(5)}$ are absent. In a perturbative UV theory the operators $Q_{1,2}^{(5)}$ and $Q_{5,6}^{(5)}$ are generated at one loop, while the operators $Q_{3,4}^{(5)}$ and $Q_{7,8}^{(5)}$ are typically generated at tree level. This expectation is reflected in our normalization of the operators.

\subsection{Dimension-six operator basis}

At dimension six there are many more operators. We do not consider flavor-violating operators, keeping our discussion minimal. For each SM fermion generation, $i=1,2,3$, there are then eight operators that are products of DM currents and quark currents,

$$
\begin{array}{rlrl}
Q_{1, i}^{(6)} & =\left(\bar{\chi} \gamma_{\mu} \tilde{\tau}^{a} \chi\right)\left(\bar{Q}_{L}^{i} \gamma^{\mu} \tau^{a} Q_{L}^{i}\right), & & Q_{5, i}^{(6)}=\left(\bar{\chi} \gamma_{\mu} \gamma_{5} \tilde{\tau}^{a} \chi\right)\left(\bar{Q}_{L}^{i} \gamma^{\mu} \tau^{a} Q_{L}^{i}\right), \\
Q_{2, i}^{(6)} & =\left(\bar{\chi} \gamma_{\mu} \chi\right)\left(\bar{Q}_{L}^{i} \gamma^{\mu} Q_{L}^{i}\right), & & Q_{6, i}^{(6)}=\left(\bar{\chi} \gamma_{\mu} \gamma_{5} \chi\right)\left(\bar{Q}_{L}^{i} \gamma^{\mu} Q_{L}^{i}\right), \\
Q_{3, i}^{(6)} & =\left(\bar{\chi} \gamma_{\mu} \chi\right)\left(\bar{u}_{R}^{i} \gamma^{\mu} u_{R}^{i}\right), & Q_{7, i}^{(6)}=\left(\bar{\chi} \gamma_{\mu} \gamma_{5} \chi\right)\left(\bar{u}_{R}^{i} \gamma^{\mu} u_{R}^{i}\right), \\
Q_{4, i}^{(6)}=\left(\bar{\chi} \gamma_{\mu} \chi\right)\left(\bar{d}_{R}^{i} \gamma^{\mu} d_{R}^{i}\right), & Q_{8, i}^{(6)}=\left(\bar{\chi} \gamma_{\mu} \gamma_{5} \chi\right)\left(\bar{d}_{R}^{i} \gamma^{\mu} d_{R}^{i}\right) .
\end{array}
$$

Here $Q_{L}$ denotes the left-handed quark doublet, and $u_{R}, d_{R}$ the right-handed up- and downtype quark singlets, respectively. The analogous operators involving lepton currents are

$$
\begin{aligned}
Q_{9, i}^{(6)} & =\left(\bar{\chi} \gamma_{\mu} \tilde{\tau}^{a} \chi\right)\left(\bar{L}_{L}^{i} \gamma^{\mu} \tau^{a} L_{L}^{i}\right), & & Q_{12, i}^{(6)}=\left(\bar{\chi} \gamma_{\mu} \gamma_{5} \tilde{\tau}^{a} \chi\right)\left(\bar{L}_{L}^{i} \gamma^{\mu} \tau^{a} L_{L}^{i}\right), \\
Q_{10, i}^{(6)} & =\left(\bar{\chi} \gamma_{\mu} \chi\right)\left(\bar{L}_{L}^{i} \gamma^{\mu} L_{L}^{i}\right), & & Q_{13, i}^{(6)}=\left(\bar{\chi} \gamma_{\mu} \gamma_{5} \chi\right)\left(\bar{L}_{L}^{i} \gamma^{\mu} L_{L}^{i}\right), \\
Q_{11, i}^{(6)} & =\left(\bar{\chi} \gamma_{\mu} \chi\right)\left(\bar{\ell}_{R}^{i} \gamma^{\mu} \ell_{R}^{i}\right), & Q_{14, i}^{(6)} & =\left(\bar{\chi} \gamma_{\mu} \gamma_{5} \chi\right)\left(\bar{\ell}_{R}^{i} \gamma^{\mu} \ell_{R}^{i}\right),
\end{aligned}
$$

where $L_{L}$ denotes the left-handed lepton doublet, and $\ell_{R}$ the right-handed down-type lepton singlet. Finally, there are four dimension-six operators involving Higgs currents,

$$
\begin{array}{ll}
Q_{15}^{(6)}=\left(\bar{\chi} \gamma^{\mu} \tilde{\tau}^{a} \chi\right)\left(H^{\dagger} i \stackrel{\leftrightarrow}{D}^{a}{ }_{\mu} H\right), & Q_{17}^{(6)}=\left(\bar{\chi} \gamma^{\mu} \gamma_{5} \tilde{\tau}^{a} \chi\right)\left(H^{\dagger} i \stackrel{\leftrightarrow}{D}_{\mu}{ }_{\mu} H\right), \\
Q_{16}^{(6)}=\left(\bar{\chi} \gamma^{\mu} \chi\right)\left(H^{\dagger} i \stackrel{\leftrightarrow}{D}_{\mu} H\right), & Q_{18}^{(6)}=\left(\bar{\chi} \gamma^{\mu} \gamma_{5} \chi\right)\left(H^{\dagger} i \stackrel{\leftrightarrow}{D}_{\mu} H\right) .
\end{array}
$$

The Higgs currents are defined in terms of hermitian combinations of the covariant derivatives, $\stackrel{\leftrightarrow}{D}_{\mu} \equiv D_{\mu}-\overleftarrow{D}_{\mu}^{\dagger}$ and $\stackrel{\leftrightarrow}{D}_{\mu}^{a} \equiv \tau^{a} D_{\mu}-\overleftarrow{D}_{\mu}^{\dagger} \tau^{a}$. Additional operators with covariant derivatives acting on the DM fields vanish via the DM equations of motion, up to total derivatives. As in the case of dimension-five operators, the basis simplifies if DM is a $\mathrm{SU}(2)$ singlet. In this case, the operators $Q_{1, i}^{(6)}, Q_{5, i}^{(6)}, Q_{9, i}^{(6)}, Q_{12, i}^{(6)}, Q_{15}^{(6)}$, and $Q_{17}^{(6)}$ vanish and should be dropped from the basis.

While the operators (2.5)-(2.8) and (2.9)-(2.17) mix under RG running, they do not yet form a closed set under renormalization; for this we also need to include the pure SM operators (see appendix C) and the operators with only DM fields. To the extent that we neglect the mixing of the pure DM operators among themselves, we need only four operators for our purposes, which we can choose as

$$
\begin{array}{ll}
D_{1}^{(6)}=\left(\bar{\chi} \gamma_{\mu} \chi\right)\left(\bar{\chi} \gamma^{\mu} \chi\right), & D_{2}^{(6)}=\left(\bar{\chi} \gamma_{\mu} \gamma_{5} \chi\right)\left(\bar{\chi} \gamma^{\mu} \chi\right), \\
D_{3}^{(6)}=\left(\bar{\chi} \gamma_{\mu} \tilde{\tau}^{a} \chi\right)\left(\bar{\chi} \gamma^{\mu} \tilde{\tau}^{a} \chi\right), & D_{4}^{(6)}=\left(\bar{\chi} \gamma_{\mu} \gamma_{5} \tilde{\tau}^{a} \chi\right)\left(\bar{\chi} \gamma^{\mu} \tilde{\tau}^{a} \chi\right) .
\end{array}
$$




\section{$3 \quad$ Renormalization group running}

The RG running proceeds through several sequential steps, $\Lambda \rightarrow \mu_{\mathrm{EW}} \rightarrow \mu_{b} \rightarrow \mu_{c}$, with matching thresholds at the electroweak scale, $\mu_{\mathrm{EW}}$, the bottom-quark mass scale, $\mu_{b}$, and the charm-quark mass scale, $\mu_{c}$. We first review briefly each of the steps, and then give the details in this and the subsequent section.

Running from the mediator scale, $\Lambda$, to the EW scale, $\mu_{\mathrm{EW}} \sim m_{Z} \sim m_{\chi}$, results in the mixing of the operators in the effective DM Lagrangian, eq. (2.2). We perform the calculation of the RG running using dimensional regularization in $d=4-2 \epsilon$ dimensions. Following the conventions in ref. [73], we define the anomalous dimension matrix $\gamma$ by

$$
\mu \frac{d}{d \mu} \vec{C}(\mu)=\gamma^{T} \vec{C}(\mu)
$$

where $\vec{C}$ is a vector of Wilson coefficients, ${ }^{3}$ and the superscript $T$ denotes matrix transposition. The anomalous dimension matrix receives a number of different contributions that we treat separately, so that

$$
\gamma=\frac{\alpha_{s}}{4 \pi} \gamma_{s}^{(0)}+\left(\frac{\alpha_{s}}{4 \pi}\right)^{2} \gamma_{s}^{(1)}+\frac{\alpha_{1}}{4 \pi} \gamma_{1}^{(0)}+\frac{\alpha_{2}}{4 \pi} \gamma_{2}^{(0)}+\sum_{f=t, b, c, \tau} \frac{\alpha_{f}}{4 \pi} \gamma_{y_{f}}^{(0)}+\frac{\alpha_{\lambda}}{4 \pi} \gamma_{\lambda}^{(0)}+\cdots
$$

Here, we defined $\alpha_{f} \equiv y_{f}^{2} / 4 \pi$ and $\alpha_{\lambda} \equiv \lambda / 4 \pi$, where $y_{f}$ is the Yukawa coupling of the fermion $f$ and $\lambda$ the Higgs quartic coupling (for normalizations see appendix A), while the other parameters are defined in terms of the gauge couplings in the usual way, $\alpha_{i} \equiv g_{i}^{2} / 4 \pi$. The ellipsis denotes higher-order contributions. Note that the anomalous dimension above the EW scale does not depend on the QCD coupling constant, since DM does not carry color, while all the DM-quark operators in (2.9)-(2.12) contain conserved quark currents in the limit of zero quark masses. The situation is different below the EW scale.

The solution to the RG evolution equation (3.1) gives the Wilson coefficients at any scale $\mu_{\mathrm{EW}}<\mu<\Lambda$,

$$
\vec{C}(\mu)=U(\mu, \Lambda) \vec{C}(\Lambda),
$$

where $U(\mu, \Lambda)$ is the evolution operator from $\Lambda$ to $\mu$, obtained by solving (3.1), or equivalently

$$
\frac{d}{d \ln \mu} U(\mu, \Lambda)=\gamma^{T} U(\mu, \Lambda),
$$

with the initial condition $U(\Lambda, \Lambda)=1$. The leading-order RG evolution effectively sums the terms of the form $\alpha_{i}^{n} \log ^{n}\left(\Lambda / \mu_{\mathrm{EW}}\right)$ to all orders. Since some of the anomalous dimensions are large, we count $\alpha_{i} \log \left(\Lambda / \mu_{\mathrm{EW}}\right) \sim \mathcal{O}(1)$. We work to leading-logarithmic order and thus include all terms that are $\mathcal{O}(1)$. This means that the matching conditions are calculated to the same order, i.e., are obtained at tree level. Matching is done at one-loop, if the tree level contribution vanishes and the one-loop contribution is numerically important, for details see below.

\footnotetext{
${ }^{3}$ For dimension-five operators some Wilson coefficients need to be redefined to have simple forms of anomalous dimensions, see eq. (3.8).
} 
The first matching arises at the EW scale, $\mu_{\mathrm{EW}} \sim m_{Z}$, where one integrates out the top quark, Higgs, $W$ and $Z$. For $\mu<\mu_{\mathrm{EW}}$ the propagating degrees of freedom are then the photon, the gluons, $n_{f}=5$ quark flavors, and the leptons. The RG running in the five-flavor theory is given by the anomalous dimension matrix $\gamma_{[5]}$. It receives QCD and electromagnetic contributions, so that at one loop order,

$$
\gamma_{\left[n_{f}\right]}=\frac{\alpha_{s}}{4 \pi} \gamma_{\left[n_{f}\right], s}^{(0)}+\frac{\alpha}{4 \pi} \gamma_{\left[n_{f}\right], e}^{(0)}+\cdots,
$$

where $\alpha_{s}$ and $\alpha$ are the strong and electromagnetic coupling constants. At $\mu_{b} \sim m_{b}$ the bottom quark is integrated out. The resulting four flavor EFT has as the propagating degrees of freedom the photon, gluons, leptons, and $n_{f}=4$ flavors of quarks. It is valid for $\mu_{c}<\mu<\mu_{b}$, where $\mu_{c} \sim m_{c}$ is the scale at which the charm quark and the $\tau$ lepton are integrated out. The running from $\Lambda$ down to the scale $\mu_{\text {had }} \sim 2 \mathrm{GeV}$, where the hadronic matrix elements are evaluated, can thus formally be written as

$$
\begin{aligned}
\left.\vec{C}\left(\mu_{\mathrm{had}}\right)\right|_{n_{f}=3}=U_{[3]}\left(\mu_{\mathrm{had}}, \mu_{c}\right) M_{[4 \rightarrow 3]}\left(\mu_{c}\right) U_{[4]}\left(\mu_{c}, \mu_{b}\right) M_{[5 \rightarrow 4]}\left(\mu_{b}\right) & \\
& \times U_{[5]}\left(\mu_{b}, \mu_{\mathrm{EW}}\right) M_{[\mathrm{EW} \rightarrow 5]}\left(\mu_{\mathrm{EW}}\right) U\left(\mu_{\mathrm{EW}}, \Lambda\right) \vec{C}(\Lambda) .
\end{aligned}
$$

Here $U_{\left[n_{f}\right]}\left(\mu, \mu^{\prime}\right)$ are the evolution operators from $\mu^{\prime}$ to $\mu$ in a theory with $n_{f}$ quark flavors that satisfy an evolution equation similar to (3.4),

$$
\frac{d}{d \ln \mu} U_{\left[n_{f}\right]}\left(\mu, \mu^{\prime}\right)=\gamma_{\left[n_{f}\right]}^{T} U_{\left[n_{f}\right]}\left(\mu, \mu^{\prime}\right) .
$$

In the numerics we take $\mu_{\text {had }} \sim \mu_{c} \sim 2 \mathrm{GeV}$, and thus set $U_{[3]}\left(\mu_{\text {had }}, \mu_{c}\right)=1$. The $M_{\left[n_{f} \rightarrow n_{f}-1\right]}$ in eq. (3.6) are the matching matrices when going from a theory with $n_{f}$ quark to a theory with $n_{f}-1$ quarks, while $M_{[\mathrm{EW} \rightarrow 5]}\left(\mu_{\mathrm{EW}}\right)$ symbolises the matching to the five-flavor theory at the EW scale. On the left side of eq. (3.6) we have denoted explicitly that the final Wilson coefficients are in the theory with only three flavors of quarks, i.e., with just $u, d$, and $s$ quarks, along with gluons, photons and the light leptons.

In the remaining part of this section we present the explicit form of the anomalous dimension matrix, eq. (3.2), that describes the mixing of the operators due to the RG evolution from the mediator scale $\Lambda$ to $\mu_{\mathrm{EW}}$. The subsequent matching and RG evolutions below the weak scale is given in section 4 . We work in the limit of flavor conservation, setting the Cabibbo-Kobayashi-Maskawa (CKM) matrix to unity. Furthermore, in this section we keep only the top, bottom, charm, and tau Yukawa couplings nonzero.

For the computation of the anomalous dimensions we used two independent automated setups. In the first, the amplitudes were generated using qgraf [74] and the anomalous dimensions were computed using the computer algebra system form [75]. The second setup relied on Mathematica packages: the Feynman rules were generated using FeynRules [76], the amplitudes with FeynArts [77], and the anomalous dimensions were computed using FormCalc [78]. A large part of the calculations were also checked using pen and paper.

\subsection{Mixing of dimension five operators}

We start by providing the anomalous dimension matrices for mixing of the $\mathrm{CP}$ conserving dimension-five operators $Q_{1}^{(5)}, \ldots, Q_{8}^{(5)}$, defined in eqs. (2.5)-(2.8). For the column of the 
dimension-five Wilson coefficients entering the RG evolution equation (3.3) we use the rescaled Wilson coefficients

$$
\vec{C}^{\prime}=\left(\frac{\alpha_{1}}{2 \pi} C_{1}^{(5)}, \frac{\alpha_{2}}{2 \pi} C_{2}^{(5)}, C_{3}^{(5)}, C_{4}^{(5)}, \frac{\alpha_{1}}{2 \pi} C_{5}^{(5)}, \frac{\alpha_{2}}{2 \pi} C_{6}^{(5)}, C_{7}^{(5)}, C_{8}^{(5)}\right) .
$$

The explicit factors of $\alpha_{1,2} / 2 \pi=g_{1,2}^{2} / 8 \pi^{2}$ in $\vec{C}^{\prime}$ ensure that the anomalous dimension matrices $\gamma_{i}^{(0)}$, still defined by (3.2), do not depend on coupling constants. The evolution of the primed Wilson coefficients is given by the analogue of eq. (3.1), namely,

$$
\mu \frac{d}{d \mu} \vec{C}^{\prime}(\mu)=\gamma^{T} \vec{C}^{\prime}(\mu)
$$

The corresponding rescaled operators are also denoted by a prime and read

$$
\begin{aligned}
Q_{1}^{\prime(5)} & =\frac{1}{g_{1}}\left(\bar{\chi} \sigma^{\mu \nu} \chi\right) B_{\mu \nu}, & Q_{2}^{\prime(5)} & =\frac{1}{g_{2}}\left(\bar{\chi} \sigma^{\mu \nu} \tilde{\tau}^{a} \chi\right) W_{\mu \nu}^{a}, \\
Q_{5}^{\prime(5)} & =\frac{1}{g_{1}}\left(\bar{\chi} \sigma^{\mu \nu} i \gamma_{5} \chi\right) B_{\mu \nu}, & Q_{6}^{\prime(5)} & =\frac{1}{g_{2}}\left(\bar{\chi} \sigma^{\mu \nu} i \gamma_{5} \tilde{\tau}^{a} \chi\right) W_{\mu \nu}^{a},
\end{aligned}
$$

while $Q_{i}^{\prime(5)} \equiv Q_{i}^{(5)}$ for $i=3,4,7,8$.

The anomalous dimension for $Q_{1}^{\prime(5)}, \ldots, Q_{8}^{\prime(5)}$ splits into two blocks, for the CP even operators, $Q_{1}^{\prime(5)}, \ldots, Q_{4}^{\prime(5)}$, and the CP odd operators, $Q_{5}^{\prime(5)}, \ldots, Q_{8}^{\prime(5)}$, while there is no mixing between the two blocks. The QCD anomalous dimensions vanish, since all fields are color neutral. The remaining one-loop anomalous dimensions for the $Q_{1}^{\prime(5)}, \ldots, Q_{4}^{\prime(5)}$ block are

$$
\begin{aligned}
{\left[\gamma_{1}^{(0)}\right]_{Q_{1 \ldots 4}^{\prime(5)} \times Q_{1 \ldots 4}^{\prime(5)}} } & =\left(\begin{array}{cccc}
\frac{5}{2} Y_{\chi}^{2}-2 \beta_{1}^{(0)} & 0 & -6 Y_{\chi} & 0 \\
-4 Y_{\chi} \mathcal{J}_{\chi} & \frac{1}{2} Y_{\chi}^{2} & 0 & 12 Y_{\chi} \\
0 & 0 & -\frac{3}{2}-\frac{3}{2} Y_{\chi}^{2} & 0 \\
0 & 0 & 0 & -\frac{3}{2}-\frac{3}{2} Y_{\chi}^{2}
\end{array}\right) \\
{\left[\gamma_{2}^{(0)}\right]_{Q_{1 \ldots 4}^{\prime(5)} \times Q_{1 \ldots 4}^{\prime(5)}} } & =\left(\begin{array}{ccccc}
2 \mathcal{J}_{\chi} & -4 Y_{\chi} & 0 & -24 \\
0 & 10 \mathcal{J}_{\chi}-8-2 \beta_{2}^{(0)} & 12 \mathcal{J}_{\chi} & 0 \\
0 & 0 & -6 \mathcal{J}_{\chi}-\frac{9}{2} & 0 \\
0 & 0 & 0 & -6 \mathcal{J}_{\chi}+\frac{3}{2}
\end{array}\right)
\end{aligned}
$$

Here $\mathcal{J}_{\chi}=I_{\chi}\left(I_{\chi}+1\right)$, with $d_{\chi}=2 I_{\chi}+1$ the dimensionality of the DM electroweak multiplet, and $Y_{\chi}$ its hypercharge. The $\beta$ functions for the gauge couplings $g_{1}$ and $g_{2}$ are given by

$$
\beta_{1}^{(0)}=-\frac{41}{6}-\frac{Y_{\chi}^{2}}{3} d_{\chi} N_{\chi}, \quad \beta_{2}^{(0)}=\frac{19}{6}-\frac{4}{9} \mathcal{J}_{\chi} d_{\chi} N_{\chi},
$$

respectively, where $N_{\chi}$ is the number of DM multiplets in the representation $I_{\chi}$ (we will mostly take $N_{\chi}=1$ ). The anomalous dimension matrices for the CP-odd operators $Q_{5}^{\prime(5)}, \ldots, Q_{8}^{\prime(5)}$ are also given by the same matrices, $\left[\gamma_{i}^{(0)}\right]_{Q_{5}^{\prime(5)} \times Q_{5}^{\prime \ldots 8}}=\left[\gamma_{i}^{(0)}\right]_{Q_{1 \cdots 4}^{\prime(5)} \times Q_{1 \cdots 4}^{\prime(5)}}$, $i=1,2, y, \lambda$, as required by the fact that $\mathrm{CP}$ breaking is not probed by the relevant one-loop diagrams. 

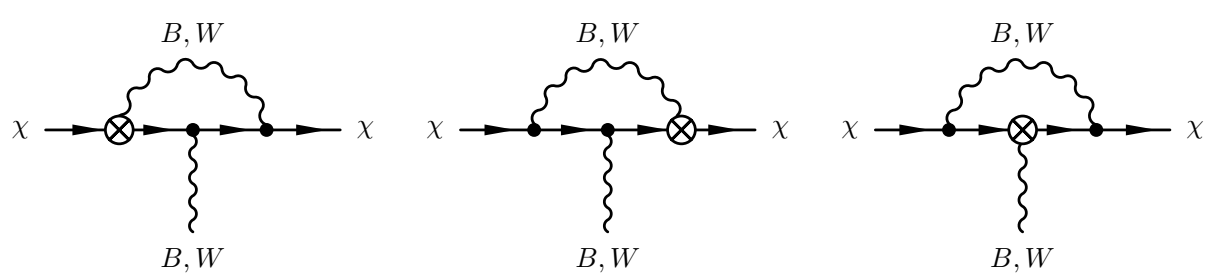

Figure 4. Sample diagrams for renormalization of $Q_{1}^{\prime(5)}, Q_{2}^{\prime(5)}$ operators due to $B_{\mu}, W_{\mu}^{a}$ exchanges at one loop.
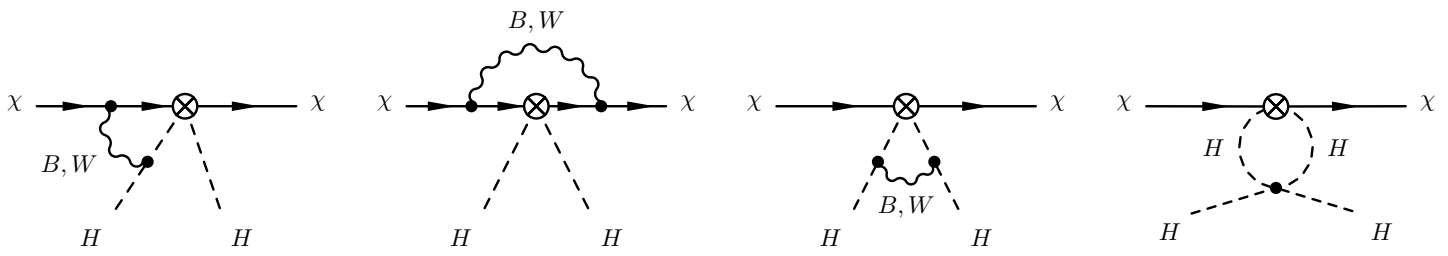

Figure 5. The renormalization of the $Q_{3}^{\prime(5)}, Q_{4}^{\prime(5)}$ operators at one loop, with only one representative of each class of diagrams shown.

The anomalous dimensions $\gamma_{1}^{(0)}$ in eq. (3.12) and $\gamma_{2}^{(0)}$ in eq. (3.13) come from the exchanges of the $B_{\mu}$ and $W_{\mu}^{a}$ gauge bosons in figures 4,5 , and 6 , respectively. They are almost diagonal, with only six nonzero off-diagonal entries. The $Q_{1}^{\prime(5)}$ mixing into $Q_{2}^{\prime(5)}$ in $\gamma_{1}^{(0)}$ is due to a loop exchange of $B_{\mu}$ with an emission of $W_{\mu}^{a}$, shown in the left two diagrams in figure 4 , while the $Q_{2}^{\prime(5)}$ mixing into $Q_{1}^{\prime(5)}$ is due to a similar diagram with $B_{\mu}$ and $W_{\mu}^{a}$ exchanged. The $Q_{1}^{\prime(5)}$ mixing into $Q_{2}^{\prime(5)}$ in $\gamma_{2}^{(0)}$ is due to a loop exchange of $B_{\mu}$ in the last diagram in figure 4 . The mixings of dipole operators, $Q_{1,2}^{\prime(5)}$, into the Higgs current operators, $Q_{3,4}^{\prime(5)}$, arise from the diagrams in figure 6 . These mixing contributions vanish for singlet $\mathrm{DM}\left(Y_{\chi}=\mathcal{J}_{\chi}=0\right)$. This is true also for the mixing of $Q_{1}^{\prime(5)}$ into $Q_{4}^{(5)}$ in $\gamma_{2}^{(0)}$ (recall that the operators $Q_{2}^{\prime(5)}$ and $Q_{4}^{\prime(5)}$ are absent for singlet DM). The contributions proportional to Yukawa couplings and the Higgs self coupling to the anomalous dimension lead only to multiplicative renormalization of $Q_{3}^{\prime(5)}$ and $Q_{4}^{\prime(5)}$ :

$$
\begin{aligned}
& {\left[\gamma_{y_{t}}^{(0)}\right]_{Q_{1 \ldots 4}^{\prime(5)} \times Q_{1 \cdots 4}^{\prime(5)}}=\left[\gamma_{y_{b}}^{(0)}\right]_{Q_{1 \cdots 4}^{\prime(5)} \times Q_{1 \cdots 4}^{(5)}}=\left[\gamma_{y_{c}}^{(0)}\right]_{Q_{1 \cdots 4}^{\prime(5)} \times Q_{1 \cdots 4}^{\prime(5)}}=\operatorname{diag}(0,0,6,6)} \\
& {\left[\gamma_{y_{\tau}}^{(0)}\right]_{Q_{1 \cdots 4}^{\prime(5)} \times Q_{1 \cdots 4}^{\prime(5)}}=\operatorname{diag}(0,0,2,2)} \\
& {\left[\gamma_{\lambda}^{(0)}\right]_{Q_{1 \cdots 4}^{\prime(5)} \times Q_{1 \cdots 4}^{\prime(5)}}=\operatorname{diag}(0,0,3,1)}
\end{aligned}
$$

They arise from the Higgs wave function renormalization, and in the case of $\gamma_{\lambda}^{(0)}$, from the last diagram in figure 5 . 

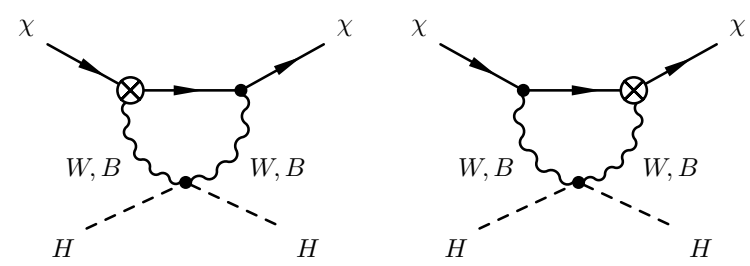

Figure 6. Sample diagrams for mixing of the dipole operators $Q_{1}^{\prime(5)}$ and $Q_{2}^{\prime(5)}$ into the Higgs operators $Q_{3}^{(5)}$ and $Q_{4}^{\prime(5)}$.

After running from $\mu \sim \Lambda$ to $\mu \sim m_{Z}$, we revert the rescaling of the Wilson coefficients, i.e.,

$$
\vec{C}=\left(\frac{2 \pi}{\alpha_{1}} C_{1}^{\prime(5)}, \frac{2 \pi}{\alpha_{2}} C_{2}^{\prime(5)}, C_{3}^{\prime(5)}, C_{4}^{\prime(5)}, \frac{2 \pi}{\alpha_{1}} C_{5}^{\prime(5)}, \frac{2 \pi}{\alpha_{2}} C_{6}^{\prime(5)}, C_{7}^{\prime(5)}, C_{8}^{\prime(5)}\right),
$$

corresponding to our original definition of operators in eqs. (2.5)-(2.8). We use the unprimed Wilson coefficients for determining the matching conditions in section 4 .

\subsection{Mixing of dimension six operators}

We turn next to the anomalous dimensions for the dimension-six operators. Counting the three SM fermion generations and keeping only flavor-diagonal fermion currents, there are 46 operators in total that couple DM with the SM. We work in the limit of flavor conservation which simplifies the structure of the anomalous dimensions.

We split the $46 \times 46$ matrix of anomalous dimensions into several sub-blocks. They correspond to three groups of operators: the operators with quark currents, $Q_{1, i}^{(6)}, \ldots, Q_{8, i}^{(6)}$; the operators with lepton current, $Q_{9, i}^{(6)}, \ldots, Q_{14, i}^{(6)}$; and the operators with Higgs currents, $Q_{15}^{(6)}, \ldots, Q_{18}^{(6)}$ (see eqs. (2.9)-(2.17) for definitions). Moreover, we will distinguish between mixing within one fermion generation, and mixing between different generations.

A technical remark is in order. To project the one-loop matrix elements onto our operator basis within the context of dimensional regularization, we have to manipulate Dirac $\gamma$ matrices in $d \neq 4$ dimensions. Strictly speaking, this requires the extension of the operator basis by evanescent operators. However, the one-loop anomalous dimensions are not affected by the choice of the evanescent operator basis, and we can effectively use four-dimensional Dirac algebra [79].

We start with the mixing among the operators that are products of DM and quark currents, $Q_{1, i}^{(6)}, \ldots, Q_{8, i}^{(6)}$, eqs. (2.9)-(2.12), within the same quark generation. The corresponding $8 \times 8$ block of the anomalous dimension matrix is given by

$$
\left[\gamma_{1}^{(0)}\right]_{Q_{1, i \cdots 8, i}^{(6)} \times Q_{1, i \cdots 8, i}^{(6)}}=\left(\begin{array}{cccccccc}
0 & 0 & 0 & 0 & -Y_{\chi} & 0 & 0 & 0 \\
0 & \frac{2}{3} d_{\chi} Y_{\chi}^{2}+\frac{2}{9} & \frac{8}{9} & -\frac{4}{9} & 0 & -Y_{\chi} & 0 & 0 \\
0 & \frac{4}{9} & \frac{2}{3} d_{\chi} Y_{\chi}^{2}+\frac{16}{9} & -\frac{8}{9} & 0 & 0 & 4 Y_{\chi} & 0 \\
0 & -\frac{2}{9} & -\frac{8}{9} & \frac{2}{3} d_{\chi} Y_{\chi}^{2}+\frac{4}{9} & 0 & 0 & 0 & -2 Y_{\chi} \\
-Y_{\chi} & 0 & 0 & 0 & 0 & 0 & 0 & 0 \\
0 & -Y_{\chi} & 0 & 0 & 0 & \frac{2}{9} & \frac{8}{9} & -\frac{4}{9} \\
0 & 0 & 4 Y_{\chi} & 0 & 0 & \frac{4}{9} & \frac{16}{9} & -\frac{8}{9} \\
0 & 0 & 0 & -2 Y_{\chi} & 0 & -\frac{2}{9} & -\frac{8}{9} & \frac{4}{9}
\end{array}\right),
$$



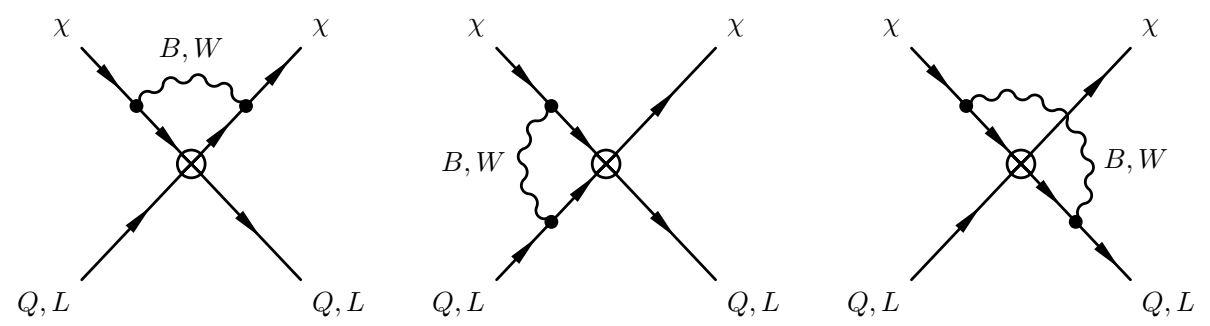

Figure 7. Sample diagrams for the renormalization of $Q_{1, i}^{(6)}, \ldots, Q_{14, i}^{(6)}$ operators due to the exchange of $B_{\mu}, W_{\mu}^{a}$ at one loop (right-handed quarks and leptons can also be on the external lines).
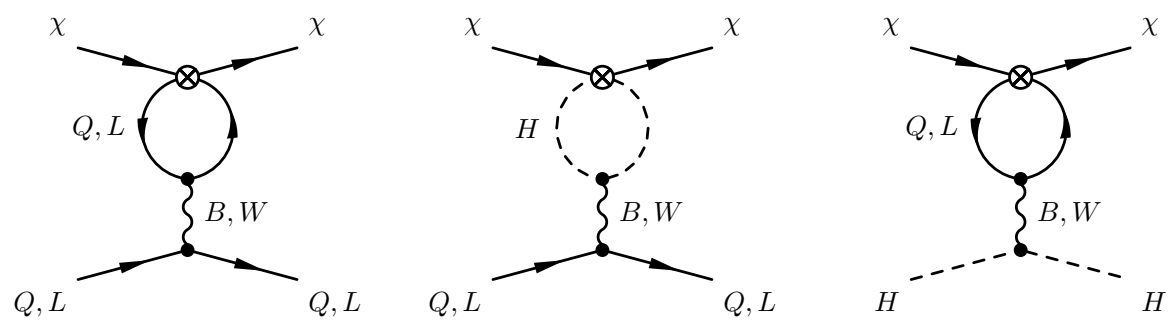

Figure 8. The renormalization of the operators $Q_{1, i}^{(6)}, \ldots, Q_{14, i}^{(6)}$ and $Q_{15}^{(6)}, \ldots, Q_{18}^{(6)}$ due to the $B_{\mu}, W_{\mu}^{a}$ penguin insertion (right-handed quarks and leptons can also be on the external lines).

for the part of the anomalous dimension matrix proportional to $g_{1}^{2}$, while the part of the anomalous dimension proportional to $g_{2}^{2}$ is

$$
\left[\gamma_{2}^{(0)}\right]_{Q_{1, i \cdots, i}^{(6)} \times Q_{1, i \cdots 8, i}^{(6)}}=\left(\begin{array}{cccccccc}
\frac{8}{9} \mathcal{J}_{\chi} d_{\chi}-4 & 0 & 0 & 0 & 0 & -3 \mathcal{J}_{\chi} & 0 & 0 \\
0 & 0 & 0 & 0 & -12 & 0 & 0 & 0 \\
0 & 0 & 0 & 0 & 0 & 0 & 0 & 0 \\
0 & 0 & 0 & 0 & 0 & 0 & 0 & 0 \\
0 & -3 \mathcal{J}_{\chi} & 0 & 0 & -4 & 0 & 0 & 0 \\
-12 & 0 & 0 & 0 & 0 & 0 & 0 & 0 \\
0 & 0 & 0 & 0 & 0 & 0 & 0 & 0 \\
0 & 0 & 0 & 0 & 0 & 0 & 0 & 0
\end{array}\right)
$$

Both of the anomalous dimension matrices are diagonal in flavor indices. As far as the $\mathrm{U}(1)$ gauge interaction is concerned, for $Y_{\chi}=0$ the operators $Q_{i}^{(6)}, i=2,3,4,6,7,8$, are partially conserved currents, and one would naively expect their anomalous dimensions to vanish. That this is not the case can be understood as the result of a non-multiplicative renormalization, allowed for U(1) gauge groups; see ref. [80]. Similar arguments apply for the QED anomalous dimensions discussed in section 4.3.

The Feynman diagrams that lead to nonzero entries in the two matrices are given in figures 7 and 8, with contributions from gauge boson exchanges between fermion lines, and penguin diagrams, respectively. We see that an exchange of the hypercharge boson $B$ between the DM and quark lines, shown in figure 7 (middle and right panel), mixes $Q_{1, i}^{(6)}$ and $Q_{5, i}^{(6)}$, while these operators do not mix with any of the remaining operators. The same contributions also mix $Q_{2, i}^{(6)}$ and $Q_{6, i}^{(6)}, Q_{3, i}^{(6)}$ and $Q_{7, i}^{(6)}$, and $Q_{4, i}^{(6)}$ and $Q_{8, i}^{(6)}$, respectively. These 
diagrams are nonzero only for DM with $\mathrm{EW}$ charges. If DM is EW neutral, the $8 \times 8$ part of $\gamma_{1}^{(0)}$ splits into two remaining $3 \times 3$ nonzero blocks formed by operators $Q_{2,3,4}^{(6)}$ and $Q_{6,7,8}^{(6)}$.

In contrast to $\gamma_{1}^{(0)}$ there are only a few nonzero entries in $\gamma_{2}^{(0)}$ in this $8 \times 8$ block. The operator $Q_{1, i}^{(6)}$ gets renormalized through diagrams in figure 7 , and mixes into $Q_{6, i}^{(6)}$ through the middle and rightmost diagrams in figure 7. Equivalent diagrams mix $Q_{2, i}^{(6)}$ and $Q_{5, i}^{(6)}$. Note that these contributions to the mixing vanish, if DM is EW neutral, while the operators $Q_{1, i}^{(6)}$ and $Q_{4, i}^{(6)}$ would be absent.

The penguin insertions, figure 8 , also lead to mixing between operators involving quark currents of different generations. The corresponding anomalous dimensions are given, for $i \neq j$, by

$$
\left[\gamma_{1}^{(0)}\right]_{Q_{2, i \ldots 4, i}^{(6)} \times Q_{2, j \cdots 4, j}^{(6)}}=\left[\gamma_{1}^{(0)}\right]_{Q_{6, i \cdots 8, i}^{(6)} \times Q_{6, j \cdots 8, j}^{(6)}}=\left(\begin{array}{ccc}
\frac{2}{9} & \frac{8}{9} & -\frac{4}{9} \\
\frac{4}{9} & \frac{16}{9} & -\frac{8}{9} \\
-\frac{2}{9} & -\frac{8}{9} & \frac{4}{9}
\end{array}\right)
$$

for the part of the anomalous dimension matrix proportional to $g_{1}^{2}$, while the part of the anomalous dimension proportional to $g_{2}^{2}$ has the following non-zero entries for $i \neq j$

$$
\left[\gamma_{2}^{(0)}\right]_{Q_{1, i}^{(6)} Q_{1, j}^{(6)}}=\left[\gamma_{2}^{(0)}\right]_{Q_{5, i}^{(6)} Q_{5, j}^{(6)}}=2
$$

All the other entries vanish.

We turn next to the $6 \times 6$ block of the anomalous dimension matrix that describes the mixing of the lepton operators $Q_{9, i}^{(6)}, \ldots, Q_{14, i}^{(6)}$, eqs. (2.13)-(2.15), among themselves, giving

$$
\left[\gamma_{1}^{(0)}\right]_{Q_{9, i \cdots 14, i}^{(6)} \times Q_{9, i \cdots 14, i}^{(6)}}=\left(\begin{array}{cccccc}
0 & 0 & 0 & 3 Y_{\chi} & 0 & 0 \\
0 & \frac{2}{3} d_{\chi} Y_{\chi}^{2}+\frac{2}{3} & \frac{4}{3} & 0 & 3 Y_{\chi} & 0 \\
0 & \frac{2}{3} & \frac{2}{3} d_{\chi} Y_{\chi}^{2} \frac{4}{3} & 0 & 0 & -6 Y_{\chi} \\
3 Y_{\chi} & 0 & 0 & 0 & 0 & 0 \\
0 & 3 Y_{\chi} & 0 & 0 & \frac{2}{3} & \frac{4}{3} \\
0 & 0 & -6 Y_{\chi} & 0 & \frac{2}{3} & \frac{4}{3}
\end{array}\right)
$$

and

$$
\left[\gamma_{2}^{(0)}\right]_{Q_{9, i \cdots 14, i}^{(6)} \times Q_{9, i \cdots 14, i}^{(6)}}=\left(\begin{array}{cccccc}
\frac{8}{9} \mathcal{J}_{\chi} d_{\chi}-\frac{16}{3} & 0 & 0 & 0 & -3 \mathcal{J}_{\chi} & 0 \\
0 & 0 & 0 & -12 & 0 & 0 \\
0 & 0 & 0 & 0 & 0 & 0 \\
0 & -3 \mathcal{J}_{\chi} & 0 & -\frac{16}{3} & 0 & 0 \\
-12 & 0 & 0 & 0 & 0 & 0 \\
0 & 0 & 0 & 0 & 0 & 0
\end{array}\right)
$$

The latter two anomalous dimension matrices are straightforward modifications of the ones for the DM-quark operators in (3.19), (3.20), taking into account different EW charges of the leptons, compared to the quarks, and the fact that there is no right-handed neutrino in the SM. (The operators containing the right-handed neutrino could be included, if necessary, and would not mix with the operators in our basis.) 
Penguin-type insertions lead to mixing between different generations also for leptons, giving (for $i \neq j$ )

$$
\left[\gamma_{1}^{(0)}\right]_{\left(Q_{10, i}^{(6)}, Q_{11, j}^{(6)}\right) \times\left(Q_{10, i}^{(6)}, Q_{11, j}^{(6)}\right)}=\left[\gamma_{1}^{(0)}\right]_{\left(Q_{13, i}^{(6)}, Q_{14, j}^{(6)}\right) \times\left(Q_{13, i}^{(6)}, Q_{14, j}^{(6)}\right)}=\left(\begin{array}{cc}
\frac{2}{3} & \frac{4}{3} \\
\frac{2}{3} & \frac{4}{3}
\end{array}\right),
$$

and

$$
\left[\gamma_{2}^{(0)}\right]_{Q_{9, i}^{(6)} Q_{9, j}^{(6)}}=\left[\gamma_{2}^{(0)}\right]_{Q_{12, i}^{(6)} Q_{12, j}^{(6)}}=\frac{2}{3}
$$

All the other entries vanish.

A very interesting effect of the one-loop RG running is that the penguin diagrams mix the operators with quark- and operators with lepton currents. This is shown in figure 8 (left), where the two quark lines coming from the EFT operator are contracted into a loop, while the emission of a $B$ converts this into a lepton current. Conversely, an operator with a leptonic current can be converted to a DM-quark operator at one-loop. The corresponding mixing of the quark operators $Q_{1}^{(6)}, \ldots, Q_{8}^{(6)}$ into the lepton operators $Q_{9}^{(6)}, \ldots, Q_{14}^{(6)}$ is given by the following $8 \times 6$ block of $\gamma_{1}^{(0)}$, now for arbitrary generation indices $i, j$

$$
\left[\gamma_{1}^{(0)}\right]_{Q_{1, i \cdots 8, i}^{(6)} \times Q_{9, j \cdots 14, j}^{(6)}}=\left(\begin{array}{cccccc}
0 & 0 & 0 & 0 & 0 & 0 \\
0 & -\frac{2}{3} & -\frac{4}{3} & 0 & 0 & 0 \\
0 & -\frac{4}{3} & -\frac{8}{3} & 0 & 0 & 0 \\
0 & \frac{2}{3} & \frac{4}{3} & 0 & 0 & 0 \\
0 & 0 & 0 & 0 & 0 & 0 \\
0 & 0 & 0 & 0 & -\frac{2}{3} & -\frac{4}{3} \\
0 & 0 & 0 & 0 & -\frac{4}{3} & -\frac{8}{3} \\
0 & 0 & 0 & 0 & \frac{2}{3} & \frac{4}{3}
\end{array}\right)
$$

The corresponding block of the $\gamma_{2}^{(0)}$ matrix has only two nonzero entries,

$$
\left[\gamma_{2}^{(0)}\right]_{Q_{1, i}^{(6)} Q_{9, j}^{(6)}}=\left[\gamma_{2}^{(0)}\right]_{Q_{5, i}^{(6)} Q_{12, j}^{(6)}}=2,
$$

while the remaining entries in this $8 \times 6$ block of $\gamma_{2}^{(0)}$ are zero.

The mixing of the lepton operators, $Q_{9}^{(6)}, \ldots, Q_{14}^{(6)}$, into the quark operators, $Q_{1}^{(6)}, \ldots, Q_{8}^{(6)}$, is given for arbitrary generation indices $i, j$ by the following $6 \times 8$ block of the $\gamma_{1}^{(0)}$ anomalous matrix

$$
\left[\gamma_{1}^{(0)}\right]_{Q_{9, i \cdots 14, i}^{(6)} \times Q_{1, j \cdots 8, j}^{(6)}}=\left(\begin{array}{cccccccc}
0 & 0 & 0 & 0 & 0 & 0 & 0 & 0 \\
0 & -\frac{2}{9} & -\frac{8}{9} & \frac{4}{9} & 0 & 0 & 0 & 0 \\
0 & -\frac{2}{9} & -\frac{8}{9} & \frac{4}{9} & 0 & 0 & 0 & 0 \\
0 & 0 & 0 & 0 & 0 & 0 & 0 & 0 \\
0 & 0 & 0 & 0 & 0 & -\frac{2}{9} & -\frac{8}{9} & \frac{4}{9} \\
0 & 0 & 0 & 0 & 0 & -\frac{2}{9} & -\frac{8}{9} & \frac{4}{9}
\end{array}\right) .
$$

The corresponding $6 \times 8$ block of the $\gamma_{2}^{(0)}$ anomalous matrix has only two nonzero entries,

$$
\left[\gamma_{2}^{(0)}\right]_{Q_{9, i}^{(6)} Q_{1, j}^{(6)}}=\left[\gamma_{2}^{(0)}\right]_{Q_{12, i}^{(6)} Q_{5, j}^{(6)}}=\frac{2}{3}
$$



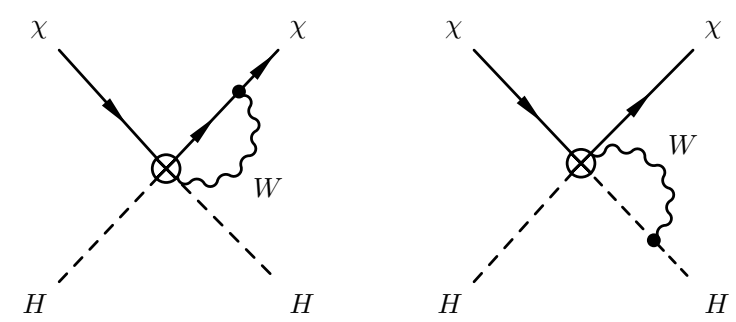

Figure 9. Additional diagrams for the renormalization of the $Q_{15}^{(6)}, \ldots, Q_{18}^{(6)}$ operators at one loop. These diagrams are in addition to the set of diagrams in figure 5 which, at dimension six, include an additional derivative w.r.t. to the dimension-five operators.

The mixing of DM-quark and DM-lepton operators, eqs. (3.27)-(3.30), has important phenomenological consequences. One implication is that, in any theory where one introduces DM-quark interactions, one-loop mixing will generate DM-lepton interactions. The converse is also true: a theory of purely "leptophilic" DM is impossible. An interaction between DM and leptons will lead to an interaction between DM and quarks via one-loop mixing. Note that the mixing is nonzero irrespective of whether or not DM carries any electroweak charge. Penguin insertions will also generate DM-quark and DM-lepton interactions, when initially only the pure DM operators (eq. (2.18)) are present; see appendix D.

Finally, we move to the mixing of dimension-six operators with Higgs currents, $Q_{15}^{(6)}, \ldots, Q_{18}^{(6)}$, eqs. (2.16)-(2.17). We start with the $4 \times 4$ blocks of the anomalous dimension matrices that give the mixing of these operators among themselves,

$$
\begin{aligned}
& {\left[\gamma_{1}^{(0)}\right]_{Q_{15 \cdots 18}^{(6)} \times Q_{15 \cdots 18}^{(6)}}=\operatorname{diag}\left(0, \frac{1}{3}+\frac{2}{3} d_{\chi} Y_{\chi}^{2}, 0, \frac{1}{3}\right)} \\
& {\left[\gamma_{2}^{(0)}\right]_{Q_{15 \cdots 18}^{(6)} \times Q_{15 \cdots 18}^{(6)}}=\operatorname{diag}\left(\frac{8}{9} \mathcal{J}_{\chi} d_{\chi}-\frac{17}{3}, 0,-\frac{17}{3}, 0\right) .}
\end{aligned}
$$

The relevant diagrams are shown in figures 5 and 9 . The renormalization induced by these contributions is multiplicative and does not lead to mixing of the DM-Higgs operators.

In addition there is mixing of the operators with quark and lepton currents into the Higgs-current operators and vice versa (see figure 8). The resulting mixing of the DMquark operators, $Q_{1, i}^{(6)}, \ldots, Q_{8, i}^{(6)}$, and the lepton operators $Q_{9, i}^{(6)}, \ldots, Q_{14, i}^{(6)}$ into the DM-Higgs operators, $Q_{15}^{(6)}, \ldots, Q_{18}^{(6)}$, are given by the following $8 \times 4$ and $6 \times 4$ blocks in the $\gamma_{1}^{(0)}$ anomalous dimension matrix $(i=1,2,3)$, respectively,

$$
\left[\gamma_{1}^{(0)}\right]_{Q_{1, i \ldots 8, i}^{(6)} \times Q_{15 \ldots 18}^{(6)}}=\left(\begin{array}{cccc}
0 & 0 & 0 & 0 \\
0 & \frac{2}{3} & 0 & 0 \\
0 & \frac{4}{3} & 0 & 0 \\
0 & -\frac{2}{3} & 0 & 0 \\
0 & 0 & 0 & 0 \\
0 & 0 & 0 & \frac{2}{3} \\
0 & 0 & 0 & \frac{4}{3} \\
0 & 0 & 0 & -\frac{2}{3}
\end{array}\right), \quad\left[\gamma_{1}^{(0)}\right]_{Q_{9, i \cdots 1, i}^{(6)} \times Q_{15 \cdots 18}^{(6)}}=\left(\begin{array}{cccc}
0 & 0 & 0 & 0 \\
0 & -\frac{2}{3} & 0 & 0 \\
0 & -\frac{2}{3} & 0 & 0 \\
0 & 0 & 0 & 0 \\
0 & 0 & 0 & -\frac{2}{3} \\
0 & 0 & 0 & -\frac{2}{3}
\end{array}\right),
$$


and by the corresponding $8 \times 4$ and $6 \times 4$ blocks in the $\gamma_{2}^{(0)}$ anomalous dimension matrix, which, however, only have two nonzero entries each,

$$
\left[\gamma_{2}^{(0)}\right]_{Q_{1, i}^{(6)} Q_{15}^{(6)}}=\left[\gamma_{2}^{(0)}\right]_{Q_{5, i}^{(6)} Q_{17}^{(6)}}=2, \quad\left[\gamma_{2}^{(0)}\right]_{Q_{9, i}^{(6)} Q_{15}^{(6)}}=\left[\gamma_{2}^{(0)}\right]_{Q_{12, i}^{(6)} Q_{17}^{(6)}}=\frac{2}{3}
$$

The mixing of the DM-Higgs operators, $Q_{15}^{(6)}, \ldots, Q_{18}^{(6)}$, into the DM-quark operators, $Q_{1}^{(6)}, \ldots, Q_{8}^{(6)}$, and into the DM-lepton operators, $Q_{9}^{(6)}, \ldots, Q_{14}^{(6)}$, is given by

$$
\left[\gamma_{1}^{(0)}\right]_{Q_{15 \cdots 18}^{(6)} \times Q_{1, i \cdots 8, i}^{(6)}}=\left(\begin{array}{cccccccc}
0 & 0 & 0 & 0 & 0 & 0 & 0 & 0 \\
0 & \frac{1}{9} & \frac{4}{9} & -\frac{2}{9} & 0 & 0 & 0 & 0 \\
0 & 0 & 0 & 0 & 0 & 0 & 0 & 0 \\
0 & 0 & 0 & 0 & 0 & \frac{1}{9} & \frac{4}{9} & -\frac{2}{9}
\end{array}\right)
$$

and

$$
\left[\gamma_{1}^{(0)}\right]_{Q_{15 \cdots 18}^{(6)} \times Q_{9, i \cdots 14, i}^{(6)}}=\left(\begin{array}{cccccc}
0 & 0 & 0 & 0 & 0 & 0 \\
0 & -\frac{1}{3} & -\frac{2}{3} & 0 & 0 & 0 \\
0 & 0 & 0 & 0 & 0 & 0 \\
0 & 0 & 0 & 0 & -\frac{1}{3} & -\frac{2}{3}
\end{array}\right)
$$

respectively, for the corresponding blocks of $\gamma_{1}^{(0)}$, while the nonzero $\gamma_{2}^{(0)}$ entries are given by

$$
\left[\gamma_{2}^{(0)}\right]_{Q_{15}^{(6)} Q_{1, i}^{(6)}}=\left[\gamma_{2}^{(0)}\right]_{Q_{17}^{(6)} Q_{5, i}^{(6)}}=\left[\gamma_{2}^{(0)}\right]_{Q_{15}^{(6)} Q_{9, i}^{(6)}}=\left[\gamma_{2}^{(0)}\right]_{Q_{17}^{(6)} Q_{12, i}^{(6)}}=\frac{1}{3} .
$$

Note that both the mixing of DM-quark and DM-lepton operators into the DM-Higgs ones and vice versa is present even if the DM does not carry any electroweak charge.

For the third-generation DM-quark operators, $Q_{1,3}^{(6)}, \ldots Q_{8,3}^{(6)}$, there is also the renormalization due to the Yukawa interaction with the Higgs (we neglect all the Yukawa interactions except with the third fermion generation and the charm Yukawa), giving

$$
\left[\gamma_{y_{c}}^{(0)}\right]_{Q_{1,2 \cdots 8,2}^{(6)} \times Q_{1,2 \cdots 8,2}^{(6)}}=\left[\gamma_{y_{t}}^{(0)}\right]_{Q_{1,3 \cdots 8,3}^{(6)} \times Q_{1,3 \cdots 8,3}^{(6)}}=\left(\begin{array}{cccccccc}
1 & 0 & 0 & 0 & 0 & 0 & 0 & 0 \\
0 & 1 & -2 & 0 & 0 & 0 & 0 & 0 \\
0 & -1 & 2 & 0 & 0 & 0 & 0 & 0 \\
0 & 0 & 0 & 0 & 0 & 0 & 0 & 0 \\
0 & 0 & 0 & 0 & 1 & 0 & 0 & 0 \\
0 & 0 & 0 & 0 & 0 & 1 & -2 & 0 \\
0 & 0 & 0 & 0 & 0 & -1 & 2 & 0 \\
0 & 0 & 0 & 0 & 0 & 0 & 0 & 0
\end{array}\right)
$$

and

$$
\left[\gamma_{y_{b}}^{(0)}\right]_{Q_{1,3 \cdots 8,3}^{(6)} \times Q_{1,3 \cdots 8,3}^{(6)}}=\left(\begin{array}{cccccccc}
1 & 0 & 0 & 0 & 0 & 0 & 0 & 0 \\
0 & 1 & 0 & -2 & 0 & 0 & 0 & 0 \\
0 & 0 & 0 & 0 & 0 & 0 & 0 & 0 \\
0 & -1 & 0 & 2 & 0 & 0 & 0 & 0 \\
0 & 0 & 0 & 0 & 1 & 0 & 0 & 0 \\
0 & 0 & 0 & 0 & 0 & 1 & 0 & -2 \\
0 & 0 & 0 & 0 & 0 & 0 & 0 & 0 \\
0 & 0 & 0 & 0 & 0 & -1 & 0 & 2
\end{array}\right)
$$


The off-diagonal entries in eq. (3.38) are generated by the left-most diagram in figure 10 while the diagonal entries result from the field renormalization constants. The Yukawa interactions also lead to mixing of the DM-third-generation quark operators into the DMHiggs operators, $Q_{15}^{(6)}, \ldots, Q_{18}^{(6)}$,

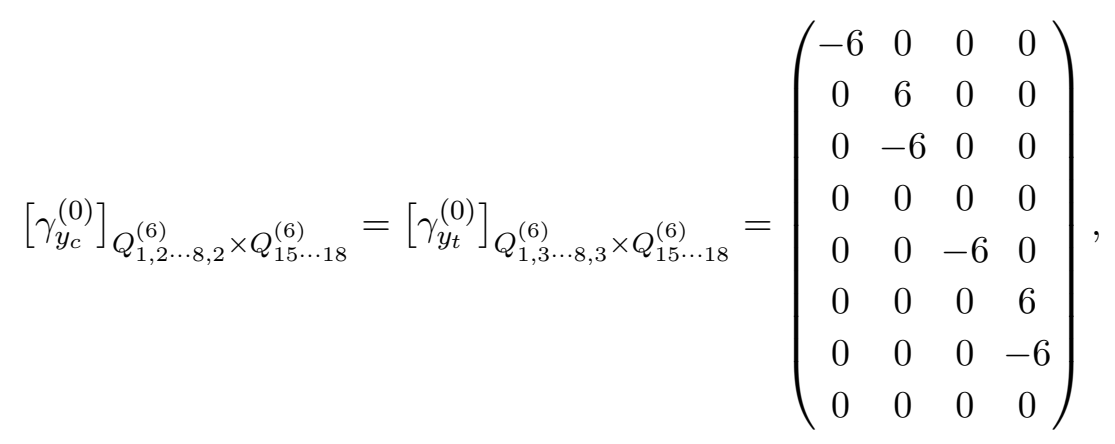

and

$$
\left[\gamma_{y_{b}}^{(0)}\right]_{Q_{1,3 \cdots 8,3}^{(6)} \times Q_{15 \cdots 18}^{(6)}}=\left(\begin{array}{cccc}
-6 & 0 & 0 & 0 \\
0 & -6 & 0 & 0 \\
0 & 0 & 0 & 0 \\
0 & 6 & 0 & 0 \\
0 & 0 & -6 & 0 \\
0 & 0 & 0 & -6 \\
0 & 0 & 0 & 0 \\
0 & 0 & 0 & 6
\end{array}\right)
$$

as well as to the mixing of the DM-Higgs operators into the DM-third-generation quark operators,

$$
\begin{aligned}
{\left[\gamma_{y_{c}}^{(0)}\right]_{Q_{15 \cdots 18}^{(6)} \times Q_{1,2 \cdots 8,2}^{(6)}}=\left[\gamma_{y_{t}}^{(0)}\right]_{Q_{15 \cdots 18}^{(6)} \times Q_{1,3 \cdots 8,3}^{(6)}}^{(6)}=\left(\begin{array}{cccccccc}
-1 & 0 & 0 & 0 & 0 & 0 & 0 & 0 \\
0 & 1 & -2 & 0 & 0 & 0 & 0 & 0 \\
0 & 0 & 0 & 0 & -1 & 0 & 0 & 0 \\
0 & 0 & 0 & 0 & 0 & 1 & -2 & 0
\end{array}\right) } \\
{\left[\gamma_{y_{b}}^{(0)}\right]_{Q_{15 \cdots 18}^{(6)} \times Q_{1,3 \cdots 8,3}^{(6)}}=\left(\begin{array}{cccccccc}
-1 & 0 & 0 & 0 & 0 & 0 & 0 & 0 \\
0 & -1 & 0 & 2 & 0 & 0 & 0 & 0 \\
0 & 0 & 0 & 0 & -1 & 0 & 0 & 0 \\
0 & 0 & 0 & 0 & 0 & -1 & 0 & 2
\end{array}\right) . }
\end{aligned}
$$

The Yukawa interactions also renormalize the Higgs operators themselves due to the renormalization of the Higgs fields, giving

$$
\left[\gamma_{y_{t}}^{(0)}\right]_{Q_{15 \cdots 18}^{(6)} \times Q_{15 \cdots 18}^{(6)}}=\left[\gamma_{y_{b}}^{(0)}\right]_{Q_{15 \cdots 18}^{(6)} \times Q_{15 \cdots 18}^{(6)}}=\left[\gamma_{y_{c}}^{(0)}\right]_{Q_{15 \cdots 18}^{(6)} \times Q_{15 \cdots 18}^{(6)}}=\operatorname{diag}(6,6,6,6)
$$

Finally, we also present the anomalous dimensions due to the tau Yukawa coupling, leading to mixing among the four-fermion operators,

$$
\left[\gamma_{y_{\tau}}^{(0)}\right]_{Q_{9,3 \cdots 14,3}^{(6)} \times Q_{9,3 \cdots 14,3}^{(6)}}=\left(\begin{array}{cccccc}
1 & 0 & 0 & 0 & 0 & 0 \\
0 & 1 & -2 & 0 & 0 & 0 \\
0 & -1 & 2 & 0 & 0 & 0 \\
0 & 0 & 0 & 1 & 0 & 0 \\
0 & 0 & 0 & 0 & 1 & -2 \\
0 & 0 & 0 & 0 & -1 & 2
\end{array}\right)
$$



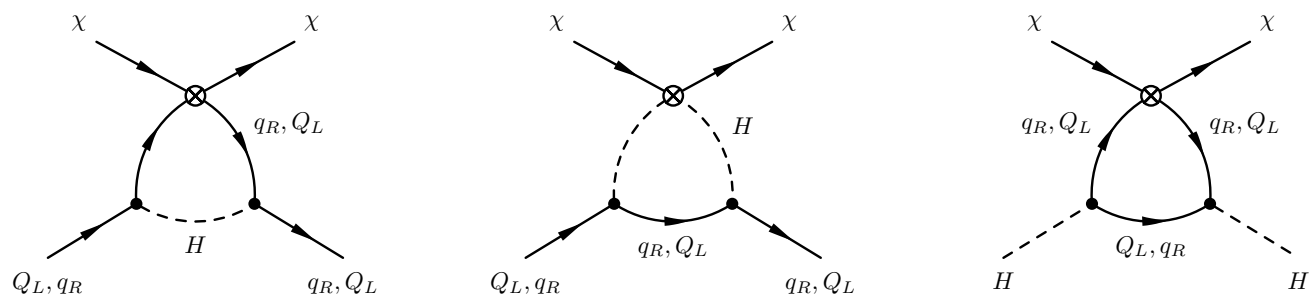

Figure 10. Mixing of the quark operators proportional to the quark yukawa coupling due to the insertion of DM-quark and DM-Higgs operators. We consider only the top, bottom, charm Yukawas here and so $q \in\{t, b, c\}$. The same diagrams with $Q_{L} \rightarrow L_{L}$ and $q_{R} \rightarrow \tau_{R}$ mix DM-lepton and DM-Higgs operators. These diagrams only contribute to off-diagonal mixing.

mixing of four-fermion into Higgs operators,

$$
\left[\gamma_{y_{\tau}}^{(0)}\right]_{Q_{9,3 \cdots 14,3}^{(6)} \times Q_{15 \cdots 18}^{(6)}}=\left(\begin{array}{cccc}
-2 & 0 & 0 & 0 \\
0 & -2 & 0 & 0 \\
0 & 2 & 0 & 0 \\
0 & 0 & -2 & 0 \\
0 & 0 & 0 & -2 \\
0 & 0 & 0 & 2
\end{array}\right)
$$

Higgs operators into four-fermion operators,

$$
\left[\gamma_{y_{\tau}}^{(0)}\right]_{Q_{15 \cdots 18}^{(6)} \times Q_{9,3 \cdots 14,3}^{(6)}}=\left(\begin{array}{cccccc}
-1 & 0 & 0 & 0 & 0 & 0 \\
0 & -1 & 2 & 0 & 0 & 0 \\
0 & 0 & 0 & -1 & 0 & 0 \\
0 & 0 & 0 & 0 & -1 & 2
\end{array}\right)
$$

and diagonal self mixing of the Higgs operators due to Higgs field renormalization,

$$
\left[\gamma_{y_{\tau}}^{(0)}\right]_{Q_{15 \cdots 18}^{(6)} \times Q_{15 \cdots 18}^{(6)}}=\operatorname{diag}(2,2,2,2) .
$$

The results given in this section are sufficient if one works to leading-log accuracy without resummation of the logarithms. However, the set of operators $Q_{1, i}^{(6)}, \ldots, Q_{18}^{(6)}$, eqs. (2.9)-(2.17), does not close under renormalization, unless the DM self-interaction operators, eq. (2.18), and the SM EFT operators are included. We provide the anomalous dimension that give the mixing with the SM effective operators in appendix C, and with the DM self-interaction operators in appendix D.

\section{Matching to EFT below the weak scale}

The running from the mediator scale, $\mu \sim \Lambda$, down to the weak scale, $\mu \sim m_{Z}$, is described by the evolution operator $U\left(\mu_{\mathrm{EW}}, \Lambda\right)$ in eq. (3.6). The relevant anomalous dimension matrix $\gamma$, appearing in eq. (3.4), was presented in sections 3.1 and 3.2. The next step is to calculate the matching onto a five-flavor theory at $\mu \sim v_{\mathrm{EW}}$ by integrating out the top quark, the Higgs and $W, Z$ gauge bosons. This gives the matrix $M_{\mathrm{EW} \rightarrow(5)}$ in the evolution equation (3.6). Since we are interested in the elastic direct detection scattering we can, 
below the electroweak scale, ignore all the charged components of the $\chi$ multiplet. From now on $\chi$ will thus denote only the neutral component of the DM electroweak multiplet.

After the matching at $\mu \sim m_{Z}$ we arrive at the $n_{f}=5$ effective Lagrangian which we organize in terms of the dimensionality of the operators,

$$
\left.\mathcal{L}_{\chi}\right|_{n_{f}=5}=\left.\mathcal{L}_{\chi}^{(4)}\right|_{n_{f}=5}+\left.\mathcal{L}_{\chi}^{(5)}\right|_{n_{f}=5}+\left.\mathcal{L}_{\chi}^{(6)}\right|_{n_{f}=5}+\left.\mathcal{L}_{\chi}^{(7)}\right|_{n_{f}=5}+\cdots
$$

In the matching we keep all the numerically leading terms. The leading contributions from dimension-five $(\propto 1 / \Lambda)$ and dimension-six operators $\left(\propto 1 / \Lambda^{2}\right)$, eq. (2.2), generically arise already at tree level, with the exception of phenomenologically important one-loop matchings onto the dimension-seven gluonic operators. In these matching calculations we allow for DM to carry arbitrary $\mathrm{SU}(2) \times \mathrm{U}(1)$ gauge quantum numbers. In addition, there are contributions from renormalizable interactions. We include these in our numerical examples in section 5, taking $Y_{\chi}=0$, so that there is no tree-level $Z$ coupling to DM. The first nonzero contributions from gauge interactions are then due to the one- and two-loop electroweak threshold corrections, shown in figure 2, for which we use the results of ref. [68].

We consider two discrete options for the DM mass: i) light DM, $m_{\chi} \ll m_{Z}$, and ii) DM with the EW scale mass, $m_{\chi} \sim \mathcal{O}\left(m_{Z}\right)$. The case of heavy DM, $m_{\chi} \gg m_{Z}$, is relegated to future work (dimension-four interactions are discussed in refs. [7, 81]). In section 4.1 we perform the matching for light DM. In this case the time component and the spatial components of the DM current are of the same size at the matching scale. The situation is different for weak scale DM. For $\mu \lesssim m_{\chi} \sim \mathcal{O}\left(m_{Z}\right)$ DM becomes nonrelativistic, and thus the time component is parametrically larger than the spatial ones. In the matching we therefore need to simultaneously perform an expansion in $1 / m_{\chi}$, which is done in section 4.2 .

Before proceeding we remark that both the DM mass, $m_{\chi}$, and the DM field, $\chi$, get shifted by the Higgs vacuum expectation value due to the contributions from the $Q_{3,4}^{(5)}$ operators, eq. (2.6), and from the $Q_{7,8}^{(5)}$ operators, eq. (2.8). The dimension-four part of the effective Lagrangian (4.1) in terms of the shifted fields, $\chi^{\prime}$, is

$$
\left.\mathcal{L}_{\chi}^{(4)}\right|_{n_{f}=5}=i \bar{\chi}^{\prime} \not \partial \chi^{\prime}-m_{\chi}^{\prime} \bar{\chi}^{\prime} \chi^{\prime}
$$

The redefinition of the $\chi$ field is a simple chiral rotation, $\chi^{\prime}=\exp \left(\frac{i}{2} \gamma_{5} \phi\right) \chi$, with (see also ref. [82])

$$
\tan \phi=\left(C_{7}^{(5)}+\frac{Y_{\chi}}{4} C_{8}^{(5)}\right) /\left[2 \pi \alpha_{2} m_{\chi} \Lambda /\left(c_{w}^{2} m_{Z}^{2}\right)-\left(C_{3}^{(5)}+\frac{Y_{\chi}}{4} C_{4}^{(5)}\right)\right]
$$

while the new mass term is

$$
m_{\chi}^{\prime}=m_{\chi} \cos \phi+\frac{c_{w}^{2} m_{Z}^{2}}{2 \pi \alpha_{2} \Lambda}\left[\left(C_{7}^{(5)}+\frac{Y_{\chi}}{4} C_{8}^{(5)}\right) \sin \phi-\left(C_{3}^{(5)}+\frac{Y_{\chi}}{4} C_{4}^{(5)}\right) \cos \phi\right] .
$$

The field redefinition also changes the operators $Q_{1}^{(5)}, \ldots, Q_{8}^{(5)}$ in eqs. (2.5)-(2.8) and the corresponding Wilson coefficients, $C_{i}^{(5) \prime}=C_{i}^{(5)} \cos \phi+C_{i+4}^{(5)} \sin \phi, C_{i+4}^{(5)}{ }^{\prime}=C_{i+4}^{(5)} \cos \phi-$ 
$C_{i}^{(5)} \sin \phi$, for $i=1, \ldots, 4$, while there is no change in the dimension-six Wilson coefficients. In the case $m_{\chi} \sim \mathcal{O}\left(m_{Z}\right)$ we expand in $m_{Z} / \Lambda$ which gives

$$
\begin{aligned}
C_{i}^{(5) \prime} & =C_{i}^{(5)}+\frac{c_{w}^{2} m_{Z}^{2}}{2 \pi \alpha_{2} \Lambda m_{\chi}}\left(C_{7}^{(5)}+\frac{Y_{\chi}}{4} C_{8}^{(5)}\right) C_{i+4}^{(5)}, \\
C_{i+4}^{(5) \prime} & =C_{i+4}^{(5)}-\frac{c_{w}^{2} m_{Z}^{2}}{2 \pi \alpha_{2} \Lambda m_{\chi}}\left(C_{7}^{(5)}+\frac{Y_{\chi}}{4} C_{8}^{(5)}\right) C_{i}^{(5)} .
\end{aligned}
$$

From now on we will assume that the above field and mass redefinitions have been performed and drop the primes on the Wilson coefficients, the DM fields, and the DM mass.

\subsection{Light dark matter}

In the case of light DM, $m_{\chi} \ll m_{Z}$, we can use relativistic DM fields to construct the effective theory below the weak scale. The effective Lagrangians containing operators of dimensionality $d$ in eq. (4.1) are given by

$$
\left.\mathcal{L}_{\chi}^{(d)}\right|_{n_{f}=5}=\left.\sum_{a} \hat{\mathcal{C}}_{a}^{(d)}\right|_{n_{f}=5} \mathcal{Q}_{a}^{(d)},
$$

where we introduced the dimensionful Wilson coefficients $\left.\hat{\mathcal{C}}_{a}^{(d)}\right|_{n_{f}=5}$ in order to simplify the notation. They are suppressed by inverse powers of the NP scale $\Lambda$ and/or the top, $W$, $Z$ and Higgs masses. The DM mass, $m_{\chi}$, can be set to zero in the matching except when calculating the electroweak threshold corrections from the gauge interactions, where one needs to expand to first order in $m_{\chi}$.

The electroweak EFT Lagrangian (2.2) with operators up to dimension six matches onto the "five-flavor" EFT in the broken electroweak phase, eq. (4.1). This gives rise to operators up to dimension seven, if one keeps only the leading contributions. We first give the basis of the operators $\mathcal{Q}_{a}^{(d)}$ in the five-flavor EFT, required for the matching, and then present their respective Wilson coefficients $\hat{\mathcal{C}}_{a}^{(d)}$.

At dimension five there are only two operators,

$$
\mathcal{Q}_{1}^{(5)}=\frac{e}{8 \pi^{2}}\left(\bar{\chi} \sigma^{\mu \nu} \chi\right) F_{\mu \nu}, \quad \mathcal{Q}_{2}^{(5)}=\frac{e}{8 \pi^{2}}\left(\bar{\chi} \sigma^{\mu \nu} i \gamma_{5} \chi\right) F_{\mu \nu}
$$

where $F_{\mu \nu}$ is the electromagnetic field strength tensor. The operator $\mathcal{Q}_{1}^{(5)}$ is CP even, while $\mathcal{Q}_{2}^{(5)}$ is CP odd. The dimension-six operators are

$$
\begin{array}{llrl}
\mathcal{Q}_{1, f}^{(6)} & =\left(\bar{\chi} \gamma_{\mu} \chi\right)\left(\bar{f} \gamma^{\mu} f\right), & \mathcal{Q}_{2, f}^{(6)}=\left(\bar{\chi} \gamma_{\mu} \gamma_{5} \chi\right)\left(\bar{f} \gamma^{\mu} f\right), \\
\mathcal{Q}_{3, f}^{(6)}=\left(\bar{\chi} \gamma_{\mu} \chi\right)\left(\bar{f} \gamma^{\mu} \gamma_{5} f\right), & \mathcal{Q}_{4, f}^{(6)}=\left(\bar{\chi} \gamma_{\mu} \gamma_{5} \chi\right)\left(\bar{f} \gamma^{\mu} \gamma_{5} f\right) .
\end{array}
$$

Here $f$ denotes any quark, $f=u, d, s, c, b$, or charged lepton flavor, $f=e, \mu, \tau$. We find it convenient to express the operators in terms of (axial-)vector and (pseudo-)scalar currents, which have definite non-relativistic limits. Operators with neutrinos are not needed for our purposes as they do not run below the EW scale.

In the effective Lagrangian eq. (4.1) we need to include a subset of dimension-seven operators. These are generated from dimension-five and -six operators in the effective 
Lagrangian (2.2) when integrating out the Higgs and the $Z$ boson at $\mu_{\mathrm{EW}} \sim m_{Z}$. They are thus suppressed by $\mathcal{O}\left(1 / \Lambda^{2} m_{h, Z}\right)$ or $\mathcal{O}\left(1 / \Lambda m_{h, Z}^{2}\right)$, instead of $\mathcal{O}\left(1 / \Lambda^{3}\right)$, and can lead to contributions in direct detection comparable to those of the dimension-six operators, eqs. (4.8)-(4.9).

The relevant dimension-seven operators involving the DM and gluon fields are given by

$$
\begin{array}{rlrl}
\mathcal{Q}_{1}^{(7)} & =\frac{\alpha_{s}}{12 \pi}(\bar{\chi} \chi) G^{a \mu \nu} G_{\mu \nu}^{a}, & & \mathcal{Q}_{2}^{(7)}=\frac{\alpha_{s}}{12 \pi}\left(\bar{\chi} i \gamma_{5} \chi\right) G^{a \mu \nu} G_{\mu \nu}^{a}, \\
\mathcal{Q}_{3}^{(7)}=\frac{\alpha_{s}}{8 \pi}(\bar{\chi} \chi) G^{a \mu \nu} \widetilde{G}_{\mu \nu}^{a}, & \mathcal{Q}_{4}^{(7)}=\frac{\alpha_{s}}{8 \pi}\left(\bar{\chi} i \gamma_{5} \chi\right) G^{a \mu \nu} \widetilde{G}_{\mu \nu}^{a},
\end{array}
$$

where $\widetilde{G}_{\mu \nu}=\frac{1}{2} \varepsilon_{\mu \nu \rho \sigma} G^{\rho \sigma}$ and $a=1, \ldots, 8$ are the color indices. The strong coupling constant $\alpha_{s}$ is defined in the five-flavor scheme. The normalization reflects the fact that these operators are typically generated at one-loop level. Note that $\mathcal{Q}_{2}^{(7)}$ and $\mathcal{Q}_{3}^{(7)}$ are CP odd.

There are also four scalar operators

$$
\begin{aligned}
\mathcal{Q}_{5, f}^{(7)} & =m_{f}(\bar{\chi} \chi)(\bar{f} f), & \mathcal{Q}_{6, f}^{(7)} & =m_{f}\left(\bar{\chi} i \gamma_{5} \chi\right)(\bar{f} f), \\
\mathcal{Q}_{7, f}^{(7)} & =m_{f}(\bar{\chi} \chi)\left(\bar{f} i \gamma_{5} f\right), & \mathcal{Q}_{8, f}^{(7)} & =m_{f}\left(\bar{\chi} i \gamma_{5} \chi\right)\left(\bar{f} i \gamma_{5} f\right),
\end{aligned}
$$

with $f$ denoting any quark $(f=u, d, s, c, b)$ or charged lepton flavor $(f=e, \mu, \tau)$. The definitions of $\mathcal{Q}_{5, f}^{(7)}, \ldots, \mathcal{Q}_{8, f}^{(7)}$ include an explicit power of the corresponding quark or lepton mass. This reflects the leading contributions to their Wilson coefficients, see below.

In the remainder of the subsection we give the results of the matching at $\mu_{\mathrm{EW}} \sim$ $m_{Z}$. We start with the dimension-five operators where the contributions come from $W$ and $B$ dipole operators above $m_{Z}$ after rotating the EW gauge eigenstates into the mass eigenstates after EWSB: ${ }^{4}$

$$
\begin{aligned}
& \left.\hat{\mathcal{C}}_{1}^{(5)}\right|_{n_{f}=5}=\frac{1}{\Lambda}\left(C_{1}^{(5)}+\frac{Y_{\chi}}{2} C_{2}^{(5)}\right)+\ldots, \\
& \left.\hat{\mathcal{C}}_{2}^{(5)}\right|_{n_{f}=5}=\frac{1}{\Lambda}\left(C_{5}^{(5)}+\frac{Y_{\chi}}{2} C_{6}^{(5)}\right) .
\end{aligned}
$$

Equation (4.14) also receives a one-loop contribution from dimension-four gauge interactions, denoted by the ellipsis, proportional to the hypercharge of the DM multiplet. We omit this contribution here since a non-zero hypercharge leads to a tree-level $Z$ exchange with nuclei which is excluded by direct detection experiments.

For the dimension-six operators we start with the operators with external quark legs. The contributions from dimension-six UV operators with external quark legs are

$$
\begin{aligned}
& \left.\hat{\mathcal{C}}_{1, u_{i}\left(d_{i}\right)}^{(6)}\right|_{n_{f}=5}=\frac{1}{\Lambda^{2}}\left[\mp \frac{Y_{\chi}}{8} C_{1, i}^{(6)}+\frac{C_{2, i}^{(6)}}{2}+\frac{C_{3(4), i}^{(6)}}{2} \pm \frac{3-8(4) s_{w}^{2}}{6}\left(\frac{Y_{\chi}}{4} C_{15}^{(6)}+C_{16}^{(6)}\right)\right], \\
& \left.\hat{\mathcal{C}}_{2, u_{i}\left(d_{i}\right)}^{(6)}\right|_{n_{f}=5}=\frac{1}{\Lambda^{2}}\left[\mp \frac{Y_{\chi}}{8} C_{5, i}^{(6)}+\frac{C_{6, i}^{(6)}}{2}+\frac{C_{7(8), i}^{(6)}}{2} \pm \frac{3-8(4) s_{w}^{2}}{6}\left(\frac{Y_{\chi}}{4} C_{17}^{(6)}+C_{18}^{(6)}\right)\right]
\end{aligned}
$$

\footnotetext{
${ }^{4}$ Note that in eqs. (4.14) and (4.15) we use the original definition of operators, eqs. (2.5) and (2.7).
} 

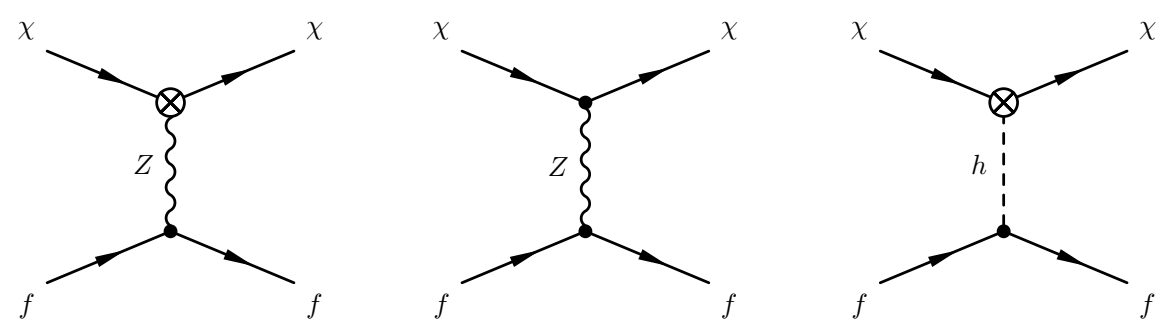

Figure 11. Matching contributions to the effective operators at $\mu \sim m_{Z}$. The left diagram shows the contribution of the high-energy operators, the middle one the contribution from the dimensionfour gauge interactions onto the dimension-six operators, respectively; the right diagram shows the contribution to the matching onto the dimension-seven operators.

$$
\begin{aligned}
& \left.\hat{\mathcal{C}}_{3, u_{i}\left(d_{i}\right)}^{(6)}\right|_{n_{f}=5}=\frac{1}{\Lambda^{2}}\left[ \pm \frac{Y_{\chi}}{8} C_{1, i}^{(6)}-\frac{C_{2, i}^{(6)}}{2}+\frac{C_{3(4), i}^{(6)}}{2} \mp \frac{1}{2}\left(\frac{Y_{\chi}}{4} C_{15}^{(6)}+C_{16}^{(6)}\right)\right] \\
& \left.\hat{\mathcal{C}}_{4, u_{i}\left(d_{i}\right)}^{(6)}\right|_{n_{f}=5}=\frac{1}{\Lambda^{2}}\left[ \pm \frac{Y_{\chi}}{8} C_{5, i}^{(6)}-\frac{C_{6, i}^{(6)}}{2}+\frac{C_{7(8), i}^{(6)}}{2} \mp \frac{1}{2}\left(\frac{Y_{\chi}}{4} C_{17}^{(6)}+C_{18}^{(6)}\right)\right]+\ldots,
\end{aligned}
$$

where $i$ is a generation index $\left(u_{1} \equiv u, u_{2} \equiv c\right.$ and $\left.d_{1} \equiv d, d_{2} \equiv s, d_{3} \equiv b\right)$ and the upper(lower) signs apply for up(down) quarks. For each of the Wilson coefficients the last $1 / \Lambda^{2}$-suppressed term is due to $Z$ exchange, shown in figure 11 (left). For a DM multiplet with nonzero hypercharge $Y_{\chi}, Z$ exchange due to the renormalizable gauge coupling (2.3), see figure 11 (right), gives the additional contributions $\left.\hat{\mathcal{C}}_{1, u_{i}\left(d_{i}\right)}^{(6)}\right|_{n_{f}=5}=$ $\pm \frac{\pi \alpha_{2}}{6 c_{w}^{2} m_{Z}^{2}}\left(3-8(4) s_{w}^{2}\right) Y_{\chi}$ and $\left.\hat{\mathcal{C}}_{3, u_{i}\left(d_{i}\right)}^{(6)}\right|_{n_{f}=5}=\left.\mp \frac{\pi \alpha_{2}}{2 c_{w}^{2} m_{Z}^{2}} Y_{\chi} \cdot \hat{\mathcal{C}}_{4, u_{i}\left(d_{i}\right)}^{(6)}\right|_{n_{f}=5}$ receives a contribution, denoted by the ellipsis, from gauge interactions at one-loop (see figure 2) that does not vanish for $Y_{\chi}=0$. This requires a two-loop matching calculation with $m_{\chi}$ kept parametrically small, which is beyond the scope of present paper. In the numerical evaluations we thus use the results from ref. [68], that were obtained assuming that $m_{\chi}$ is not much smaller than $m_{Z}$.

Similarly we find for the dimension-six operators with leptons $\left(\ell_{1} \equiv e, \ell_{2} \equiv \mu, \ell_{3} \equiv \tau\right)$ on the external legs

$$
\begin{aligned}
& \left.\hat{\mathcal{C}}_{1, \ell_{i}}^{(6)}\right|_{n_{f}=5}=\frac{1}{\Lambda^{2}}\left[\frac{Y_{\chi}}{8} C_{9, i}^{(6)}+\frac{C_{10, i}^{(6)}}{2}+\frac{C_{11, i}^{(6)}}{2}-\frac{1-4 s_{w}^{2}}{2}\left(\frac{Y_{\chi}}{4} C_{15}^{(6)}+C_{16}^{(6)}\right)\right], \\
& \left.\hat{\mathcal{C}}_{2, \ell_{i}}^{(6)}\right|_{n_{f}=5}=\frac{1}{\Lambda^{2}}\left[\frac{Y_{\chi}}{8} C_{12, i}^{(6)}+\frac{C_{13, i}^{(6)}}{2}+\frac{C_{14, i}^{(6)}}{2}-\frac{1-4 s_{w}^{2}}{2}\left(\frac{Y_{\chi}}{4} C_{17}^{(6)}+C_{18}^{(6)}\right)\right], \\
& \left.\hat{\mathcal{C}}_{3, \ell_{i}}^{(6)}\right|_{n_{f}=5}=\frac{1}{\Lambda^{2}}\left[-\frac{Y_{\chi}}{8} C_{9, i}^{(6)}-\frac{C_{10, i}^{(6)}}{2}+\frac{C_{11, i}^{(6)}}{2}+\frac{1}{2}\left(\frac{Y_{\chi}}{4} C_{15}^{(6)}+C_{16}^{(6)}\right)\right], \\
& \left.\hat{\mathcal{C}}_{4, \ell_{i}}^{(6)}\right|_{n_{f}=5}=\frac{1}{\Lambda^{2}}\left[-\frac{Y_{\chi}}{8} C_{12, i}^{(6)}-\frac{C_{13, i}^{(6)}}{2}+\frac{C_{14, i}^{(6)}}{2}+\frac{1}{2}\left(\frac{Y_{\chi}}{4} C_{17}^{(6)}+C_{18}^{(6)}\right)\right]+\ldots
\end{aligned}
$$

As before, $Z$-boson exchange due to the renormalizable gauge coupling (2.3) leads to the additional contributions $\left.\hat{\mathcal{C}}_{1, \ell_{i}}^{(6)}\right|_{n_{f}=5}=-\frac{\pi \alpha_{2}}{2 c_{w}^{2} m_{Z}^{2}}\left(1-4 s_{w}^{2}\right) Y_{\chi}$ and $\left.\hat{\mathcal{C}}_{3, \ell_{i}}^{(6)}\right|_{n_{f}=5}=\frac{\pi \alpha_{2}}{2 c_{w}^{2} m_{Z}^{2}} Y_{\chi}$. Also 


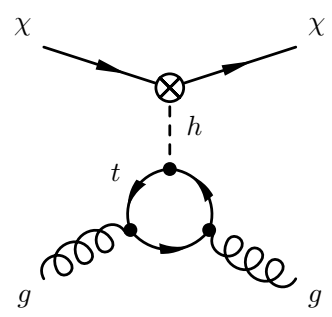

Figure 12. Matching contributions to dimension-seven effective operators involving gluons for $\mu<m_{Z}$ that arise from integrating out the top quark.

for leptons, $\left.\hat{\mathcal{C}}_{4, \ell_{i}}^{(6)}\right|_{n_{f}=5}$ receives a one-loop contribution from gauge interaction that does not vanish for $Y_{\chi}=0$, see ref. [68].

The dimension-seven operators receive contributions from both the renormalizable electroweak interactions of the DM multiplet as well as from the higher dimension operators. For the gluonic operators $\mathcal{Q}_{1,2}^{(7)}$ the higher dimension UV operators give a contribution after integrating out the top quark at one loop, see figure 12 . We then have

$$
\left.\hat{\mathcal{C}}_{1(2)}^{(7)}\right|_{n_{f}=5}=\frac{1}{\Lambda m_{h}^{2}}\left(C_{3(7)}^{(5)}+\frac{Y_{\chi}}{4} C_{4(8)}^{(5)}\right)+\ldots,
$$

and $\left.\hat{\mathcal{C}}_{3(4)}^{(7)}\right|_{n_{f}=5}=0$. Note that the loop factor is already included in the definition of the operators $\mathcal{Q}_{i}^{(7)}$. The explicit top-quark mass dependence drops out because we expand to leading (quadratic) order in the small external momenta. This limit is equivalent to the limit of heavy top mass in on-shell Higgs decays to two photons or gluons, where the non-decoupling of chiral fermions is a familiar result. The ellipsis denotes the two-loop contributions from renormalizable electroweak interactions, see ref. [68].

For scalar operators we have

$$
\begin{aligned}
& \left.\hat{\mathcal{C}}_{5, f}^{(7)}\right|_{n_{f}=5}=-\frac{1}{\Lambda m_{h}^{2}}\left(C_{3}^{(5)}+\frac{Y_{\chi}}{4} C_{4}^{(5)}\right)+\ldots, \\
& \left.\hat{\mathcal{C}}_{6, f}^{(7)}\right|_{n_{f}=5}=-\frac{1}{\Lambda m_{h}^{2}}\left(C_{7}^{(5)}+\frac{Y_{\chi}}{4} C_{8}^{(5)}\right),
\end{aligned}
$$

and $\left.\hat{\mathcal{C}}_{7, f}^{(7)}\right|_{n_{f}=5}=\left.\hat{\mathcal{C}}_{8, f}^{(7)}\right|_{n_{f}=5}=0$. The right diagram in figure 11 shows the tree-level contributions from higher dimension operators. Here, the ellipsis denotes the one-loop "Higgs penguin" contribution from gauge interactions (see figure 2 and ref. [68]).

\subsection{Electroweak scale dark matter}

The case that the DM mass is comparable to the electroweak scale, $m_{\chi} \sim \mathcal{O}\left(m_{Z}\right)$, needs to be treated separately. In this case we integrate out at the electroweak scale, in addition to the top quark, the Higgs and the $W, Z$ bosons, also the high-momentum fluctuations of the DM field. In this way we arrive at the Heavy Dark Matter Effective Theory (HDMET). The HDMET is an effective theory the describes the scattering of a heavy DM, where the momenta exchanges are much smaller than the DM mass, $q \ll m_{\chi}$. The HDMET uses the 
HQET (Heavy Quark Effective Theory) formalism [83-85] applied to DM direct detection physics [8]. The result is an effective theory where terms are organized as an expansion in $1 / m_{\chi}$. In practice the HDMET description is not necessary if one performs electroweak matching only at tree level. However, some of the one-loop matching corrections are important phenomenologically and need to be kept, requiring the use of HDMET.

The construction of the requisite terms in HDMET has been presented in ref. [20]. Here, we just collect the main results and refer the interested reader to the literature for details.

The HDMET Lagrangian is given by ${ }^{5}$

$$
\mathcal{L}_{\mathrm{HDMET}}=\bar{\chi}_{v}(i v \cdot \partial) \chi_{v}+\frac{1}{2 m_{\chi}} \bar{\chi}_{v}\left(i \partial_{\perp}\right)^{2} \chi_{v}+\ldots+\left.\sum_{d} \mathcal{L}_{\chi_{v}}^{(d)}\right|_{n_{f}=5}
$$

Here, $\chi_{v}$ denotes only the neutral component of the DM electroweak multiplet, i.e., only the DM state. The first term is the LO HDMET Lagrangian and describes an infinitely heavy DM particle, and contains no explicit dependence on $m_{\chi}$. The $\mathcal{O}\left(1 / m_{\chi}\right)$ term is fixed by reparametrization invariance [87], with ellipsis denoting terms of higher order in the $1 / m_{\chi}$ expansion.

The effective Lagrangians $\left.\mathcal{L}_{\chi v}^{(d)}\right|_{n_{f}=5}$ comprise the interactions of DM with the SM. They are expanded in powers of $1 / m_{\chi}, 1 / \Lambda$ and $1 / m_{Z}$, mirroring the case of light DM in eq. (4.6). The only difference is that we now denote explicitly at which order in $1 / m_{\chi}$ the operators enter,

$$
\left.\mathcal{L}_{\chi v}^{(d)}\right|_{n_{f}=5}=\left.\sum_{a, m} \hat{\mathcal{C}}_{a}^{(d, m)}\right|_{n_{f}=5} \mathcal{Q}_{a}^{(d, m)}
$$

such that $\left.\hat{\mathcal{C}}_{a}^{(d, m)}\right|_{n_{f}=5} \propto\left(\Lambda, m_{Z}\right)^{4+m-d} m_{\chi}^{-m}$, where $\left(\Lambda, m_{Z}\right)^{4+m-d}$ symbolizes a product of powers of $\Lambda$ and $m_{Z}$ with total power $4+m-d$. The double superscripts on $\hat{\mathcal{C}}_{a}^{(d, m)}$ and $\mathcal{Q}_{a}^{(d, m)}$ thus signal that they are defined in the HDMET, while a single superscript on $\hat{\mathcal{C}}_{a}^{(d)}$ or on $\mathcal{Q}_{a}^{(d)}$ means that we are considering light DM.

The difference $d-m$ gives the "mediator dimensionality". This is the dimension of the relativistic operator $\mathcal{Q}_{a}^{(d-m)}$ that gives the HDMET operator $\mathcal{Q}_{a}^{(d, m)}$ upon expanding the DM currents to order $1 / m_{\chi}^{m}$ (see refs. [20, 21] for the explicit expressions). ${ }^{6}$ We group the operators in terms of their mediator dimensionality, $d-m$. The operators that arise at LO

${ }^{5}$ For very heavy DM, $m_{\chi} \gg m_{Z}$, the DM mass is integrated out before the weak gauge bosons $[8,16$, $81,86]$, giving

$$
\mathcal{L}_{\mathrm{HDMET}}=\bar{\chi}_{v}(i v \cdot D) \chi_{v}+\frac{1}{2 m_{\chi}} \bar{\chi}_{v}\left(i D_{\perp}\right)^{2} \chi_{v}+\frac{g_{2} c_{W}}{4 m_{\chi}} \bar{\chi}_{v} \sigma_{\mu \nu} \tilde{\tau} \cdot W^{\mu \nu} \chi_{v}+\frac{g_{1} c_{B}}{4 m_{\chi}} \bar{\chi}_{v} \sigma_{\mu \nu} B^{\mu \nu} \chi_{v}+\cdots
$$

where at tree level $c_{W}=c_{B}=1$, and the ellipsis denotes terms of higher order in $1 / m_{\chi}$, as well as the $1 / \Lambda$ suppressed interactions. The covariant derivative contains the $W_{\mu}^{a}$ and $B_{\mu}$ gauge fields, so that in the infinite mass limit the DM multiplet, $\chi_{v}$, acts as a static source of the electroweak gauge fields.

${ }^{6}$ Note that the $\Lambda \gg m_{Z} \sim m_{\chi}$ limit reduces the set of HDMET operators that are generated. For instance, at dimension seven the operator $\left(\bar{\chi}_{v} \chi_{v}\right) G^{a \mu \nu} G_{\mu \nu}^{a}$ arises in the matching, but not the operator $\left(\bar{\chi}_{v} \chi_{v}\right) v^{\mu} v^{\nu} G_{\mu \rho}^{a} G_{\nu}^{a \rho}$. The latter would arise from the dimension-nine UV operator $\left(\bar{\chi} \partial^{\mu} \partial^{\nu} \chi\right) G_{\mu \rho}^{a} G_{\nu}^{a \rho}$ and is thus $m_{\chi}^{2} / \Lambda^{2}$ suppressed. In contrast, for $m_{\chi} \sim \Lambda$ the two operators are of the same size, and thus both arise in the matching to HDMET at scale $\mu \sim \Lambda$ (see, e.g., the discussion of twist-two operators in ref. [88]). 
in $1 / m_{\chi}$, i.e., for which the Wilson coefficients start at order $\mathcal{O}\left(1 / m_{\chi}^{0}\right)$, are the HDMET counterparts of the operators in eqs. (4.7)-(4.13). The two dimension-five operators in eq. (4.7) get replaced by the HDMET operators

$$
\mathcal{Q}_{1}^{(5,0)}=\frac{e}{4 \pi^{2}} \epsilon_{\mu \nu \alpha \beta}\left(\bar{\chi}_{v} S_{\chi}^{\alpha} v^{\beta} \chi_{v}\right) F^{\mu \nu}, \quad \mathcal{Q}_{2}^{(5,0)}=\frac{e}{2 \pi^{2}}\left(\bar{\chi}_{v} S_{\chi}^{\mu} v^{\nu} \chi_{v}\right) F_{\mu \nu} .
$$

We also need the following two subleading operators

$$
\mathcal{Q}_{1}^{(6,1)}=\frac{i e}{8 \pi^{2}}\left(\bar{\chi}_{v} v^{\mu} \sigma_{\perp}^{\nu \rho} \stackrel{\leftrightarrow}{\partial}_{\rho} \chi_{v}\right) F_{\mu \nu}, \quad \mathcal{Q}_{2}^{(6,1)}=-\frac{e}{8 \pi^{2}}\left(v^{\mu} \partial^{\nu} \bar{\chi}_{v} \chi_{v}\right) F_{\mu \nu},
$$

since the presence of the photon pole in the interaction of the magnetic dipole with the nuclear current requires that we go to the second order in the expansion of the DM tensor current. We defined $\sigma_{\perp}^{\mu \nu}=i\left[\gamma_{\perp}^{\mu}, \gamma_{\perp}^{\nu}\right] / 2, \gamma_{\perp}^{\mu}=\gamma^{\mu}-v^{\mu} \psi, \bar{\chi}_{v} \stackrel{\leftrightarrow}{\partial^{\mu}} \chi_{v}=\bar{\chi}_{v}\left(\partial^{\mu} \chi_{v}\right)-\left(\partial^{\mu} \bar{\chi}_{v}\right) \chi_{v}$ and $S^{\mu}=\gamma_{\perp}^{\mu} \gamma_{5} / 2$ is the spin operator, while $v^{\mu}=(1, \overrightarrow{0})$ is the velocity label of the nonrelativistic DM field (cf. ref. [20]).

At tree-level we have

$$
\begin{array}{ll}
\left.\left.\hat{\mathcal{C}}_{1}^{(5,0)}\right|_{n_{f}=5} \stackrel{\text { tree }}{=} \hat{\mathcal{C}}_{1}^{(5)}\right|_{n_{f}=5}+\cdots, & \left.\left.\hat{\mathcal{C}}_{2}^{(5,0)}\right|_{n_{f}=5} \stackrel{\text { tree }}{=} \hat{\mathcal{C}}_{2}^{(5)}\right|_{n_{f}=5}, \\
\left.\left.\hat{\mathcal{C}}_{1}^{(6,1)}\right|_{n_{f}=5} \stackrel{\text { tree }}{=} \frac{1}{m_{\chi}} \hat{\mathcal{C}}_{1}^{(5,0)}\right|_{n_{f}=5}, & \left.\left.\hat{\mathcal{C}}_{2}^{(6,1)}\right|_{n_{f}=5} \stackrel{\text { tree }}{=} \frac{1}{m_{\chi}} \hat{\mathcal{C}}_{1}^{(5,0)}\right|_{n_{f}=5},
\end{array}
$$

where the equalities get corrections at loop level. Again, $\left.\hat{\mathcal{C}}_{1}^{(5,0)}\right|_{n_{f}=5}$ receives a photon penguin contribution proportional to $Y_{\chi}$, denoted by the ellipsis and omitted in the following. The Wilson coefficients for the dipole operator in the case of light DM, $\hat{\mathcal{C}}_{1,2}^{(5)}$, are given in eqs. (4.14), (4.15).

The dimension-six operators of LO in $1 / m_{\chi}$ are

$$
\begin{array}{ll}
\mathcal{Q}_{1, f}^{(6,0)}=\left(\bar{\chi}_{v} \chi_{v}\right)(\bar{f} \psi f), & \mathcal{Q}_{2, f}^{(6,0)}=2\left(\bar{\chi}_{v} S_{\chi, \mu} \chi_{v}\right)\left(\bar{f} \gamma^{\mu} f\right), \\
\mathcal{Q}_{3, f}^{(6,0)}=\left(\bar{\chi}_{v} \chi_{v}\right)\left(\bar{f} \psi \gamma_{5} f\right), & \mathcal{Q}_{4, f}^{(6,0)}=2\left(\bar{\chi}_{v} S_{\chi, \mu} \chi_{v}\right)\left(\bar{f} \gamma^{\mu} \gamma_{5} f\right) .
\end{array}
$$

In addition, we need the following $d-m=6$ operators that are $1 / m_{\chi}$ suppressed

$$
\begin{array}{rlrl}
\mathcal{Q}_{1, f}^{(7,1)} & =\frac{1}{2}\left(\bar{\chi}_{v} i \stackrel{\leftrightarrow}{\partial}_{\perp}^{\mu} \chi_{v}\right)\left(\bar{f} \gamma_{\mu} f\right), & & \mathcal{Q}_{2, f}^{(7,1)}=-i\left(\bar{\chi}_{v} S_{\chi} \cdot \stackrel{\leftrightarrow}{\partial} \chi_{v}\right)(\bar{f} \psi f), \\
\mathcal{Q}_{3, f}^{(7,1)}=\frac{1}{2}\left(\bar{\chi}_{v} i \stackrel{\leftrightarrow}{\partial}{ }_{\perp}^{\mu} \chi_{v}\right)\left(\bar{f} \gamma_{\mu} \gamma_{5} f\right), & \mathcal{Q}_{4, f}^{(7,1)}=-i\left(\bar{\chi}_{v} S_{\chi} \cdot \stackrel{\leftrightarrow}{\partial} \chi_{v}\right)\left(\bar{f} \psi \gamma_{5} f\right), \\
\mathcal{Q}_{5, f}^{(7,1)}=\frac{1}{2} \partial_{\nu}\left(\bar{\chi}_{v} \sigma_{\perp}^{\mu \nu} \chi_{v}\right)\left(\bar{f} \gamma_{\mu} f\right), & \mathcal{Q}_{6, f}^{(7,1)}=\frac{1}{2} \partial_{\nu}\left(\bar{\chi}_{v} \sigma_{\perp}^{\mu \nu} \chi_{v}\right)\left(\bar{f} \gamma_{\mu} \gamma_{5} f\right),
\end{array}
$$

where our convention is that the derivatives act only within the brackets or on the nearest bracket. The $\mathcal{Q}_{1, f}^{(7,1)}, \mathcal{Q}_{2, f}^{(7,1)}$ operators do not enter the phenomenological analysis, but we keep them for completeness and transparency of notation. For the matching conditions we have

$$
\left.\hat{\mathcal{C}}_{i, f}^{(6,0)}\right|_{n_{f}=5}=\left.\left.m_{\chi} \hat{\mathcal{C}}_{i, f}^{(7,1)}\right|_{n_{f}=5} \stackrel{\text { tree }}{=} \hat{\mathcal{C}}_{i, f}^{(6)}\right|_{n_{f}=5}, \quad i=1, \ldots, 4 ;
$$

and in addition

$$
\left.\left.\mathcal{C}_{5, f}^{(7,1)}\right|_{n_{f}=5} \stackrel{\text { tree }}{=} \frac{1}{m_{\chi}} \hat{\mathcal{C}}_{1, f}^{(6)}\right|_{n_{f}=5},\left.\left.\quad \mathcal{C}_{6, f}^{(7,1)}\right|_{n_{f}=5} \stackrel{\text { tree }}{=} \frac{1}{m_{\chi}} \hat{\mathcal{C}}_{3, f}^{(6)}\right|_{n_{f}=5}
$$


Note that the equalities denoted by "tree" are only valid for tree-level matching, while the remaining relations are valid to all orders due to reparametrization invariance. The light DM Wilson coefficients $C_{i, f}^{(6)}$ are given in eqs. (4.16)-(4.23) .

The relevant dimension-seven operators in eqs. (4.10)-(4.13) involve scalar and pseudoscalar DM currents. The HDMET scalar current operator starts at $\mathcal{O}\left(1 / m_{\chi}^{0}\right)$, while pseudoscalar current starts at $\mathcal{O}\left(1 / m_{\chi}\right)$, see ref. [20]. We thus define the following $d-m=7$ HDMET operators

$$
\begin{array}{lll}
\mathcal{Q}_{1}^{(7,0)}=\frac{\alpha_{s}}{12 \pi}\left(\bar{\chi}_{v} \chi_{v}\right) G^{a \mu \nu} G_{\mu \nu}^{a}, & \mathcal{Q}_{2}^{(8,1)}=\frac{\alpha_{s}}{12 \pi} \partial_{\mu}\left(\bar{\chi}_{v} S_{\chi}^{\mu} \chi_{v}\right) G^{a \mu \nu} G_{\mu \nu}^{a}, \\
\mathcal{Q}_{3}^{(7,0)}=\frac{\alpha_{s}}{8 \pi}\left(\bar{\chi}_{v} \chi_{v}\right) G^{a \mu \nu} \widetilde{G}_{\mu \nu}^{a}, & \mathcal{Q}_{4}^{(8,1)}=\frac{\alpha_{s}}{8 \pi} \partial_{\mu}\left(\bar{\chi}_{v} S_{\chi}^{\mu} \chi_{v}\right) G^{a \mu \nu} \widetilde{G}_{\mu \nu}^{a}, \\
\mathcal{Q}_{5, f}^{(7,0)}=m_{f}\left(\bar{\chi}_{v} \chi_{v}\right)(\bar{f} f), & \mathcal{Q}_{6, f}^{(8,1)}=m_{f} \partial_{\mu}\left(\bar{\chi}_{v} S_{\chi}^{\mu} \chi_{v}\right)(\bar{f} f), \\
\mathcal{Q}_{7, f}^{(7,0)}=m_{f}\left(\bar{\chi}_{v} \chi_{v}\right)\left(\bar{f} i \gamma_{5} f\right), & \mathcal{Q}_{8, f}^{(8,1)}=-m_{f} \partial_{\mu}\left(\bar{\chi}_{v} S_{\chi}^{\mu} \chi_{v}\right)\left(\bar{f} i \gamma_{5} f\right) .
\end{array}
$$

The top-quark loop contributions to the gluonic operators, eq. (4.41) and (4.42), are the same as in eq. (4.24), so that

$$
\left.\hat{\mathcal{C}}_{1}^{(7,0)}\right|_{n_{f}=5}=\left.\hat{\mathcal{C}}_{1}^{(7)}\right|_{n_{f}=5},\left.\quad \hat{\mathcal{C}}_{2}^{(8,1)}\right|_{n_{f}=5}=\left.\hat{\mathcal{C}}_{2}^{(7,0)}\right|_{n_{f}=5}
$$

The Wilson coefficients $\left.\hat{\mathcal{C}}_{3}^{(7,0)}\right|_{n_{f}=5}$ and $\left.\hat{\mathcal{C}}_{4}^{(8,1)}\right|_{n_{f}=5}$ vanish.

The Wilson coefficients for the scalar operators are

$$
\left.\hat{\mathcal{C}}_{5, f}^{(7,0)}\right|_{n_{f}=5}=\left.\hat{\mathcal{C}}_{5, f}^{(7)}\right|_{n_{f}=5},\left.\quad \hat{\mathcal{C}}_{6, f}^{(8,1)}\right|_{n_{f}=5}=\left.\frac{1}{m_{\chi}} \hat{\mathcal{C}}_{6, f}^{(7)}\right|_{n_{f}=5},
$$

while $\left.\hat{\mathcal{C}}_{7, f}^{(7,0)}\right|_{n_{f}=5}=\left.\hat{\mathcal{C}}_{8, f}^{(8,1)}\right|_{n_{f}=5}=0$. The dimension-five UV operators $Q_{3,4}^{(5)}$ in eq. (2.6) and $Q_{7,8}^{(5)}$ in eq. (2.8) contribute through a Higgs exchange at tree level, see figure 11 (right panel), and give the same matching conditions as in the case of light DM, eqs. (4.25) and (4.26). Note that within this subsection, the full (unexpanded) results of ref. [68] should be used.

The following twist-two operators are needed for the two-loop electroweak matching contributions (the numbering is chosen such that we avoid inconsistencies with the numbering in ref. [26]):

$$
\begin{aligned}
\mathcal{Q}_{23, q}^{(7,0)} & =\frac{1}{2}\left(\bar{\chi}_{v} \chi_{v}\right)\left[\bar{q}\left(\psi i \stackrel{\leftrightarrow}{D} \cdot v-\frac{1}{4} i \stackrel{\leftrightarrow}{D}\right) q\right] \\
\mathcal{Q}_{25}^{(7,0)} & =\left(\bar{\chi}_{v} \chi_{v}\right)\left[\frac{1}{4} G_{\alpha \beta}^{a} G^{a, \alpha \beta}-v_{\mu} v_{\nu} G^{a, \mu \lambda} G_{\lambda}^{a, \nu}\right] .
\end{aligned}
$$

The first operator, $\mathcal{Q}_{23, q}^{(7,0)}$, receives a non-vanishing matching contribution at the electroweak scale. It can be extracted from ref. [68] if in their results one takes the leading HDMET limit of the DM bilinears $\bar{\chi} i \partial^{\mu} \chi \rightarrow m_{\chi} \bar{\chi}_{v} v^{\mu} \chi_{v}$ and $\bar{\chi} \gamma^{\mu} \chi \rightarrow \bar{\chi}_{v} v^{\mu} \chi_{v}$. It is then given by $\mathcal{C}_{23, q}^{(7,0)}=g_{q}^{(1)}+g_{q}^{(2)}$, with the loop functions given in ref. [68]. The operator $\mathcal{Q}_{25}^{(7,0)}$ does not receive an initial condition at the weak scale, but is generated by QCD RG evolution below the weak scale, to be discussed in the following section. 

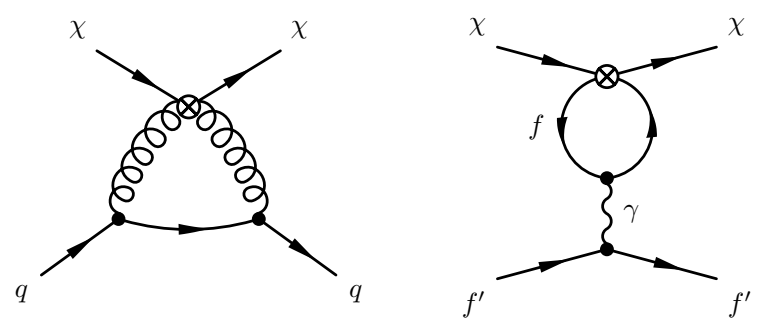

Figure 13. Left panel: the mixing of the gluonic operators into operators with scalar and pseudoscalar quark currents. Right panel: the mixing of dimension-six four-fermion operators into each other via the photon penguin insertion.

\subsection{RG running below the electroweak scale}

The matching at $\mu \sim \mu_{\mathrm{EW}}$ is followed by the QCD and QED RG running from $\mu_{\mathrm{EW}}$ to $\mu_{c} \sim \mu_{\text {had }} \sim 2 \mathrm{GeV}$. The five-flavor theory below $\mu_{\mathrm{EW}}$ is matched onto the four-flavor theory at the bottom quark threshold, $\mu_{b}$, and then onto the three-flavor theory at the charm quark threshold, $\mu_{c}$; see eq. (3.6). There is no running in the three flavor basis because of our choice of scales, $\mu_{c}=\mu_{\text {had }}$. This RG evolution was discussed in detail in ref. [16]. For completeness and convenience we convert the results of ref. [16] to our notation. (See also ref. [23] for a computer implementation of the RG evolution, as well as ref. [22] for the case of vector mediators.)

QCD running. Since the vector currents are conserved, $\hat{C}_{1, q}^{(6)}$ and $\hat{C}_{3, q}^{(6)}$ in eqs. (4.8) and (4.9) do not run. Moreover, the axial currents have vanishing anomalous dimensions at $\mathcal{O}\left(\alpha_{s}\right)$ and so the Wilson coefficients $\hat{C}_{2, q}^{(6)}$ and $\hat{C}_{4, q}^{(6)}$ in eqs. (4.8) and (4.9) do not run at one-loop order. At dimension seven, the only non-zero effect is the mixing of the gluonic operators, eqs. (4.10)-(4.11), into the scalar operators eq. (4.12) - see left panel in figure 13 - with anomalous dimension (cf. ref. [16])

$$
\left[\gamma_{s}^{(1)}\right]_{\mathcal{Q}_{1 \cdots 4}^{(7)} \times \mathcal{Q}_{5, q \cdots 8, q}^{(7)}}=8 \operatorname{diag}\left(C_{F}, C_{F},-1,-1\right),
$$

where $q$ runs over active quark flavors and $C_{F}=4 / 3$. This anomalous dimension arises at $\mathcal{O}\left(\alpha_{s}^{2}\right)$ since the $G G$ operators are defined with an additional factor of $\alpha_{s}$ to reflect the fact that they are loop generated, and thus,

$$
\left[\gamma_{s}^{(0)}\right]_{\mathcal{Q}_{1 \cdots 4}^{(7)} \times \mathcal{Q}_{5, q \cdots 8, q}^{(7)}}=0
$$

The mixing of the two operators $\mathcal{Q}_{23, q}^{(7,0)}$ and $\mathcal{Q}_{25}^{(7,0)}$ in eq. (4.47) is given by the anomalous dimension matrix

$$
\left[\gamma_{s}^{(0)}\right]_{\mathcal{Q}_{23, q}^{(7,0)} \mathcal{Q}_{25}^{(7,0)} \times \mathcal{Q}_{23, q}^{(7,0)} \mathcal{Q}_{25}^{(7,0)}}=\left(\begin{array}{cc}
\frac{64}{9} & -\frac{4}{3} \\
-\frac{64}{9} & \frac{4}{3} N_{f}
\end{array}\right)
$$

QED running. In general, the QED contribution to the RG evolution can be neglected due to the smallness of the electromagnetic coupling constant. The one exception are the off-diagonal mixings of the operators $\mathcal{Q}_{1, f}^{(6)}$ for different fermion flavors $f$ (and similarly 
for $\mathcal{Q}_{2, f}^{(6)}$ ), induced by the photon penguin diagrams, see figure 13 . These mixings lead to nonzero scattering on nuclei even if DM couples only to leptons at leading order [24, 89]. The conservation of parity forbids the mixing of $\mathcal{Q}_{1, f}^{(6)}$ into $\mathcal{Q}_{2, f}^{(6)}$ and vice versa. The required anomalous dimensions can be found in ref. [61], and are

$$
\left[\gamma_{e}^{(0)}\right]_{\mathcal{Q}_{1, f}^{(6)}, \mathcal{Q}_{1, f^{\prime}}^{(6)}}=\left[\gamma_{e}^{(0)}\right]_{\mathcal{Q}_{2, f}^{(6)}, \mathcal{Q}_{2 ; f^{\prime}}^{(6)}}=\frac{8}{3} Q_{f} Q_{f^{\prime}} N_{c}^{f},
$$

where $Q_{f}$ is the electric charges of the SM fermion $f$, while $N_{c}^{f}=1(3)$, if $f$ is a lepton (quark). In analogy with eq. (3.2), we use the notation

$$
\gamma_{e}=\frac{\alpha}{4 \pi} \gamma_{e}^{(0)}+\ldots
$$

where the ellipsis denotes higher orders.

Finite corrections arise at each heavy flavor threshold. Beside the usual threshold corrections to $\alpha_{s}$ (see, e.g., ref. [90]), there are also finite threshold corrections for the operators in eqs. (4.10)-(4.11), where at $\mu=\mu_{b}$,

$$
\begin{aligned}
& \left.\hat{\mathcal{C}}_{1(2)}^{(7)}\right|_{n_{f}=4}\left(\mu_{b}\right)=\left.\hat{\mathcal{C}}_{1(2)}^{(7)}\right|_{n_{f}=5}\left(\mu_{b}\right)-\left.\hat{\mathcal{C}}_{5, b(6, b)}^{(7)}\right|_{n_{f}=5}\left(\mu_{b}\right), \\
& \left.\hat{\mathcal{C}}_{3(4)}^{(7)}\right|_{n_{f}=4}\left(\mu_{b}\right)=\left.\hat{\mathcal{C}}_{3(4)}^{(7)}\right|_{n_{f}=5}\left(\mu_{b}\right)+\left.\hat{\mathcal{C}}_{7, b(8, b)}^{(7)}\right|_{n_{f}=5}\left(\mu_{b}\right),
\end{aligned}
$$

while at $\mu=\mu_{c}$,

$$
\begin{aligned}
& \left.\hat{\mathcal{C}}_{1(2)}^{(7)}\right|_{n_{f}=3}\left(\mu_{c}\right)=\left.\hat{\mathcal{C}}_{1(2)}^{(7)}\right|_{n_{f}=4}\left(\mu_{c}\right)-\hat{\mathcal{C}}_{5, c(6, c)}^{(7)} \mid n_{n_{f}=4}\left(\mu_{c}\right), \\
& \left.\hat{\mathcal{C}}_{3(4)}^{(7)}\right|_{n_{f}=3}\left(\mu_{c}\right)=\left.\hat{\mathcal{C}}_{3(4)}^{(7)}\right|_{n_{f}=4}\left(\mu_{c}\right)+\hat{\mathcal{C}}_{7, c(8, c)}^{(7)} \mid n_{f}=4\left(\mu_{c}\right),
\end{aligned}
$$

such that the effects of the heavy quarks appear, at low energies, as additional contributions to the gluonic operators, eqs. (4.10)-(4.11). All the other Wilson coefficients cross the thresholds continuously, $\left.\hat{\mathcal{C}}_{i}^{(d)}\right|_{n_{f}-1}=\left.\hat{\mathcal{C}}_{i}^{(d)}\right|_{n_{f}}$.

\subsection{DM interactions with nucleons and nuclei}

The final step in the RG evolution is the matching at $\mu \sim \mu_{\text {had }}$ onto an effective theory describing interactions of DM with nonrelativistic protons and neutrons. The momenta exchanged in direct detection experiments are $q \lesssim 200 \mathrm{MeV}$, with a typical value of $20-$ $60 \mathrm{MeV}$, which is well below the chiral symmetry breaking scale $4 \pi f_{\pi} \sim m_{N}$. One can thus use chiral perturbation theory (ChPT) to organize different contributions in terms of an expansion in $\left(q / 4 \pi f_{\pi}\right)^{n}$, see refs. [6, 11, 13, 18, 20, 21, 34, 91]. The leading-order contributions come from the interactions involving a DM field and a single nucleon inside the nucleus (these can still be coherently summed over all the neutrons and protons in the nucleus).

The effective Lagrangian for DM scattering on nonrelativistic nucleons (see refs. $[9,10$, $12,21])$

$$
\mathcal{L}_{\mathrm{NR}}=\sum_{i, N} c_{i}^{N}\left(q^{2}\right) \mathcal{O}_{i}^{N}
$$


contains 14 operators with up to two derivatives which are needed to describe the chirally leading interactions. The momentum-independent nonrelativistic operators are

$$
\mathcal{O}_{1}^{N}=\mathbb{1}_{\chi} \mathbb{1}_{N}, \quad \mathcal{O}_{4}^{N}=\vec{S}_{\chi} \cdot \vec{S}_{N},
$$

while the relevant subset of momentum-dependent operators consists of

$$
\begin{array}{rlrl}
\mathcal{O}_{5}^{N} & =\vec{S}_{\chi} \cdot\left(\vec{v}_{\perp} \times \frac{i \vec{q}}{m_{N}}\right) \mathbb{1}_{N}, & & \mathcal{O}_{6}^{N}=\left(\vec{S}_{\chi} \cdot \frac{\vec{q}}{m_{N}}\right)\left(\vec{S}_{N} \cdot \frac{\vec{q}}{m_{N}}\right), \\
\mathcal{O}_{7}^{N}=\mathbb{1}_{\chi}\left(\vec{S}_{N} \cdot \vec{v}_{\perp}\right), & \mathcal{O}_{8}^{N}=\left(\vec{S}_{\chi} \cdot \vec{v}_{\perp}\right) \mathbb{1}_{N}, \\
\mathcal{O}_{9}^{N}=\vec{S}_{\chi} \cdot\left(\frac{i \vec{q}}{m_{N}} \times \vec{S}_{N}\right), & \mathcal{O}_{11}^{N}=-\left(\vec{S}_{\chi} \cdot \frac{i \vec{q}}{m_{N}}\right) \mathbb{1}_{N},
\end{array}
$$

with $N=p, n$. We use the conventions of [21,23], so that

$$
\vec{q}=\vec{k}_{2}-\vec{k}_{1}=\vec{p}_{1}-\vec{p}_{2}, \quad \vec{v}_{\perp}=\frac{\vec{p}_{1}+\vec{p}_{2}}{2 m_{\chi}}-\frac{\vec{k}_{1}+\vec{k}_{2}}{2 m_{N}},
$$

where $\vec{p}_{1(2)}$ and $\vec{k}_{1(2)}$ are the incoming (outgoing) nucleon and DM three-momenta, respectively.

The coefficients of the two momentum-independent operators (4.57) are, schematically,

$$
\begin{aligned}
c_{1}^{N} & \sim \frac{C_{1, \ldots, 4, f ; 15,16}^{(6)}}{\Lambda^{2}}+\frac{2}{27} \frac{m_{N}}{\Lambda m_{h}^{2}} C_{3,4}^{(5)}+\frac{\sigma_{q}}{\Lambda m_{h}^{2}} C_{3,4}^{(5)}+\frac{\alpha}{\Lambda m_{\chi}} C_{1,2}^{(5)}, \\
c_{4}^{N} & \sim \frac{C_{5, \ldots, 8, f ; 17,18}^{(6)}}{\Lambda^{2}}+\frac{\alpha}{\Lambda m_{N}} C_{1,2}^{(5)} .
\end{aligned}
$$

At leading chiral order one also has the contributions from the operators with two derivatives, $\mathcal{O}_{5,6}^{N}$, whose coefficients are

$$
c_{5}^{N} \sim \delta_{N, p} \frac{\alpha m_{N}}{\Lambda q^{2}} C_{1,2}^{(5)}, \quad \quad c_{6}^{N} \sim \frac{m_{N}^{2}}{m_{\pi}^{2}} \frac{C_{5, \ldots, 8, f ; 17,18}^{(6)}}{\Lambda^{2}}+\frac{\alpha m_{N}}{\Lambda q^{2}} C_{1,2}^{(5)} .
$$

The sums in eqs. (4.62)-(4.64) are to be understood in the scaling sense, i.e., we only indicate a rough order of magnitude for the contribution of each of the UV Wilson coefficients, $C_{a}^{(d)}$. Above we equated the weak scale with $\mu_{\mathrm{EW}} \sim m_{h} \sim m_{Z}$. The complete expressions can be obtained, for instance, from refs. [21, 23], using the matching results given in sections 4.1 and 4.2. The additional contributions arising from the twist-two operators are collected in appendix B.

The $\mathcal{O}_{1}^{N}$ operator receives contributions from the vector $\times$ vector parts of the operators $Q_{1, f}^{(6)}, \ldots Q_{4, f}^{(6)}$, eqs. (2.9)-(2.12), and from tree-level $Z$ exchange due to the $Q_{15,16}^{(6)}$ operators, eqs. (2.16) and (2.17). The analogous operators with an axial-vector DM current, $Q_{5, f}^{(6)}, \ldots Q_{8, f}^{(6)}$ and $Q_{17,18}^{(6)}$, lead to spin-spin coupling in the nonrelativistic limit, and contribute to both $\mathcal{O}_{4}^{N}$ and $\mathcal{O}_{6}^{N}$. The two contributions are parametrically of the same order, since the coefficient $c_{6}^{N}$ is enhanced by the pion pole, which compensates the $\mathcal{O}\left(q^{2}\right)$ 
suppression of $\mathcal{O}_{6}^{N}$ for $q^{2} / m_{\pi}^{2} \sim \mathcal{O}(1)$ (numerically, the compensation is still only partial for electroweak scale DM [21]). The dipole operators $Q_{1,2}^{(5)}$ give contributions to all four nonrelativistic operators, while the scalar operators $Q_{3,4}^{(5)}$ give leading contributions only to $Q_{1}^{N}$, through tree-level Higgs exchange. The parameters $\sigma_{q}$ in eq. (4.62) are related to the matrix elements of $\bar{q} q$ quark scalar currents and are of order $\mathcal{O}(20-40) \mathrm{MeV}$.

The coefficients of the single-derivative operators, eqs. (4.59) and (4.60), are schematically

$$
\begin{aligned}
c_{7}^{N} \sim \frac{C_{1, \ldots, 4, f}^{(6)}}{\Lambda^{2}}, \\
c_{8}^{N} \sim \frac{C_{5, \ldots, 8, f}^{(6)}}{\Lambda^{2}}, \\
c_{9}^{N} \sim \frac{C_{5, \ldots, 8, f}^{(6)}}{\Lambda^{2}}+\frac{m_{N}}{m_{\chi}} \frac{C_{1, \ldots, 4, f}^{(6)}}{\Lambda^{2}}, \\
c_{11}^{N} \sim \frac{2}{27} \frac{m_{N}^{2}}{\Lambda m_{h}^{2} m_{\chi}} C_{7,8}^{(5)}+\frac{\sigma_{q} m_{N}}{\Lambda m_{h}^{2} m_{\chi}} C_{7,8}^{(5)}+\frac{\alpha m_{N}}{\Lambda q^{2}} \delta_{N, p} C_{5,6}^{(5)} .
\end{aligned}
$$

The coefficients $c_{7}^{N}$ and $c_{8}^{N}$ arise from vector $\times$ axial and axial $\times$ vector parts of the operators $Q_{1, f}^{(6)}, \ldots Q_{4, f}^{(6)}$ and $Q_{5, f}^{(6)}, \ldots Q_{8, f}^{(6)}$, respectively, while all of these contribute to $c_{9}^{N}$. Since these operators are momentum (velocity) suppressed, they will give subleading contributions to the scattering rates, unless the leading contributions (to $c_{1}^{N}$ from the vector $\times$ vector parts, and to $c_{4,6}^{N}$ from axial $\times$ axial parts) cancel. In the next section we will discuss in more detail how realistic this is. The operators $\mathcal{O}_{7}^{N}, \ldots, \mathcal{O}_{9}^{N}$, also receive contributions from $Q_{15}^{(6)}, \ldots Q_{18}^{(6)}$ due to $Z$ exchange, where no such cancellation can occur. These contributions are thus always subleading and were neglected in eqs. (4.65)-(4.67). The operators $Q_{7,8}^{(5)}$ lead to $q^{2}$-suppressed contributions to the scattering rate in the nonrelativistic limit, while $Q_{5,6}^{(5)}$ induce an electric dipole moment for DM, giving a $1 / q^{2}$-enhanced direct detection scattering rate [92].

If the EFT above the weak scale is extended to mass dimension seven, then also the nonrelativistic operators, $\mathcal{O}_{10}^{N}=-\mathbb{1}_{\chi}\left(\vec{S}_{N} \cdot i \vec{q} / m_{N}\right), \mathcal{O}_{12}^{N}=\vec{S}_{\chi} \cdot\left(\vec{S}_{N} \times \vec{v}_{\perp}\right), \mathcal{O}_{14}^{N}=$ $-\left(\vec{S}_{\chi} \cdot i \vec{q} / m_{N}\right)\left(\vec{S}_{N} \cdot \vec{v}_{\perp}\right)$ become phenomenologically important [26]. They arise from dimension-seven operators with tensor DM currents and from interactions of DM with the $G \tilde{G}$ current. The scaling estimates for the corresponding coefficients are $c_{10}^{N} \sim \mathcal{O}\left(m_{N} / \Lambda^{3}\right)$, $c_{12}^{N} \sim \mathcal{O}\left(m_{q} / \Lambda^{3}\right), c_{14}^{N} \sim \mathcal{O}\left(m_{N} / \Lambda^{3}\right)$, setting the dimensionless Wilson coefficients to unity.

Having obtained the coefficients in the effective Lagrangian for DM scattering on nonrelativistic nucleons, eq. (4.56), the final step is to calculate the DM-nucleus scattering cross section [9, 10, 12],

$$
\begin{aligned}
\frac{d \sigma}{d E_{R}}=\frac{2 m_{A}}{\left(2 j_{A}+1\right) v^{2}} \sum_{\tau, \tau^{\prime}}\left[R_{M}^{\tau \tau^{\prime}} W_{M}^{\tau \tau^{\prime}}\right. & +R_{\Sigma^{\prime \prime}}^{\tau \tau^{\prime}} W_{\Sigma^{\prime \prime}}^{\tau \tau^{\prime}}+R_{\Sigma^{\prime}}^{\tau \tau^{\prime}} W_{\Sigma^{\prime}}^{\tau \tau^{\prime}} \\
& \left.+\frac{\vec{q}^{2}}{m_{N}^{2}}\left(R_{\Delta}^{\tau \tau^{\prime}} W_{\Delta}^{\tau \tau^{\prime}}+R_{\Delta \Sigma^{\prime}}^{\tau \tau^{\prime}} W_{\Delta \Sigma^{\prime \prime}}^{\tau \tau^{\prime}}\right)\right]
\end{aligned}
$$


Here, $E_{R}$ is the recoil energy of the nucleus, $m_{A}$ the mass of the nucleus, $j_{A}$ its spin, and $v$ the initial DM velocity in the lab frame. The kinematic factors contain the $c_{i}^{N}$ coefficients,

$$
\begin{aligned}
R_{M}^{\tau \tau^{\prime}} & =c_{1}^{\tau} c_{1}^{\tau^{\prime}}+\frac{1}{4}\left(\frac{\vec{q}^{2}}{m_{N}^{2}} \vec{v}_{T}^{\perp 2} c_{5}^{\tau} c_{5}^{\tau^{\prime}}+\vec{v}_{T}^{\perp 2} c_{8}^{\tau} c_{8}^{\tau^{\prime}}+\frac{\vec{q}^{2}}{m_{N}^{2}} c_{11}^{\tau} c_{11}^{\tau^{\prime}}\right) \\
R_{\Sigma^{\prime \prime}}^{\tau \tau^{\prime}} & =\frac{1}{16}\left(c_{4}^{\tau} c_{4}^{\tau^{\prime}}+\frac{\vec{q}^{2}}{m_{N}^{2}}\left(c_{4}^{\tau} c_{6}^{\tau^{\prime}}+c_{6}^{\tau} c_{4}^{\tau^{\prime}}\right)+\frac{\vec{q}^{4}}{m_{N}^{4}} c_{6}^{\tau} c_{6}^{\tau^{\prime}}\right) \\
R_{\Sigma^{\prime}}^{\tau \tau^{\prime}} & =\frac{1}{8} \vec{v}_{T}^{2} c_{7}^{\tau} c_{7}^{\tau^{\prime}}+\frac{1}{16}\left(c_{4}^{\tau} c_{4}^{\tau^{\prime}}+\frac{\vec{q}^{2}}{m_{N}^{2}} c_{9}^{\tau} c_{9}^{\tau^{\prime}}\right) \\
R_{\Delta}^{\tau \tau^{\prime}} & =\frac{1}{4}\left(\frac{\vec{q}^{2}}{m_{N}^{2}} c_{5}^{\tau} c_{5}^{\tau^{\prime}}+c_{8}^{\tau} c_{8}^{\tau^{\prime}}\right) \\
R_{\Delta \Sigma^{\prime}}^{\tau \tau^{\prime}} & =\frac{1}{4}\left(c_{5}^{\tau} c_{4}^{\tau^{\prime}}-c_{8}^{\tau} c_{9}^{\tau^{\prime}}\right)
\end{aligned}
$$

where $\vec{v}_{T}^{\perp}$ is defined as in eq. (4.61), but with the nucleus replacing the nucleon. The sum in (4.69) is over isospin, $\tau, \tau^{\prime}=0,1$, so that

$$
c_{i}^{0(1)}=\frac{1}{2}\left(c_{i}^{p} \pm c_{i}^{n}\right) .
$$

The nuclear response functions depend on $|\vec{q}|$ and have the approximate scaling (see, e.g., figure 2 in [93])

$$
W_{M} \sim \mathcal{O}\left(A^{2}\right), \quad W_{\Sigma^{\prime}}, W_{\Sigma^{\prime \prime}}, W_{\Delta}, W_{\Delta \Sigma^{\prime}} \sim \mathcal{O}\left(10^{-2}\right)-\mathcal{O}(1) .
$$

The $W_{\Sigma^{\prime}}, W_{\Sigma^{\prime \prime}}, W_{\Delta}$, and $W_{\Delta \Sigma^{\prime}}$ response functions depend strongly on the detailed properties of nuclei, for instance, whether or not they have an un-paired nucleon in the outer shell. Here $W_{\Sigma^{\prime}, \Sigma^{\prime \prime}}$ measure the spin content of the nucleus, $W_{\Delta}$ the average angular momentum in the nucleus, and $W_{\Delta \Sigma^{\prime}}$ the interference of the two. Their sizes can thus differ drastically between different isotopes of the same element.

The $W_{M}$ response function encodes the coherent scattering enhancement, $\mathcal{O}\left(A^{2}\right)$, where $A$ is the atomic mass number. This is achieved in the long-wavelength limit, $q \rightarrow 0$, where DM scatters coherently on the whole nucleus, for instance, due to the $\mathcal{O}_{1}^{N}$ contact interaction. The coherent scattering due to $\mathcal{O}_{5}^{N}$ is $\mathcal{O}\left(q^{2} v_{T}^{2}\right)$ suppressed. However, since its coefficient is $1 / q^{2}$ enhanced, the corresponding contribution is of leading order [21]. The contributions due to $\mathcal{O}_{8,11}^{N}$, though coherently enhanced, are at the same time velocity suppressed.

\section{The effects of RG running}

The impact of the mixing of electroweak operators on the scattering cross section depends on two factors: i) the structure of the anomalous dimension and thus the sizes of the induced Wilson coefficients in eqs. (2.5)-(2.17), and ii) on the sizes of the nuclear response functions, eq. (4.76), for each of the operators involved in the mixing. In section 5.1 we first give the scalings of the scattering cross sections without mixing effects, for several benchmark choices of UV Wilson coefficients. In section 5.2 we then include the mixing and perform the actual numerical analysis using the full expressions for the DM-nucleus scattering cross sections derived in the sections above. 


\subsection{Low energy phenomenology ignoring RG running}

We first estimate the size of the DM-nucleus scattering cross section induced by each of the UV operators, eqs. (2.5)-(2.8) and (2.9)-(2.17), neglecting the RG running. In the estimates we use the scaling for nonrelativistic Wilson coefficients in eqs. (4.62)-(4.68), and the rough scalings of nuclear response functions in eq. (4.76), but setting for simplicity $W_{\Sigma^{\prime}}, W_{\Sigma^{\prime \prime}}, W_{\Delta}, W_{\Delta \Sigma^{\prime}} \sim \mathcal{O}(1)$, with the knowledge that the sizes of the latter contributions have high variations between different target materials.

\subsubsection{Magnetic or electric dipole operators}

The DM magnetic dipole operators, $Q_{1,2}^{(5)} \sim\left(\bar{\chi} \sigma_{\mu \nu} \chi\right)\left\{B^{\mu \nu}, W^{\mu \nu}\right\}$, eq. (2.5), induce both spin-dependent and spin-independent interactions. These give parametrically similar contributions to the DM-nucleus scattering cross section. Schematically,

$$
\begin{aligned}
\frac{d \sigma}{d E_{R}} & \sim\left(c_{1}^{2}+\vec{v}_{T}^{\perp 2} \frac{\vec{q}^{2}}{m_{N}^{2}} c_{5}^{2}\right) W_{M}+\left\{c_{4}, \frac{\vec{q}^{2}}{m_{N}^{2}} c_{6}\right\}^{2} W_{\Sigma^{\prime}, \Sigma^{\prime \prime}}+\left\{\frac{\vec{q}^{2}}{m_{N}^{2}} c_{5}, c_{4}\right\}^{2} W_{\Delta, \Delta \Sigma^{\prime}} \\
& \sim\left(\frac{\alpha C_{1,2}^{(5)}}{\Lambda}\right)^{2}\left[\left(\frac{1}{m_{\chi}^{2}}+\frac{\vec{v}_{T}^{\perp^{2}}}{\vec{q}^{2}}\right) A^{2}+\frac{1}{m_{N}^{2}}+\frac{1}{m_{N}^{2}}\right],
\end{aligned}
$$

where we shortened the notation, $c_{i}^{N} \rightarrow c_{i}$, and dropped common factors. The scaling estimates for each of the three terms are given in the second line. The spin-independent scattering has two contributions, both $\mathcal{O}\left(A^{2}\right)$ coherently enhanced: the contribution from $\mathcal{O}_{1}^{N}$ is suppressed by $\mathcal{O}\left(1 / m_{\chi}^{2}\right)$, while the contribution from $\mathcal{O}_{5}^{p}$ contains a photon pole, leading to a net suppression of $\mathcal{O}\left(\vec{v}_{T}^{\perp} / \vec{q}^{2}\right)$. Using $\left|\vec{v}_{T}^{\perp}\right| \sim 10^{-3},|\vec{q}| \sim 0.1 m_{N}$, the two contributions are comparable for $m_{\chi} \sim \mathcal{O}(100 \mathrm{GeV})$. The two spin-dependent terms carry a much smaller mass suppression of $\mathcal{O}\left(1 / m_{N}^{2}\right)$, but no coherent enhancement. Which term dominates then depends on the details of the nuclear structure for the nuclei in the target [21].

The DM electric dipole operators, $Q_{5,6}^{(5)} \sim\left(\bar{\chi} \sigma_{\mu \nu} \gamma_{5} \chi\right)\left\{B^{\mu \nu}, W^{\mu \nu}\right\}$, eq. (2.7), match onto the nuclear operator $\mathcal{O}_{11}^{p} \sim i \vec{q} \cdot \vec{S}_{\chi}$. This leads to coherently enhanced scattering independent of the nuclear spin, with the $1 /|\vec{q}|^{2}$ pole only partially cancelled,

$$
\frac{d \sigma}{d E_{R}} \sim \frac{\vec{q}^{2}}{m_{N}^{2}}\left(c_{11}^{p}\right)^{2} W_{M} \sim\left(\frac{\alpha}{\Lambda} \frac{1}{|\vec{q}|} C_{5,6}^{(5)}\right)^{2} A^{2} .
$$

Compared to the magnetic dipole operators, the bounds on the NP scale $\Lambda$ for electric dipole interactions of DM are thus more stringent by a factor of order $m_{\chi} /|\vec{q}|$.

\subsubsection{Operators with DM scalar currents}

The operators $Q_{3,4}^{(5)} \sim(\bar{\chi} \chi)\left(H^{\dagger} H\right)$, eq. (2.6), generate DM interactions with a scalar quark current once the Higgs is integrated out at $\mu \sim m_{Z}$. Integrating out the top, bottom, and charm quarks at the respective thresholds generates an effective coupling of DM to gluons. At $\mu \sim \mu_{\text {had }}$ DM thus couples to both the gluonic and light-quark scalar currents. 
Both of these match onto the nuclear operator $\mathcal{O}_{1}^{N} \sim \mathbb{1}_{\chi} \mathbb{1}_{N}$, giving a coherently enhanced spin-independent cross section,

$$
\frac{d \sigma}{d E_{R}} \sim c_{1}^{2} W_{M} \sim\left(\frac{2}{27} \frac{m_{N}}{\Lambda m_{h}^{2}} C_{3,4}^{(5)}\right)^{2} A^{2},
$$

where in the last term we kept the numerically important factor $2 / 27$.

The operators with pseudoscalar DM current, $Q_{7,8}^{(5)} \sim\left(\bar{\chi} \gamma_{5} \chi\right)\left(H^{\dagger} H\right)$, eq. (2.8), follow a similar series of matchings. The only significant difference arises in the nonrelativistic limit, where the DM pseudoscalar current gives an $\mathcal{O}(q)$ suppressed operator, $\mathcal{O}_{11}^{N} \sim \vec{i} q \cdot \vec{S}_{\chi}$. The resulting DM-nucleus scattering cross section is still coherently enhanced, but suppressed by $\mathcal{O}\left(\vec{q}^{2} / m_{\chi}^{2}\right)$ compared to $(5.3)$,

$$
\frac{d \sigma}{d E_{R}} \sim \frac{\vec{q}^{2}}{m_{N}^{2}} c_{11}^{2} W_{M} \sim \frac{\vec{q}^{2}}{m_{\chi}^{2}}\left(\frac{2}{27} \frac{m_{N}}{\Lambda m_{h}^{2}} C_{7,8}^{(5)}\right)^{2} A^{2} .
$$

\subsubsection{Operators with DM vector current and with quark vector or axial-vector currents}

We focus next on the operators $Q_{1, i}^{(6)}, \ldots, Q_{4, i}^{(6)} \sim\left(\bar{\chi} \gamma^{\mu} \chi\right)\left\{\bar{q}_{L} \gamma_{\mu} q_{L}, q_{R} \gamma_{\mu} q_{R}\right\}$, eqs. (2.9)-(2.12). Barring cancellations, the leading contribution is due to the vector $\times$ vector part of the operators, $\left(\bar{\chi} \gamma_{\mu} \chi\right)\left(\bar{q} \gamma^{\mu} q\right)$. For couplings to the first generation quarks this leads to coherently enhanced spin-independent scattering,

$$
\frac{d \sigma}{d E_{R}} \sim c_{1}^{2} W_{M} \sim\left(\frac{1}{\Lambda^{2}} C_{1 q, \ldots, 4 q}^{(6)}\right)^{2} A^{2} \quad\left(1^{\text {st }} \text { generation quarks }\right) .
$$

The estimate is different, if DM only couples to quarks of the second or third generation. For these the nuclear matrix element of the vector current vanishes, and the leading contribution comes from closing the quarks in a loop, exchanging a photon with the upor down-quark vector currents. This also results in a spin-independent scattering, with a cross section

$$
\frac{d \sigma}{d E_{R}} \sim\left(\frac{\alpha}{4 \pi} \frac{1}{\Lambda^{2}} C_{1 q, \ldots, 4 q}^{(6)}\right)^{2} A^{2} \quad\left(2^{\text {nd }} \text { and } 3^{\text {rd }} \text { generation quarks }\right) .
$$

In addition there are subleading contributions from matching onto higher dimension operators with gluons, as well as spin-dependent, velocity-suppressed scattering from the axial currents.

The situation is qualitatively different if the UV physics is such that at $\mu \sim \Lambda$ it projects the $Q_{1, i}^{(6)}, \ldots, Q_{4, i}^{(6)}$ operators only on the vector $\times$ axial-vector structure. For instance, if the Wilson coefficients obey $C_{1, i}^{(6)}(\Lambda)=0$, while $C_{2, i}^{(6)}(\Lambda)=-C_{3, i}^{(6)}(\Lambda)=-C_{4, i}^{(6)}(\Lambda)$, then, neglecting RG effects, only operators of the form $\left(\bar{\chi} \gamma_{\mu} \chi\right)\left(\bar{q} \gamma^{\mu} \gamma_{5} q\right)$ are generated. If the operators involve light quarks, this gives a spin-dependent cross section that scales as (for $q=u, d, s)$

$$
\frac{d \sigma}{d E_{R}} \sim\left(\vec{v}_{T}^{\perp 2} c_{7}^{2}+\frac{\vec{q}^{2}}{m_{N}^{2}} c_{9}^{2}\right) W_{\Sigma^{\prime}} \sim\left(\vec{v}_{T}^{2}+\frac{\vec{q}^{2}}{m_{\chi}^{2}}\right)\left(\frac{\mathcal{C}_{1 i, \ldots, 4 i}^{(6)}}{\Lambda^{2}}\right)^{2}, \quad \text { (axial vector) }
$$


The two contributions are comparable for $|\vec{q}| \sim 0.1 m_{N}$ and $m_{\chi} \sim \mathcal{O}(100 \mathrm{GeV})$. If the vector $\times$ axial-vector operators involve only the heavy quarks, $q=c, b, t$, the scattering cross section is further severely suppressed by the small contributions of the heavy quarks to the nucleon spin (see section 5.1.4 below and ref. [25] for a more detailed discussion).

Note that the spin-dependent scattering in eq. (5.7) is suppressed by $\vec{v}_{T}^{2} \sim \vec{q}^{2} / m_{\chi}^{2} \sim$ $10^{-6}$. There is no such suppression for the spin-independent cross section, eq. (5.5), which is, in addition, enhanced by the coherence factor $A^{2}$. This means that the Wilson coefficients contributing to the quark vector currents at the scale $\mu \gtrsim m_{Z}$ need to cancel to the level $\sim|\vec{q}| /\left(m_{\chi} A\right) \sim \mathcal{O}\left(10^{-6}\right)$ if the spin-dependent scattering is to be the dominant DM-nucleus interaction. Perfect cancellation at all scales is impossible to arrange, since the contributions come from operators in different representations of the SM gauge group, $\left(\bar{\chi} \gamma_{\mu} \chi\right)\left(\bar{Q}_{L} \gamma^{\mu} Q_{L}\right),\left(\bar{\chi} \gamma_{\mu} \chi\right)\left(\bar{u}_{R} \gamma^{\mu} u_{R}\right),\left(\bar{\chi} \gamma_{\mu} \chi\right)\left(\bar{d}_{R} \gamma^{\mu} d_{R}\right)$. Even if one engineers the Wilson coefficients of these operators such that the vector currents are zero at one scale, a small amount of running will make them nonzero at a different scale. The required cancellation is numerically of three-loop order, so that even the radiative corrections may need to be canceled by fine tuning in order for the spin-dependent scattering to be the leading effect.

\subsubsection{Operators with DM axial-vector and with quark vector or axial-vector currents}

A qualitatively different situation is encountered for the operators that involve DM axial-vector currents, $Q_{5, i}^{(6)}, \ldots, Q_{8, i}^{(6)} \sim\left(\bar{\chi} \gamma_{\mu} \gamma_{5} \chi\right)\left\{\bar{q}_{L} \gamma^{\mu} q_{L}, \bar{q}_{R} \gamma^{\mu} q_{R}\right\}$, eqs. (2.9)-(2.12). In this case the $\left(\bar{\chi} \gamma_{\mu} \gamma_{5} \chi\right)\left(\bar{q} \gamma^{\mu} \gamma_{5} q\right)$ operators lead to spin-dependent scattering, while the $\left(\bar{\chi} \gamma_{\mu} \gamma_{5} \chi\right)\left(\bar{q} \gamma^{\mu} q\right)$ operators lead to coherently enhanced, but momentum-suppressed scattering. We discuss each of the two limiting cases separately.

If the operator $\left(\bar{\chi} \gamma_{\mu} \gamma_{5} \chi\right)\left(\bar{q} \gamma^{\mu} \gamma_{5} q\right)$ involves light quarks, $q=u, d, s$, this results in a spin-dependent cross section (not displaying explicitly the suppression for strange quark due to its small axial charge, $\Delta s=-0.031(5)[21,94-97])$,

$$
\frac{d \sigma}{d E_{R}} \sim\left\{c_{4}, \frac{\vec{q}^{2}}{m_{N}^{2}} c_{6}\right\}^{2} W_{\Sigma^{\prime}, \Sigma^{\prime \prime}} \sim\left(\frac{C_{5 i, \ldots, 8 i}^{(6)}}{\Lambda^{2}}\right)^{2} \quad \text { (light quarks) }
$$

If the operator $\left(\bar{\chi} \gamma_{\mu} \gamma_{5} \chi\right)\left(\bar{q} \gamma^{\mu} \gamma_{5} q\right)$ involves only the heavy quarks, $q=t, b, c$, the scattering cross section is generally very small. The axial charges of charm and bottom quarks are tiny and poorly determined. Ref. [98] obtained $\Delta c \approx-5 \cdot 10^{-4}, \Delta b \approx-5 \cdot 10^{-5}$, with probably at least a factor of two uncertainty on these estimates. Despite this, for heavy quark axial-axial interactions the heavy quark axial charges still dominate the cross section over the contributions from mixing induced couplings to light quarks, discussed in the next section (see also ref. [25]).

We focus next on the limiting case where at $\mu \sim m_{Z}$ only the axial-vector $\times$ vector operators, $\left(\bar{\chi} \gamma_{\mu} \gamma_{5} \chi\right)\left(\bar{q} \gamma^{\mu} q\right)$, are generated. For $q=u, d, s$ these match on two nonrelativistic operators with one derivative, $\mathcal{O}_{8}^{N}, \mathcal{O}_{9}^{N}$. Both lead to momentum suppressed incoherent scattering, with $\mathcal{O}_{8}^{N}$ giving rise, in addition, to spin-independent scattering that is coher- 
ently enhanced, but velocity suppressed,

$$
\begin{aligned}
\frac{d \sigma}{d E_{R}} & \sim \vec{v}_{T}^{\perp 2} c_{8}^{2} W_{M}+\frac{\vec{q}^{2}}{m_{N}^{2}}\left\{c_{8}, c_{9}\right\}^{2} W_{\Delta, \Delta \Sigma^{\prime}, \Sigma^{\prime}} \\
& \sim\left\{\vec{v}_{T}^{\perp 2} A^{2}, \frac{\vec{q}^{2}}{m_{N}^{2}}\right\}\left(\frac{C_{5 i, \ldots, 8 i}^{(6)}}{\Lambda^{2}}\right)^{2} \quad \text { (light quarks) }
\end{aligned}
$$

The two contributions are of parametrically similar size for heavy nuclei, $A \sim \mathcal{O}(100)$, in which case $\left|\vec{v}_{T}\right| A \sim|\vec{q}| / m_{N}$. Which of the two contributions dominates then depends on the details of the nuclear structure for the particular isotope.

For $\left(\bar{\chi} \gamma_{\mu} \gamma_{5} \chi\right)\left(\bar{q} \gamma^{\mu} q\right)$ with $q=t, b, c$, the leading contribution comes from closing the heavy quark loop, exchanging a photon with the up- or down-quark vector current. The cross section is suppressed with respect to eq. (5.9) by an additional factor of $(\alpha / 4 \pi)^{2}$,

$$
\frac{d \sigma}{d E_{R}} \sim\left(\frac{\alpha}{4 \pi}\right)^{2}\left\{\vec{v}_{T}^{\perp 2} A^{2}, \frac{\vec{q}^{2}}{m_{N}^{2}}\right\}\left(\frac{C_{5 i, \ldots, 8 i}^{(6)}}{\Lambda^{2}}\right)^{2} \quad \text { (heavy quarks) }
$$

There is also a contribution from matching onto higher dimension operators with gluons, which is expected to be at most of similar size.

In general the sum of $Q_{5, i}^{(6)}, \ldots, Q_{8, i}^{(6)}$ operators matches onto both $\left(\bar{\chi} \gamma_{\mu} \gamma_{5} \chi\right)\left(\bar{q} \gamma^{\mu} \gamma_{5} q\right)$ and $\left(\bar{\chi} \gamma_{\mu} \gamma_{5} \chi\right)\left(\bar{q} \gamma^{\mu} q\right)$ operators at $\mu=\mu_{\mathrm{EW}}$, giving a cross section that is a sum of eqs. (5.8) and (5.9). The spin-dependent scattering in eq. (5.8) is parametrically the largest. Since the parametric enhancement is not large, however, this expectation does depend on the target material, and spin-independent scattering could be equally important.

\subsubsection{Operators with Higgs vector currents}

The operators $Q_{15,16}^{(6)} \sim\left(\bar{\chi} \gamma^{\mu} \chi\right)\left(H^{\dagger} D_{\mu} H\right)$ and $Q_{17,18}^{(6)} \sim\left(\bar{\chi} \gamma^{\mu} \gamma_{5} \chi\right)\left(H^{\dagger} D_{\mu} H\right)$, eqs. (2.16) and (2.17), give rise to a DM-DM- $Z$ boson vertices after the Higgs obtains its vacuum expectation value. Integrating out the $Z$ at $\mu \sim m_{Z}$ leads to a coupling of DM to vector and axial-vector quark currents. The relative strength of the two is fixed by the $Z$ couplings to the left- and right-handed quarks. This is different from the case of the operators $Q_{1, i}^{(6)}, \ldots, Q_{8, i}^{(6)}$ that we discussed before, where a more general structure of DM couplings to quarks was allowed.

For the operators $Q_{15,16}^{(6)} \sim\left(\bar{\chi} \gamma^{\mu} \chi\right)\left(H^{\dagger} D_{\mu} H\right)$, the dominant contribution comes from a quark vector current, giving a coherently enhanced, spin-independent scattering cross section

$$
\frac{d \sigma}{d E_{R}} \propto c_{1}^{2} W_{M} \sim\left(\frac{1}{\Lambda^{2}} C_{15,16}^{(6)}\right)^{2} A^{2}
$$

The $Z$-boson exchange at $\mu \sim m_{Z}$ also generates the $\left(\bar{\chi} \gamma_{\mu} \chi\right)\left(\bar{q} \gamma^{\mu} \gamma_{5} q\right)$ operator. This leads to momentum-suppressed, spin-dependent scattering that is always subleading.

On the other hand, for the operators with DM axial-vector currents, $Q_{17,18}^{(6)} \sim$ $\left(\bar{\chi} \gamma^{\mu} \gamma_{5} \chi\right)\left(H^{\dagger} D_{\mu} H\right)$, one needs to keep both the spin-dependent and spin-independent scattering contributions. The induced axial-vector $\times$ vector and axial-vector $\times$ axial-vector inter- 
actions lead to a cross section that scales as the sum of eqs. (5.8) and (5.9),

$$
\begin{aligned}
\frac{d \sigma}{d E_{R}} & \sim\left\{c_{4}, \frac{\vec{q}^{2}}{m_{N}^{2}} c_{6}\right\}^{2} W_{\Sigma^{\prime}, \Sigma^{\prime \prime}}+\vec{v}_{T}^{\perp 2} c_{8}^{2} W_{M}+\frac{\vec{q}^{2}}{m_{N}^{2}}\left\{c_{8}, c_{9}\right\}^{2} W_{\Delta, \Delta \Sigma^{\prime}, \Sigma^{\prime}} \\
& \sim\left(1+\vec{v}_{T}^{\perp 2} A^{2}+\frac{\vec{q}^{2}}{m_{N}^{2}}\right)\left(\frac{C_{17,18}^{(6)}}{\Lambda^{2}}\right)^{2} .
\end{aligned}
$$

From scaling considerations, spin-dependent scattering is expected to be dominant in nuclei with an unpaired nucleon that is not in an $s$-shell. But even then the spin-independent scattering contributions may need to be included, depending on the nucleus. An example is dicsussed in section 5.2.1.

\subsection{Inclusion of RG running}

The modifications due to RG running can significantly impact the cross section predictions. We will show several examples where the RG running effects are particularly large. While the sizes and patterns of the induced corrections does depend on the electroweak charges of DM, the effects themselves are not "optional". They are due to SM particles in the loops, and are thus always present.

Consider, for instance, $\mathrm{SU}(2)_{L}$-singlet $\mathrm{DM}$, where all mixing proportional to $g_{2}$ vanishes, as can be seen by inspecting $\gamma_{2}^{(0)}$. Another example is DM that is hypercharge neutral, $Y_{\chi}=0$, for which all the mixings due to $B_{\mu}$ exchanges with the DM line vanish. However, in both cases there is still mixing due to the running of the non-conserved SM currents. For instance, for DM that is a complete SM singlet the main mixing is induced by the top-quark Yukawa interaction. This case has been discussed in detail in the literature [22, 60, 61] (see also ref. [25] for the discussion of weak-mixing effects below the weak scale).

Here, we will use our general results from section 3 and apply them to the simplest nontrivial example of DM with electroweak charges - a Dirac fermion multiplet that is hypercharge neutral, $Y_{\chi}=0$, and an electroweak triplet, $I_{\chi}=1$. The choice $Y_{\chi}=0$ is imposed on us by the phenomelogical requirement that DM (the neutral component of the multiplet) should not couple to the $Z$ boson at tree level, in order to avoid a too large direct detection scattering scattering cross section.

We will illustrate the effects of RG running for several different choices of nonrenormalizable DM interactions, taking $I_{\chi}=1$ as an example. The scattering rates then receive two types of contributions. First, there are contributions from higher dimension operators. These vanish in the limit $\Lambda \rightarrow \infty$. However, for $I_{\chi} \neq 0$ there are also contributions from renormalizable electroweak interactions that are independent of $\Lambda$. The leading contributions of this type are due to the small "Higgs penguin", the one-loop and two-loop contributions shown in figure 2. They lead to coherently enhanced scattering of parametric size (for $I_{\chi}=1$, using the right diagram in figure 2)

$$
\left.\frac{d \sigma}{d E_{R}}\right|_{\text {"Higgs penguin" }} \propto A^{2}\left(\frac{\alpha_{2}}{4 \pi}\right)^{4} \frac{g_{s}^{4}}{M_{W}^{4}} \sim \frac{10^{-19} A^{2}}{\mathrm{GeV}^{4}},
$$


where $A^{2}=\mathcal{O}\left(10^{4}\right)$ for scattering on Xenon, and $A^{2}=\mathcal{O}\left(10^{2}\right)$ for scattering on Fluorine. If the target nucleus has non-zero spin, the $W$ box shown in figure 2 gives an additional contribution scaling as

$$
\left.\frac{d \sigma}{d E_{R}}\right|_{\text {"W box" }} \propto\left(\frac{\alpha_{2}}{4 \pi}\right)^{4} \frac{1}{M_{W}^{4}} \sim \frac{10^{-16}}{\mathrm{GeV}^{4}} .
$$

These scalings omit a proportionality factor that depends on the DM mass and velocity, the recoil energy, and the detailed structure of the nucleus. For a typical scattering event with $m_{\chi}=100 \mathrm{GeV}$ and $E_{R}=20 \mathrm{keV}$, this factor is roughly of the order of $10^{8} \mathrm{GeV}$. In our numerical evaluations we use the exact results from ref. [68] (for heavy DM see also $[7,27,81])$. The ratio of scaling estimates in eqs. (5.13) and (5.14) agrees with the ratio of full results within an order of magnitude. Note that for DM that is a complete electroweak singlet the gauge contribution is absent.

In figures 14 to 16 we show numerical examples for DM scattering rates in two fictitious, yet realistic detectors. For a Xenon target we integrate the differential rates over $E_{R} \in[5 \mathrm{keV}, 40 \mathrm{keV}]$, and for Fluorine over $E_{R} \in[3.3 \mathrm{keV}, 200 \mathrm{keV}]$. We average over the natural abundances of the xenon isotopes and assume a standard Maxwell-Boltzmann velocity distribution with mean velocity $240 \mathrm{~km} / \mathrm{s}$. For nuclear response functions we use the predictions of refs. $[9,12]$, while for nuclear form factors we use the inputs collected in ref. [21]. In the figures the DM mass varies in the range $m_{\chi} \in[30 \mathrm{GeV}, 1 \mathrm{TeV}]$. While in the lower part of the range the shown benchmarks are likely excluded by LEP constraints and LHC searches, we keep them for illustration purposes.

In the three examples that we show below the effective interactions involve axial-vector quark currents. The reason for this choice is easy to understand. In the case where we have DM current coupling to either only LH or only RH quarks, the vector-vector part of the interaction always dominates, and the RG effects are subdominant. In the case where we have DM axial-vector current coupling to either only LH or only RH quarks, the mixing effects are larger and are $\mathcal{O}(1)$. We instead show the cases where the RG running induces the largest corrections, i.e., the case of DM interacting with axial-vector quark currents.

In all our examples DM couples to both up- and down-type quarks. Using the triplet operators $Q_{1, i}^{(6)}$ and $Q_{5, i}^{(6)}$ it is possible to construct interactions of DM with only up- or down-quark currents separately. This would, however, require a nonzero DM hypercharge, $Y_{\chi} \neq 0$, which is phenomenologically not viable for Dirac fermion DM.

\subsubsection{Operators with DM axial-vector current and 3rd generation quark axial- vector current}

For the first example we assume that at the high scale, $\Lambda$, the only nonzero Wilson coefficients are

$$
C_{6,3}^{(6)}(\Lambda)=-C_{7,3}^{(6)}(\Lambda)=-C_{8,3}^{(6)}(\Lambda) .
$$

That is, we assume that DM couples to the SM through renormalizable weak interactions $\left(I_{\chi}=1, Y_{\chi}=0\right)$ and, in addition, through the dimension 6 effective operator

$$
-Q_{6,3}^{(6)}+Q_{7,3}^{(6)}+Q_{8,3}^{(6)}=\left(\bar{\chi} \gamma_{\mu} \gamma_{5} \chi\right)\left(\bar{t} \gamma^{\mu} \gamma_{5} t+\bar{b} \gamma^{\mu} \gamma_{5} b\right) .
$$


At tree-level this operator has vanishingly small nuclear matrix element, see section 5.1. Appreciable DM-nucleus scattering is generated only once we close the heavy quark loop. The RG running captures the logarithmically enhanced part of this contribution. Starting with

$$
-C_{6,3}^{(6)}(\Lambda)=C_{7,3}^{(6)}(\Lambda)=C_{7,3}^{(6)}(\Lambda)=1
$$

the RG running from $\Lambda$ to $\mu \sim M_{W}$ generates the Wilson coefficients (keeping only the linear logarithmic term)

$$
\begin{aligned}
C_{1,3}^{(6)}\left(m_{W}\right) & =-\frac{g_{2}^{2}}{16 \pi^{2}}\left[\gamma_{2}^{(0)}\right]_{Q_{6,3}^{(6)}, Q_{1,3}^{(6)}} \log \frac{m_{W}}{\Lambda}=\frac{g_{2}^{2}}{16 \pi^{2}} 12 \log \frac{m_{W}}{\Lambda} \\
C_{18}^{(6)}\left(m_{W}\right) & =\frac{y_{t}^{2}}{16 \pi^{2}}\left(-\left[\gamma_{y_{t}}^{(0)}\right]_{Q_{6,3}^{(6)}, Q_{18}^{(6)}}+\left[\gamma_{y_{t}}^{(0)}\right]_{Q_{7,3}^{(6)}, Q_{18}^{(6)}}\right) \log \frac{m_{W}}{\Lambda} \\
& =-\frac{y_{t}^{2}}{16 \pi^{2}} 4 N_{c} \log \frac{m_{W}}{\Lambda} .
\end{aligned}
$$

In deriving eqs. (5.18) and (5.19) we took into account the cancelations of contributions that arise due to the actual values of the anomalous dimensions. For instance, the mixing via penguin insertions generically results in DM coupling to the first two generations of quarks by generating the operators $Q_{6, i}^{(6)}, \ldots, Q_{8, i}^{(6)}$. However, for the initial conditions in eq. (5.16) this mixing vanishes at leading-logarithmic order. There are also mixings into higher dimension operators coupling DM to photons or gluons instead of quark currents. These involve at least two gauge field strengths and an additional derivative, so that the scattering contributions are further power suppressed. The leading contributions to the scattering rates therefore come from the mixing induced operators $Q_{1,3}^{(6)}$ and $Q_{18}^{(6)}$. The mixing into $Q_{18}^{(6)}$ is due to the top-quark Yukawa interaction of the Higgs. It is present whether or not DM is part of an electroweak multiplet, i.e., even if DM is an electroweak singlet.

In figure 14 we compare the predicted rates for scattering on Xenon and Fluorine, obtained with (blue lines) and without (red lines) RG evolution. In the case of no RG evolution the scattering is almost entirely due to the contribution of renormalizable weak interactions. For $\Lambda=1 \mathrm{TeV}$ the $\mathrm{RG}$ induced contributions from dimension-six operators dominate over the renormalizable ones, in the case of Fluorine by up to two orders of magnitude.

The sizes of the different contributions can be qualitatively understood from their parametric scalings, given for the gauge contributions in eqs. (5.13) and (5.14). The mixing induced $Q_{18}^{(6)}$ leads to a cross section that scales roughly as

$$
\frac{d \sigma}{d E_{R}} \propto\left[1+\frac{q^{2}}{m_{N}^{2}}+\left(v_{T}^{\perp}\right)^{2} A^{2}\right]\left(\frac{\alpha_{t}}{4 \pi}\right)^{2}\left(12 \log \frac{M_{W}}{\Lambda}\right)^{2} \frac{1}{\Lambda^{4}} \sim \frac{10^{-14}+10^{-18}+10^{-20} A^{2}}{\mathrm{GeV}^{4}},
$$

while the cross section induced by the mixing into $Q_{1,3}^{(6)}$, scales roughly as

$$
\frac{d \sigma}{d E_{R}} \propto A^{2}\left(\frac{\alpha_{2}}{4 \pi}\right)^{2}\left(12 \log \frac{M_{W}}{\Lambda}\right)^{2}\left(\frac{\alpha}{4 \pi}\right)^{2}\left(\frac{16}{9} \log \frac{m_{b}}{M_{W}}\right)^{2} \frac{1}{\Lambda^{4}} \sim \frac{10^{-19} A^{2}}{\mathrm{GeV}^{4}}
$$



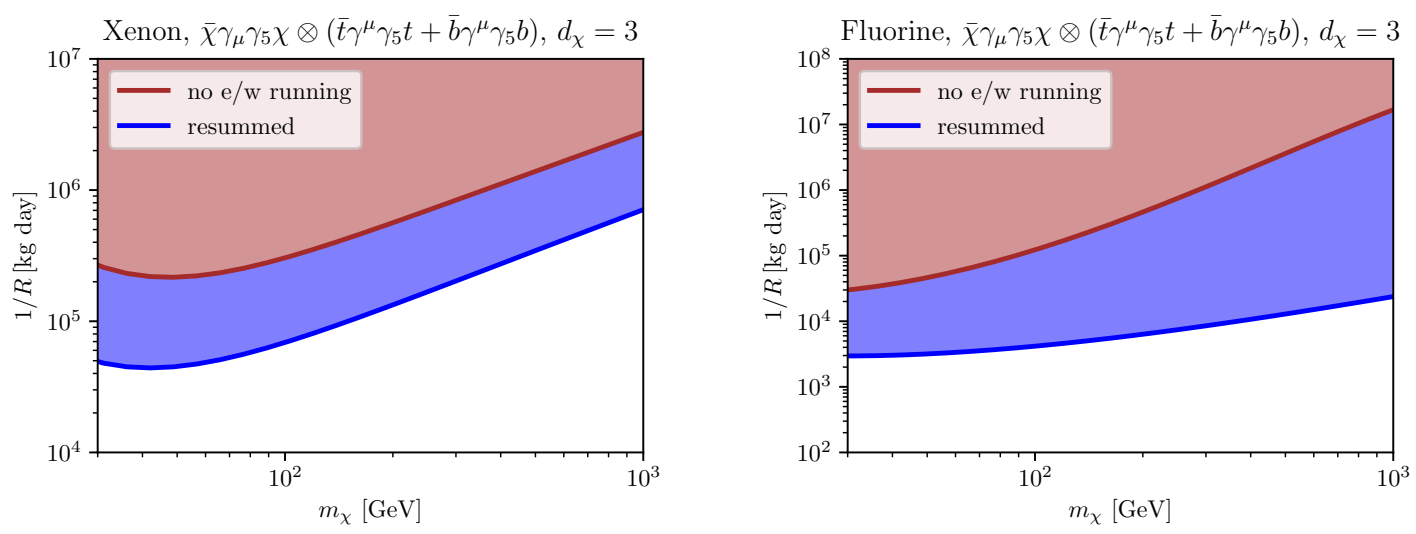

Figure 14. The inverse scattering rates on Xenon (left) and Fluorine (right) for $I_{\chi}=1, Y_{\chi}=0$ Dirac fermion DM with additional dimension-six interactions coupling a DM axial-vector current to the SM axial-vector third generation current, setting $\Lambda=1 \mathrm{TeV}$. The red lines show the predicted rates without mixing; the blue lines after RG resummation. The plots extend in $m_{\chi}$ below the electroweak scale only for illustration purposes - realistic models require extended sets of nontrivial electroweak multiplets that can modify the running (cf. section 2).

In the numerical estimates we assumed a typical momentum transfer of $q=\mathcal{O}(10) \mathrm{MeV}$ and set $\Lambda=1 \mathrm{TeV}$. The first two terms in the square bracket in eq. (5.20) are due to spindependent scattering, with the parametric and numerical estimates shown for Fluorine and ${ }^{129} \mathrm{Xe}$, while they are much smaller for the other main Xenon isotopes. The last term in eq. (5.20) is due to spin-independent scattering. The spin-dependent terms give the dominant contribution to the scattering rates on Fluorine.

The scattering contribution in (5.21) involves QED mixing, converting the third generation quark current to the first generation one, see section 4.3. This contribution is relevant only for scattering on Xenon, where it is, for $\Lambda=1 \mathrm{TeV}$, comparable to the gauge contribution as well as to the spin-dependent scattering in eq. (5.20). Indeed, the left panel of figure 14 shows that the contributions are of the same size.

As already mentioned, the scattering on Fluorine is dominated by the $Q_{18}^{(6)}$-induced contributions, eq. (5.20), where the leading term comes from spin-dependent scattering. Inspection of the $\Sigma^{\prime}, \Sigma^{\prime \prime}$ response functions, ref. [9], shows that spin-dependent scattering on Fluorine is about ten times larger than for ${ }^{129} \mathrm{Xe}$, while the other Xenon isotopes give negligible contributions. In figure 14 we weighted the contributions according to the natural abundance of Xenon isotopes, giving an additional roughly five-fold suppression of the spindependent rate for Xenon. Consequently, the effect of the RG evolution is large only for scattering on fluorine (right panel of figure 14).

\subsubsection{Vector - Axial-vector (first generation)}

Next, we assume that at $\Lambda=1 \mathrm{TeV}$ the only nonzero Wilson coefficients are

$$
-C_{2,1}^{(6)}(\Lambda)=C_{3,1}^{(6)}(\Lambda)=C_{4,1}^{(6)}(\Lambda)
$$

so that the non-renormalizable DM interactions are due to the operator

$$
-Q_{2,1}^{(6)}+Q_{3,1}^{(6)}+Q_{4,1}^{(6)}=\left(\bar{\chi} \gamma_{\mu} \chi\right)\left(\bar{u} \gamma^{\mu} \gamma_{5} u+\bar{d} \gamma^{\mu} \gamma_{5} d\right)
$$



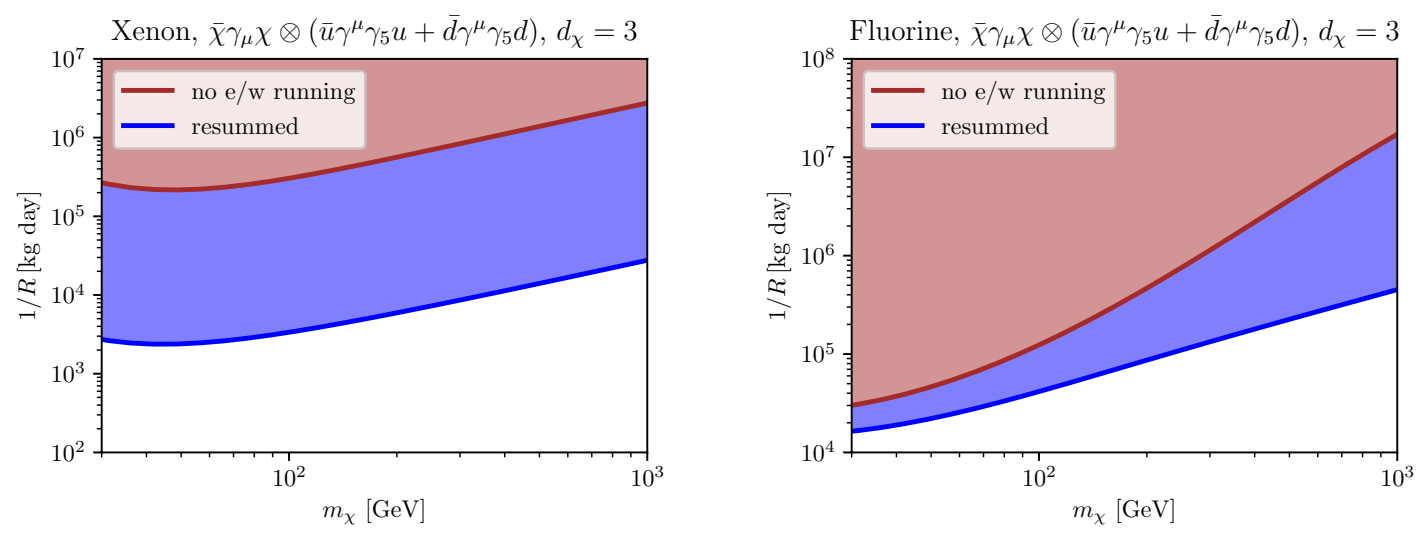

Figure 15. Same as figure 14, but for $I_{\chi}=1, Y_{\chi}=0$ Dirac fermion DM with additional dimensionsix interactions coupling a DM vector current to the SM axial-vector first generation current, setting $\Lambda=1 \mathrm{TeV}$.

This leads to spin-dependent scattering rate that scales roughly as

$$
\frac{d \sigma}{d E_{R}} \propto\left(v_{T}^{\perp}\right)^{2} \frac{1}{\Lambda^{4}} \sim \frac{10^{-18}}{\mathrm{GeV}^{4}},
$$

see section 5. The Higgs penguin contribution, eq. (5.13), dominates over this rate by orders of magnitude. The dominant contribution, however, is mixing induced. The Wilson coefficient $C_{2,1}^{(6)}(\Lambda)$ gets modified by the two-step mixing in the RG evolution to (we neglect numerically subleading contributions)

$$
\begin{aligned}
C_{2,1}^{(6)}\left(M_{W}\right) & =-1-\left(\frac{g_{2}^{2}}{16 \pi^{2}}\right)^{2} \frac{\left[\gamma_{2}^{(0)}\right]_{Q_{2,1}^{(6)}, Q_{5,1}^{(6)}}\left[\gamma_{2}^{(0)}\right]_{Q_{5,1}^{(6)}, Q_{2,1}^{(6)}}}{2} \log ^{2} \frac{M_{W}}{\Lambda} \\
& =-1-\left(\frac{g_{2}^{2}}{16 \pi^{2}}\right)^{2} 36 \log ^{2} \frac{M_{W}}{\Lambda} .
\end{aligned}
$$

The mixing contributions for the other two Wilson coefficients, $C_{3,1}^{(6)}$ and $C_{4,1}^{(6)}$, cancel. This leads to the breaking of the original alignment, eq. (5.22), inducing a coupling to the SM vector current. The product of the large anomalous dimensions and the square of the large $\operatorname{logarithm} \log \left(M_{W} / \Lambda\right)$, together with the coherent enhancement factor, $A^{2}$, leads to

$$
\frac{d \sigma}{d E_{R}} \propto\left(v_{T}^{\perp}\right)^{0} A^{2}\left(\frac{\alpha_{2}^{2}}{(4 \pi)^{2}}\right)^{2}\left(36 \log ^{2} \frac{M_{W}}{\Lambda}\right)^{2} \frac{1}{\Lambda^{4}} \sim \frac{10^{-18} A^{2}}{\mathrm{GeV}^{4}},
$$

resulting in the enhanced scattering rate, as shown in figure 15 .

It is important to realize that it is not sufficient to use the first-order-expanded solution to the RG equations, as the effect arises only at the second order in the mixing. While the effect corresponds to a two-loop correction in the "full theory", our method automatically captures the leading-logarithmic part of it.

\subsubsection{Vector - Axial-vector (third generation)}

Finally, let us consider an initial condition

$$
-C_{2,3}^{(6)}(\Lambda)=C_{3,3}^{(6)}(\Lambda)=C_{4,3}^{(6)}(\Lambda),
$$



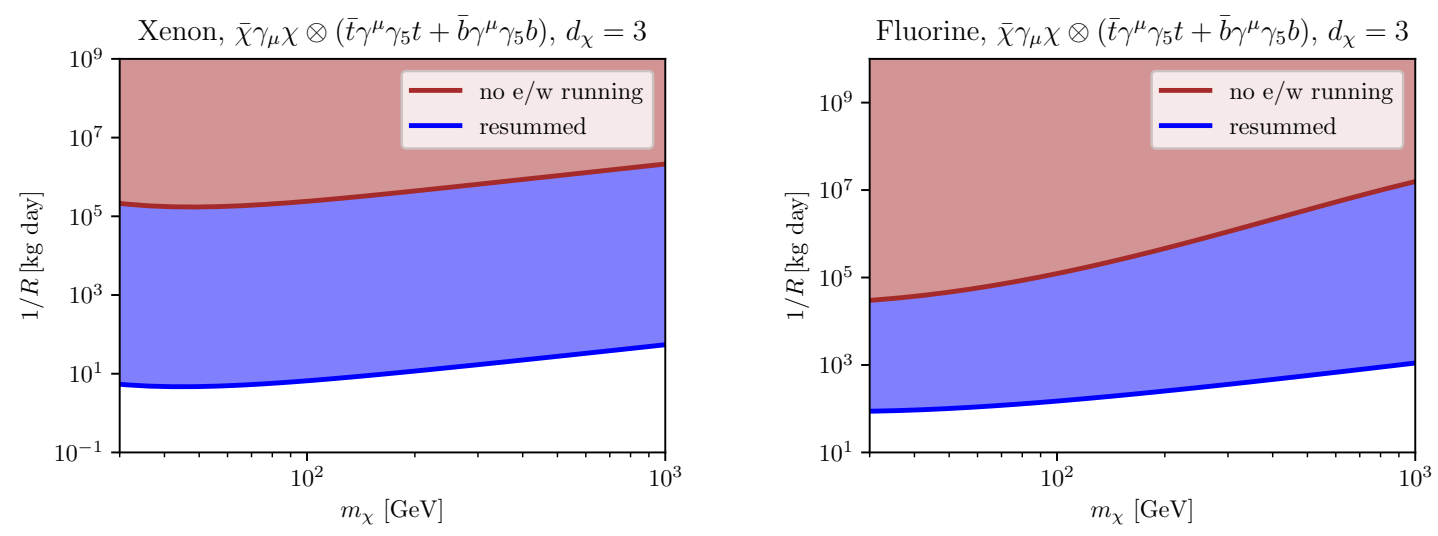

Figure 16. Same as figure 14, but for $I_{\chi}=1, Y_{\chi}=0$ Dirac fermion DM with additional dimension-six interactions coupling a DM vector current to the SM axial-vector third-generation current, setting $\Lambda=1 \mathrm{TeV}$.

so that DM couples to the third generation of quarks through the operator

$$
-Q_{2,3}^{(6)}+Q_{3,3}^{(6)}+Q_{4,3}^{(6)}=\left(\bar{\chi} \gamma_{\mu} \chi\right)\left(\bar{t} \gamma^{\mu} \gamma_{5} t+\bar{b} \gamma^{\mu} \gamma_{5} b\right)
$$

This axial-vector current has a vanishingly small nuclear matrix element, see section 5.1. Without mixing, the leading contribution to the scattering rate is thus due to the renormalizable gauge interactions, eqs. (5.13) and (5.14).

The largest contribution comes, however, from the mixing. At one loop the top-quark Yukawa interactions induce mixing of $Q_{2,3}^{(6)}$ and $Q_{3,3}^{(6)}$ into $Q_{16}^{(6)}$ with anomalous dimensions 6 and -6 , respectively, see eq. (3.40). The contributions add up for the axial-vector quark current, giving, for the initial condition (5.27),

$$
\begin{aligned}
C_{16}^{(6)}\left(M_{W}\right) & =\frac{y_{t}^{2}}{16 \pi^{2}}\left(-\left[\gamma_{t}^{(0)}\right]_{Q_{2,3}^{(6)}, Q_{16}^{(6)}}+\left[\gamma_{t}^{(0)}\right]_{Q_{3,3}^{(6)}, Q_{16}^{(6)}}\right) \log \frac{M_{W}}{\Lambda} \\
& =-12 \frac{y_{t}^{2}}{16 \pi^{2}} \log \frac{M_{W}}{\Lambda} .
\end{aligned}
$$

The above result takes into account the cancelations of contributions due to the actual values of the anomalous dimensions, and neglects numerically subleading terms. (The twostep mixing effect, described in the previous subsection, is still present, but subleading.)

The operator $Q_{16}^{(6)}$ leads to a vector-vector interaction after integrating out the $Z$ boson, cf. eq. (4.16), giving a coherently enhanced scattering cross section of parametric size

$$
\frac{d \sigma}{d E_{R}} \propto\left(v_{T}^{\perp}\right)^{0} A^{2}\left(\frac{\alpha_{t}}{4 \pi}\right)^{2}\left(12 \log \frac{M_{W}}{\Lambda}\right)^{2} \frac{1}{\Lambda^{4}} \sim \frac{10^{-14} A^{2}}{\mathrm{GeV}^{4}} .
$$

which is several orders of magnitude larger than the Higgs-penguin induced one. This is illustrated in figure 15. Included in the numerics is the additional enhancement of the cross section by the resummation of leading QCD logarithms below the weak scale (see ref. [25] for details). Note that the mixing induced effect, eq. (5.30), is independent of the weak isospin of DM and is present even for SM-singlet DM [60]. 


\section{Summary and conclusions}

In this article we presented a Renormalization Group (RG) analysis of Dark Matter (DM) interactions with the SM mediated by higher dimension operators, up to and including dimension six. We calculated the one-loop RG evolution of these operators, for the case of Dirac fermion DM, from the high scale $\Lambda$ down to the weak scale, and the matching to the tower of effective theories below the weak scale, distinguishing the two cases, $m_{\chi} \sim m_{Z}$ and $m_{\chi} \ll m_{Z}$. We allow for DM to be part of an electroweak multiplet.

The loop corrections are important whenever both the renormalizable interactions and the tree-level insertions of higher dimension operators give suppressed direct detection scattering rates. For DM charged under the electroweak gauge group, the scattering due to renormalizable interactions is either spin-dependent or effectively of two-loop size. This means that the contributions from higher dimension interactions, even if loop suppressed, can still give the leading contribution. This is true in particular if the tree-level contributions from higher dimension operators have small nuclear matrix elements, while the loop-induced ones do not. In section 5.2 we illustrated this for three examples of DM coupling to axial-vector quark currents, where the loop-induced effects are especially large. Since the anomalous dimensions are numerically large, the mixing induced effects can dominate the scattering rate even if they are effectively of two-loop order. The RG evolution automatically picks up the leading-logarithmic parts of such corrections to all orders.

The computed corrections are not optional, as they arise from SM particles running in the loop. They thus need to be included when connecting the processes that occur at the mass of the DM (such as the indirect detection and the LHC searches) with the processes occurring at the low scale, e.g., direct detection scattering. The anomalous dimensions are of two types: (i) the contributions due to Higgs exchanges, which are present even in the case that DM is an electroweak singlet, and (ii) the contributions that are due to the exchanges of gauge bosons. The latter are present only if DM is part of an electroweak multiplet.

The resulting RG evolution is implemented in the public code DirectDM [23] and is available at

https://directdm.github.io.

The code should make it relatively straightforward to use our results when comparing indirect detection and LHC bounds with the results of direct detection experiments, including the scattering on electrons, that is in many cases generated already at one-loop level.

There are several directions for future work. The remaining case for Dirac fermion DM, $m_{\chi} \gg m_{Z}$, requires the transition to Heavy DM EFT already above the weak scale. This will result both in a different basis of EFT operators above the electroweak scale, as well as changes to the anomalous dimensions. The calculations of anomalous dimensions should also be extended to include dimension seven operators (the full basis was presented in ref. [26]). Phenomenologically interesting is an extension of our work to several multiplets, which would cover, for instance, bino-wino-higgsino mixing in the MSSM. There are also higher-loop contributions that the leading-logarithmic RG resummation misses. For instance, at two-loop level there is mixing from dimension-six operators with quarks and 
leptons into dipole operators. Such contributions may be important when estimating the dipole contributions to the dark matter scattering rates.

\section{Acknowledgments}

We thank F. D'Eramo and E. Stamou for useful discussions. JZ acknowledges support in part by the DOE grant DE-SC0011784, and thanks the CERN theory group for hospitality for the duration of his sabbatical stay, during which part of this project was worked out. This work was also performed in part at Aspen Center for Physics, which is supported by National Science Foundation grant PHY-1607611. This work was partially supported by a grant from the Simons Foundation. JB and JZ thank the Galileo Galilei Institute for Theoretical Physics for hospitality and the INFN for partial support during the completion of this work.

\section{A Conventions and input}

\section{A.1 Standard model in the unbroken and broken phases}

Here we collect the conventions that we use in the paper. Our convention for the Lorentz vectors is $p^{\mu}=\left(p^{0}, \vec{p}\right), p_{\mu}=\left(p^{0},-\vec{p}\right)$, while for the completely antisymmetric Levi-Civita tensor $\epsilon^{\mu \nu \rho \sigma}$ we use the convention $\epsilon^{0123}=+1$. The field-strength tensors are

$$
\begin{aligned}
G_{\mu \nu}^{a} & =\partial_{\mu} G_{\nu}^{a}-\partial_{\nu} G_{\mu}^{a}-g_{s} f^{a b c} G_{\mu}^{b} G_{\nu}^{c}, \\
W_{\mu \nu}^{a} & =\partial_{\mu} W_{\nu}^{a}-\partial_{\nu} W_{\mu}^{a}+g_{2} \epsilon^{a b c} W_{\mu}^{b} W_{\nu}^{c}, \\
B_{\mu \nu} & =\partial_{\mu} B_{\nu}-\partial_{\nu} B_{\mu} .
\end{aligned}
$$

The $\mathrm{SU}(3), \mathrm{SU}(2)$, and $\mathrm{U}(1)$ coupling constants are $g_{s}, g_{2}$, and $g_{1}$, while $f^{a b c}, \epsilon^{a b c}$ are the completely antisymmetric $\mathrm{SU}(3)$ and $\mathrm{SU}(2)$ structure constants, respectively.

The covariant derivative acting on a fermion $f$ is, in our convention,

$$
D_{\mu} f=\left(\partial_{\mu}-i g_{s} T^{a} G_{\mu}^{a}-i g_{2} \tilde{\tau}^{a} W_{\mu}^{a}+i g_{1} \frac{Y_{f}}{2} B_{\mu}\right) f,
$$

with $T^{a}, \tilde{\tau}^{a}$ the generators of $\mathrm{SU}(3)$ and $\mathrm{SU}(2)$, respectively, and $Y_{f}$ the hypercharge of fermion $f$. Specializing to the DM fields we thus have

$$
D_{\mu} \chi=\left(\partial_{\mu}-i g_{2} \tilde{\tau}^{a} W_{\mu}^{a}+i g_{1} \frac{Y_{\chi}}{2} B_{\mu}\right) \chi,
$$

with $Y_{\chi}$ the DM hypercharge. The $\mathrm{SU}(2)$ generators $\tilde{\tau}^{a}$ for a general representation of weak isospin $I_{\chi}$ can be chosen as

$$
\left(\tilde{\tau}^{1} \pm i \tilde{\tau}^{2}\right)_{k l}=\delta_{k, l \pm 1} \sqrt{\left(I_{\chi} \mp l\right)\left(I_{\chi} \pm l+1\right)}, \quad\left(\tilde{\tau}^{3}\right)_{k l}=l \delta_{k, l},
$$

with $k, l$ running over the values $-I_{\chi},-I_{\chi}+1, \ldots, I_{\chi}-1, I_{\chi}$.

The Higgs Lagrangian in terms of the complex Higgs doublet $H$ reads

$$
\mathcal{L}_{H}=\left(D_{\mu} H\right)^{\dagger} D^{\mu} H-\frac{\lambda}{4}\left(H^{\dagger} H\right)^{2}+\mu^{2} H^{\dagger} H .
$$


In the calculation of the anomalous dimensions above the electroweak scale the Higgs mass term can be neglected as it does not affect the UV divergences.

The Yukawa interactions are given by

$$
\mathcal{L}_{Y}=-\sum_{k, l} \bar{Q}_{L}^{k} Y_{k l}^{u} u_{R}^{l} \tilde{H}-\sum_{k, l} \bar{Q}_{L}^{k} Y_{k l}^{d} d_{R}^{l} H-\sum_{k, l} \bar{L}_{L}^{k} Y_{k l}^{\ell} \ell_{R}^{l} H+\text { h.c. }
$$

with $k, l$ the generation indices, while the charge-conjugated Higgs field is given by $\tilde{H}=$ $i \sigma^{2} H^{*}$. In the calculation of the electroweak mixing we neglect the up, down, strange, electron, and muon Yukawa couplings.

We further complement the Lagrangian involving the matter fields with a gauge-fixing term. It is most convenient to perform the calculation in a background-field gauge; the gauge-fixing Lagrangian can be taken in analogy to the case of QCD $[99,100]$. We use a generalized $R_{\xi}$ gauge, with gauge fixing term [101]

$$
\mathcal{L}_{\mathrm{gf}}=-\frac{1}{2 \xi^{W}}\left[\left(\delta^{a c} \partial_{\mu}+g_{2} \epsilon^{a b c} \hat{W}_{\mu}^{b}\right) W^{c, \mu}\right]^{2}-\frac{1}{2 \xi^{B}}\left(\partial_{\mu} B^{\mu}\right)^{2},
$$

and checked explicitly the $\xi^{W}$ and $\xi^{B}$ gauge-parameter independence of our results.

After electroweak symmetry breaking we use the mass eigenbasis for the gauge bosons,

$$
W_{\mu}^{ \pm}=\left(W_{\mu}^{1} \mp i W_{\mu}^{2}\right) / \sqrt{2}, \quad\left(\begin{array}{c}
Z_{\mu} \\
A_{\mu}
\end{array}\right)=\left(\begin{array}{cc}
c_{w} & s_{w} \\
-s_{w} & c_{w}
\end{array}\right)\left(\begin{array}{c}
W_{\mu}^{3} \\
B_{\mu}
\end{array}\right),
$$

where $c_{w} \equiv \cos \theta_{w}=g_{2} / \sqrt{g_{1}^{2}+g_{2}^{2}}, s_{w} \equiv \sin \theta_{w}$. The electric charge is given by

$$
e=\frac{g_{1} g_{2}}{\sqrt{g_{1}^{2}+g_{2}^{2}}}=g_{2} s_{w}=g_{1} c_{w} .
$$

The electric charge of the components of the DM multiplet is given by the Gell-Mann Nishijima relation, $Q_{\chi}=\tilde{\tau}^{3}+Y_{\chi} / 2$. Defining $\tilde{\tau}^{ \pm}=\tilde{\tau}^{1} \pm i \tilde{\tau}^{2}$, we can write the covariant derivative (A.5) in terms of the broken fields as

$$
D_{\mu}=\partial_{\mu}+i g_{s} T^{a} G_{\mu}^{a}-\frac{i}{\sqrt{2}} \frac{e}{s_{w}}\left(\tilde{\tau}^{-} W_{\mu}^{+}+\tilde{\tau}^{+} W_{\mu}^{-}\right)+i e Q_{\chi} A_{\mu}-\frac{i e}{s_{w} c_{w}}\left(\tilde{\tau}^{3}-s_{w}^{2} Q_{\chi}\right) Z_{\mu} .
$$

The Higgs doublet field after EWSB is given by

$$
H(x)=\left(\begin{array}{c}
G^{+}(x) \\
\frac{1}{\sqrt{2}}\left(v+h(x)+i G^{0}(x)\right)
\end{array}\right),
$$

where $G^{+}(x)$ and $G^{0}(x)$ are the pseudo-Goldstone fields.

\section{A.2 Numerical inputs for the electroweak running}

The parameters used in our numerics for the electroweak RG evolution are $\hat{g}_{1}, \hat{g}_{2}, \hat{g}_{3}, \hat{y}_{c}, \hat{y}_{\tau}$, $\hat{y}_{b}, \hat{y}_{t}$, where the hat denotes the values in the $\overline{\mathrm{MS}}$ scheme at scale $M_{Z}$. All the numerical inputs are taken from ref. [102]. Our strategy to determine the initial values at scale 
$\mu=M_{Z}$ is as follows. We use the values $\sin ^{2} \theta_{w}\left(M_{Z}\right)=0.23122(4), \alpha^{-1}\left(M_{Z}\right)=127.955(10)$ to determine $\hat{g}_{1}$ and $\hat{g}_{2}$ directly via the relation

$$
\sin ^{2} \theta_{w}(\mu) \equiv \frac{g_{1}^{2}(\mu)}{g_{1}^{2}(\mu)+g_{2}^{2}(\mu)} .
$$

In this way we find $\hat{g}_{1}=0.36, \hat{g}_{2}=0.65$. The strong coupling $\hat{g}_{3}=1.22$ is determined from $\alpha_{s}\left(M_{Z}\right)=0.1181(11)$.

To determine $\hat{y}_{\tau}$ we use $m_{\tau}=1.77686(12) \mathrm{GeV}$, and the relations

$$
y_{\tau}=\frac{\sqrt{2} m_{\tau}}{v_{\mathrm{EW}}}, \quad G_{F}=\frac{1}{\sqrt{2} v_{\mathrm{EW}}^{2}} .
$$

Note that $G_{F}=1.1663787(6) \times 10^{-5} \mathrm{GeV}^{-2}$ is $\mathrm{RG}$ invariant, and we neglect the QED running of $m_{\tau}$. We find $\hat{y}_{\tau}\left(M_{Z}\right)=0.010$. We obtain $m_{c}\left(M_{Z}\right)$ by QCD running from $m_{c}\left(m_{c}\right)=1.275(3) \mathrm{GeV}$ and then convert to find $\hat{y}_{c}\left(M_{Z}\right)=0.0045$. We obtain $m_{b}\left(M_{Z}\right)$ by QCD running from $m_{b}\left(m_{b}\right)=4.18_{-0.03}^{+0.04} \mathrm{GeV}$ and then convert to find $\hat{y}_{b}\left(M_{Z}\right)=0.018$. We obtain $m_{t}\left(M_{Z}\right)$ by converting the top-quark pole mass $M_{t}=173.0(0.4) \mathrm{GeV}$ to the QCD and electroweak $\overline{M S}$ scheme at scale $\mu=M_{t}$, and use subsequent QCD and electroweak running from $\mu=M_{t}$ to $\mu=M_{Z}$. We find $\hat{y}_{t}\left(M_{Z}\right)=1.05$. For the Higgs self-coupling $\lambda$, we take $\lambda\left(M_{Z}\right) \sim \lambda\left(M_{h}\right)=2 \sqrt{2} M_{h}^{2} G_{F} \approx 0.52$ with $M_{h}=125.1 \mathrm{GeV}$.

\section{B Nonrelativistic coefficients}

The operators $\mathcal{Q}_{23, q}^{(7,0)}$ and $\mathcal{Q}_{25}^{(7,0)}$, defined in eq. (4.47), lead to the following additional contributions to the nonrelativistic coefficients:

$$
c_{1}^{p}=\frac{3}{4} m_{p}\left(\hat{\mathcal{C}}_{25}^{(7)} f_{g, p}^{(2)}+\sum_{q} \hat{\mathcal{C}}_{23, q}^{(7)} f_{q, p}^{(2)}\right),
$$

with $f_{u, p}^{(2)}=0.346(7), f_{d, p}^{(2)}=0.192(6), f_{s, p}^{(2)}=0.034(3)$, and $f_{g, p}^{(2)}=0.419(19)$, evaluated at renormalizations scale $\mu=2 \mathrm{GeV}$ [16]. The coefficients for neutrons can be obtained by the exchange $p \leftrightarrow n, u \leftrightarrow d$.

\section{SM EFT operators}

In this appendix we provide the results for the mixing of the SM-DM operators into the pure SM operators, restricting the discussion to the SM operators that enter the RG running at one loop. Assuming conservation of lepton and baryon number, only dimension-six operators are relevant. The dimension-six effective Lagrangian is (we use the basis in ref. [103], but with renamed operators)

$$
\mathcal{L}=\sum_{a} \frac{C_{a}^{\mathrm{SM},(6)}}{\Lambda^{2}} S_{a}^{(6)},
$$


where the operators involving only quark fields are

$$
\begin{array}{rlrl}
S_{1, i j}^{(6)} & =\left(\bar{Q}_{L}^{i} \gamma_{\mu} \tau^{a} Q_{L}^{i}\right)\left(\bar{Q}_{L}^{j} \gamma^{\mu} \tau^{a} Q_{L}^{j}\right), & S_{2, i j}^{(6)} & =\left(\bar{Q}_{L}^{i} \gamma_{\mu} Q_{L}^{i}\right)\left(\bar{Q}_{L}^{j} \gamma^{\mu} Q_{L}^{j}\right), \\
S_{3, i j}^{(6)} & =\left(\bar{Q}_{L}^{i} \gamma_{\mu} Q_{L}^{i}\right)\left(\bar{u}_{R}^{j} \gamma^{\mu} u_{R}^{j}\right), & S_{4, i j}^{(6)} & =\left(\bar{Q}_{L}^{i} \gamma_{\mu} Q_{L}^{i}\right)\left(\bar{d}_{R}^{j} \gamma^{\mu} d_{R}^{j}\right), \\
S_{5, i j}^{(6)} & =\left(\bar{u}_{R}^{i} \gamma_{\mu} u_{R}^{i}\right)\left(\bar{u}_{R}^{j} \gamma^{\mu} u_{R}^{j}\right), & S_{6, i j}^{(6)} & =\left(\bar{u}_{R}^{i} \gamma_{\mu} u_{R}^{i}\right)\left(\bar{d}_{R}^{j} \gamma^{\mu} d_{R}^{j}\right), \\
S_{7, i j}^{(6)} & =\left(\bar{d}_{R}^{i} \gamma_{\mu} d_{R}^{i}\right)\left(\bar{d}_{R}^{j} \gamma^{\mu} d_{R}^{j}\right) . &
\end{array}
$$

The operators involving only lepton fields can be chosen as

$$
\begin{aligned}
S_{8, i j}^{(6)} & =\left(\bar{L}_{L}^{i} \gamma_{\mu} L_{L}^{i}\right)\left(\bar{L}_{L}^{j} \gamma^{\mu} L_{L}^{j}\right), & S_{9, i j}^{(6)}=\left(\bar{L}_{L}^{i} \gamma_{\mu} L_{L}^{i}\right)\left(\bar{\ell}_{R}^{j} \gamma^{\mu} \ell_{R}^{j}\right), \\
S_{10, i j}^{(6)} & =\left(\bar{\ell}_{R}^{i} \gamma_{\mu} \ell_{R}^{i}\right)\left(\bar{\ell}_{R}^{j} \gamma^{\mu} \ell_{R}^{j}\right) . &
\end{aligned}
$$

The mixed quark-lepton operators are

$$
\begin{array}{rlrl}
S_{11, i j}^{(6)} & =\left(\bar{Q}_{L}^{i} \gamma_{\mu} \tau^{a} Q_{L}^{i}\right)\left(\bar{L}_{L}^{j} \gamma^{\mu} \tau^{a} L_{L}^{j}\right), & S_{12, i j}^{(6)} & =\left(\bar{Q}_{L}^{i} \gamma_{\mu} Q_{L}^{i}\right)\left(\bar{L}_{L}^{j} \gamma^{\mu} L_{L}^{j}\right), \\
S_{13, i j}^{(6)} & =\left(\bar{Q}_{L}^{i} \gamma_{\mu} Q_{L}^{i}\right)\left(\bar{\ell}_{R}^{j} \gamma^{\mu} \ell_{R}^{j}\right), & S_{14, i j}^{(6)}=\left(\bar{u}_{R}^{i} \gamma^{\mu} u_{R}^{i}\right)\left(\bar{L}_{L}^{j} \gamma_{\mu} L_{L}^{j}\right), \\
S_{15, i j}^{(6)}=\left(\bar{d}_{R}^{i} \gamma^{\mu} d_{R}^{i}\right)\left(\bar{L}_{L}^{j} \gamma_{\mu} L_{L}^{j}\right), & S_{16, i j}^{(6)}=\left(\bar{u}_{R}^{i} \gamma^{\mu} u_{R}^{i}\right)\left(\bar{\ell}_{R}^{j} \gamma_{\mu} \ell_{R}^{j}\right), \\
S_{17, i j}^{(6)}=\left(\bar{d}_{R}^{i} \gamma^{\mu} d_{R}^{i}\right)\left(\bar{\ell}_{R}^{j} \gamma_{\mu} \ell_{R}^{j}\right), &
\end{array}
$$

while the Higgs-fermion operators can be taken as

$$
\begin{aligned}
& S_{18, i}^{(6)}=\left(\bar{Q}_{L}^{i} \gamma^{\mu} \tau^{a} Q_{L}^{i}\right)\left(H^{\dagger} i \stackrel{\leftrightarrow}{D_{\mu}^{a}} H\right), \quad S_{19, i}^{(6)}=\left(\bar{Q}_{L}^{i} \gamma^{\mu} Q_{L}^{i}\right)\left(H^{\dagger} i \stackrel{\leftrightarrow}{D} \mu H\right), \\
& S_{20, i}^{(6)}=\left(\bar{u}_{R}^{i} \gamma^{\mu} u_{R}^{i}\right)\left(H^{\dagger} i \stackrel{\leftrightarrow}{D}_{\mu} H\right), \quad S_{21, i}^{(6)}=\left(\bar{d}_{R}^{i} \gamma^{\mu} d_{R}^{i}\right)\left(H^{\dagger} i \stackrel{\leftrightarrow}{D}_{\mu} H\right), \\
& S_{22, i}^{(6)}=\left(\bar{L}_{L}^{i} \gamma^{\mu} \tau^{a} L_{L}^{i}\right)\left(H^{\dagger} i \stackrel{\leftrightarrow}{D_{\mu}^{a}} H\right), \quad S_{23, i}^{(6)}=\left(\bar{L}_{L}^{i} \gamma^{\mu} L_{L}^{i}\right)\left(H^{\dagger} i \stackrel{\leftrightarrow}{D_{\mu}} H\right) \\
& S_{24, i}^{(6)}=\left(\bar{\ell}_{R}^{i} \gamma^{\mu} \ell_{R}^{i}\right)\left(H^{\dagger} i \stackrel{\leftrightarrow}{D} \mu H\right)
\end{aligned}
$$

The remaining operator, involving only Higgs fields, is

$$
S_{25}^{(6)}=\left(H^{\dagger} i \stackrel{\leftrightarrow}{D}_{\mu} H\right)\left(H^{\dagger} i \stackrel{\leftrightarrow}{D}^{\mu} H\right)
$$

The mixing of the SM sector into the DM-SM sector proceeds via penguin insertions. The nonzero results for the four-fermion operators are, for $i=j$,

$$
\left[\gamma_{1}^{(0)}\right]_{S_{1 \ldots 7, i}^{(6)} \times Q_{2 \ldots 4, i}^{(6)}}=Y_{\chi}\left(\begin{array}{ccc}
\frac{1}{6} & 0 & 0 \\
\frac{14}{9} & 0 & 0 \\
\frac{4}{3} & \frac{2}{3} & 0 \\
-\frac{2}{3} & 0 & \frac{2}{3} \\
0 & \frac{32}{9} & 0 \\
0 & -\frac{2}{3} & \frac{4}{3} \\
0 & 0 & -\frac{16}{9}
\end{array}\right), \quad\left[\gamma_{1}^{(0)}\right]_{S_{12 \ldots 17, i i}^{(6)} \times Q_{2 \ldots 4, i}^{(6)}}=Y_{\chi}\left(\begin{array}{ccc}
-\frac{2}{3} & 0 & 0 \\
-\frac{2}{3} & 0 & 0 \\
0 & -\frac{2}{3} & 0 \\
0 & 0 & -\frac{2}{3} \\
0 & -\frac{2}{3} & 0 \\
0 & 0 & -\frac{2}{3}
\end{array}\right) \text {, }
$$


for mixing into operators with quark currents, and

$$
\left[\gamma_{1}^{(0)}\right]_{S_{8 \ldots 10, i i}^{(6)} \times Q_{10,11, i}^{(6)}}=Y_{\chi}\left(\begin{array}{cc}
-2 & 0 \\
-\frac{2}{3} & -\frac{2}{3} \\
0 & -\frac{8}{3}
\end{array}\right), \quad\left[\gamma_{1}^{(0)}\right]_{S_{12 \ldots 17, i i}^{(6)} \times Q_{10,11, i}^{(6)}}=Y_{\chi}\left(\begin{array}{cc}
\frac{2}{3} & 0 \\
0 & \frac{2}{3} \\
\frac{4}{3} & 0 \\
-\frac{2}{3} & 0 \\
0 & \frac{4}{3} \\
0 & -\frac{2}{3}
\end{array}\right) .
$$

for mixing into operators with lepton currents.

The mixing proportional to $g_{2}$ has only a few non-vanishing entries, given by

$$
\begin{array}{rlrl}
{\left[\gamma_{2}^{(0)}\right]_{S_{1, i i}^{(6)} Q_{1, i}^{(6)}}} & =\frac{10}{3}, & {\left[\gamma_{2}^{(0)}\right]_{S_{2, i i}^{(6)} Q_{1, i}^{(6)}}} & =\left[\gamma_{2}^{(0)}\right]_{S_{8, i i}^{(6)} Q_{9, i}^{(6)}}=\frac{8}{3}, \\
{\left[\gamma_{2}^{(0)}\right]_{S_{11, i i}^{(6)} Q_{1, i}^{(6)}}} & =\frac{2}{3}, \quad\left[\gamma_{2}^{(0)}\right]_{S_{11, i i}^{(6)} Q_{9, i}^{(6)}}=2 .
\end{array}
$$

All the other entries are zero.

The result for $i \neq j$ are (note that the order of the flavor indices matters, except when the operator is symmetric in $i$ and $j$ )

$$
\begin{aligned}
& {\left[\gamma_{1}^{(0)}\right]_{S_{2 \ldots 4, i j}^{(6)} \times Q_{2, i}^{(6)}} }=Y_{\chi}\left(\frac{2}{3}, \frac{4}{3},-\frac{2}{3}\right), \\
& {\left[\gamma_{1}^{(0)}\right]_{S_{8 \ldots 10, i j}^{(6)} \times Q_{10 \ldots 11, i}^{(6)}}=\left[\gamma_{1}^{(0)}\right]_{S_{12 \ldots 14, i j}^{(6)} \times Q_{2 \ldots, i}^{(6)}}=Y_{\chi}\left(\begin{array}{cc}
-\frac{2}{3} & 0 \\
-\frac{2}{3} & 0 \\
0 & -\frac{2}{3}
\end{array}\right), }
\end{aligned}
$$

as well as

$$
\begin{aligned}
{\left[\gamma_{1}^{(0)}\right]_{S_{5 \ldots 7, i j}^{(6)} \times Q_{3 \ldots 4, i}^{(6)}}=Y_{\chi}\left(\begin{array}{cc}
\frac{4}{3} & 0 \\
-\frac{2}{3} & 0 \\
0 & -\frac{2}{3}
\end{array}\right), } & {\left[\gamma_{1}^{(0)}\right]_{S_{15 \ldots 17, i j}^{(6)} \times Q_{3 \ldots 4, i}^{(6)}}=Y_{\chi}\left(\begin{array}{cc}
0 & -\frac{2}{3} \\
-\frac{2}{3} & 0 \\
0 & -\frac{2}{3}
\end{array}\right) } \\
{\left[\gamma_{1}^{(0)}\right]_{S_{3 \ldots \ldots, j i}^{(6)} \times Q_{3 \ldots 4, i}^{(6)}}=Y_{\chi}\left(\begin{array}{cc}
\frac{2}{3} & 0 \\
0 & \frac{2}{3} \\
\frac{4}{3} & 0 \\
0 & \frac{4}{3}
\end{array}\right), } & {\left[\gamma_{1}^{(0)}\right]_{S_{14 \ldots 17, j i}^{(6)} \times Q_{10 \ldots 11, i}^{(6)}}=Y_{\chi}\left(\begin{array}{cc}
\frac{4}{3} & 0 \\
-\frac{2}{3} & 0 \\
0 & \frac{4}{3} \\
0 & -\frac{2}{3}
\end{array}\right), }
\end{aligned}
$$

and

$$
\begin{aligned}
{\left[\gamma_{1}^{(0)}\right]_{S_{9, j i}^{(6)} \times Q_{11, i}^{(6)}} } & =-\frac{2 Y_{\chi}}{3}, & {\left[\gamma_{1}^{(0)}\right]_{S_{12 \ldots 13, j i}^{(6)} \times Q_{10 \ldots 11, i}^{(6)}} } & =Y_{\chi} \operatorname{diag}\left(\frac{2}{3}, \frac{2}{3}\right), \\
{\left[\gamma_{2}^{(0)}\right]_{S_{1, i j}^{(6)} Q_{1, i}^{(6)}} } & =\left[\gamma_{2}^{(0)}\right]_{S_{11, j i}^{(6)} Q_{9, i}^{(6)}}=2, & {\left[\gamma_{2}^{(0)}\right]_{S_{11, i j}^{(6)} Q_{1, i}^{(6)}} } & =\frac{2}{3} .
\end{aligned}
$$

All the other entries are zero.

The nonzero mixings of the operators involving Higgs currents are given by

$$
\begin{array}{lll}
{\left[\gamma_{2}^{(0)}\right]_{S_{18, i}^{(6)} Q_{1, i}^{(6)}}=\left[\gamma_{2}^{(0)}\right]_{S_{22, i}^{(6)} Q_{9, i}^{(6)}}=\frac{1}{3},} & {\left[\gamma_{2}^{(0)}\right]_{S_{18, i}^{(6)} Q_{15}^{(6)}}=2,} \\
{\left[\gamma_{2}^{(0)}\right]_{S_{22, i}^{(6)} Q_{15}^{(6)}}=\frac{2}{3},} & {\left[\gamma_{2}^{(0)}\right]_{S_{25}^{(6)} Q_{15}^{(6)}}=\frac{2}{3},}
\end{array}
$$


as well as by

$$
\begin{aligned}
{\left[\gamma_{1}^{(0)}\right]_{S_{19, i}^{(6)} \cdots S_{21, i}^{(6)} \times Q_{2, i}^{(6)} \cdots Q_{4, i}^{(6)}} } & =\frac{1}{3} Y_{\chi} \operatorname{diag}(1,1,1), \\
{\left[\gamma_{1}^{(0)}\right]_{S_{23, i}^{(6)}, S_{24, i}^{(6)} \times Q_{10, i}^{(6)}, Q_{11, i}^{(6)}} } & =\frac{1}{3} Y_{\chi} \operatorname{diag}(1,1), \\
{\left[\gamma_{2}^{(0)}\right]_{S_{18, i}^{(6)} Q_{1, i}^{(6)}}=\left[\gamma_{2}^{(0)}\right]_{S_{22, i}^{(6)} Q_{9, i}^{(6)}} } & =\frac{1}{3},
\end{aligned}
$$

and

$$
\begin{array}{lll}
{\left[\gamma_{1}^{(0)}\right]_{S_{19, i}^{(6)} Q_{16}^{(6)}}=\frac{2}{3} Y_{\chi}, \quad\left[\gamma_{1}^{(0)}\right]_{S_{20, i}^{(6)} Q_{16}^{(6)}}=\frac{4}{3} Y_{\chi},} & {\left[\gamma_{1}^{(0)}\right]_{S_{21, i}^{(6)} Q_{16}^{(6)}}=-\frac{2}{3} Y_{\chi},} \\
{\left[\gamma_{1}^{(0)}\right]_{S_{23, i}^{(6)} Q_{16}^{(6)}}=\left[\gamma_{1}^{(0)}\right]_{S_{24, i}^{(6)} Q_{16}^{(6)}}=-\frac{2}{3} Y_{\chi},} & {\left[\gamma_{1}^{(0)}\right]_{S_{25}^{(6)} Q_{16}^{(6)}}=\frac{5}{6} Y_{\chi} .}
\end{array}
$$

All the other entries vanish.

The mixing of the DM-SM sector into the SM sector also proceeds only via penguin insertions. The SM four fermion operators in eqs. (C.2)-(C.11) carry two generation indices, where the order of the indices is important. First, we present the anomalous dimensions proportional to $g_{1}^{2}$. The mixing of $Q_{2, i}^{(6)}-Q_{4, i}^{(6)}$ into $S_{2, i j}^{(6)}-S_{7, i j}^{(6)}$ is given by

$$
\begin{aligned}
& {\left[\gamma_{1}^{(0)}\right]_{Q_{2, i}^{(6)}, S_{2, i j}^{(6)}}=\left[\gamma_{1}^{(0)}\right]_{Q_{3, i}^{(6)}, S_{3, j i}^{(6)}}=\left[\gamma_{1}^{(0)}\right]_{Q_{4, i}^{(6)}, S_{4, j i}^{(6)}}=\frac{2}{9} Y_{\chi} d_{\chi},} \\
& {\left[\gamma_{1}^{(0)}\right]_{Q_{2, i}^{(6)}, S_{3, i j}^{(6)}}=\left[\gamma_{1}^{(0)}\right]_{Q_{3, i}^{(6)}, S_{5, i j}^{(6)}}=\left[\gamma_{1}^{(0)}\right]_{Q_{4, i}^{(6)}, S_{6, j i}^{(6)}}=\frac{8}{9} Y_{\chi} d_{\chi},} \\
& {\left[\gamma_{1}^{(0)}\right]_{Q_{2, i}^{(6)}, S_{4, i j}^{(6)}}=\left[\gamma_{1}^{(0)}\right]_{Q_{3, i}^{(6)}, S_{6, i j}^{(6)}}=\left[\gamma_{1}^{(0)}\right]_{Q_{4, i}^{(6)}, S_{7, i j}^{(6)}}=-\frac{4}{9} Y_{\chi} d_{\chi},}
\end{aligned}
$$

while the remaining entries are zero. The mixing of $Q_{5, i}^{(6)}-Q_{18}^{(6)}$ into $S_{1, i j}^{(6)}-S_{7, i j}^{(6)}$ vanishes. The mixing of $Q_{2, i}^{(6)}-Q_{4, i}^{(6)}$ into $S_{12, i j}^{(6)} S_{17, i j}^{(6)}$ is given by

$$
\begin{aligned}
& {\left[\gamma_{1}^{(0)}\right]_{Q_{2, i}^{(6)}, S_{12, i j}^{(6)}}=\left[\gamma_{1}^{(0)}\right]_{Q_{3, i}^{(6)}, S_{14, i j}^{(6)}}=\left[\gamma_{1}^{(0)}\right]_{Q_{4, i}^{(6)}, S_{15, i j}^{(6)}}=-\frac{2}{3} Y \chi d_{\chi},} \\
& {\left[\gamma_{1}^{(0)}\right]_{Q_{2, i}^{(6)}, S_{13, i j}^{(6)}}=\left[\gamma_{1}^{(0)}\right]_{Q_{3, i}^{(6)}, S_{16, i j}^{(6)}}=\left[\gamma_{1}^{(0)}\right]_{Q_{4, i}^{(6)}, S_{17, i j}^{(6)}}=-\frac{4}{3} Y_{\chi} d_{\chi} .}
\end{aligned}
$$

The mixing of $Q_{1, i}^{(6)}-Q_{4, i}^{(6)}$ into $S_{12, j i}^{(6)} S_{17, j i}^{(6)}$, with reversed indices, vanishes. The mixing of $Q_{1, i}^{(6)}-Q_{4, i}^{(6)}$ into $S_{19, i}^{(6)}{ }^{(6)}(6)$ is given by

$$
\left[\gamma_{1}^{(0)}\right]_{Q_{2, i}^{(6)}, S_{19, i}^{(6)}}=\left[\gamma_{1}^{(0)}\right]_{Q_{3, i}^{(6)}, S_{20, i}^{(6)}}=\left[\gamma_{1}^{(0)}\right]_{Q_{4, i}^{(6)}, S_{21, i}^{(6)}}=\frac{2}{3} Y_{\chi} d_{\chi}
$$

The mixing of $Q_{10, i}^{(6)}, Q_{11, i}^{(6)}$ into the operators $S_{8, i j}^{(6)} S_{10, i j}^{(6)}$ is given by

$$
\begin{aligned}
& {\left[\gamma_{1}^{(0)}\right]_{Q_{10, i}^{(6)}, S_{8, i j}^{(6)}}=\left[\gamma_{1}^{(0)}\right]_{Q_{11, i}^{(6)}, S_{9, j i}^{(6)}}=-\frac{2}{3} Y_{\chi} d_{\chi},} \\
& {\left[\gamma_{1}^{(0)}\right]_{Q_{10, i}^{(6)}, S_{9, i j}^{(6)}}=\left[\gamma_{1}^{(0)}\right]_{Q_{11, i}^{(6)}, S_{10, i j}^{(6)}}=-\frac{4}{3} Y_{\chi} d_{\chi} .}
\end{aligned}
$$


The mixing of $Q_{10, i}^{(6)}, Q_{11, i}^{(6)}$ into the operators $S_{12, i j}^{(6)} S_{17, i j}^{(6)}$ is given by

$$
\begin{aligned}
& {\left[\gamma_{1}^{(0)}\right]_{Q_{10, i}^{(6)}, S_{12, j i}^{(6)}}=\left[\gamma_{1}^{(0)}\right]_{Q_{11, i}^{(6)}, S_{13, j i}^{(6)}}=\frac{2}{9} Y_{\chi} d_{\chi},} \\
& {\left[\gamma_{1}^{(0)}\right]_{Q_{10, i}^{(6)}, S_{14, j i}^{(6)}}=\left[\gamma_{1}^{(0)}\right]_{Q_{11, i}^{(6)}, S_{16, j i}^{(6)}}=\frac{8}{9} Y_{\chi} d_{\chi},} \\
& {\left[\gamma_{1}^{(0)}\right]_{Q_{10, i}^{(6)}, S_{15, j i}^{(6)}}=\left[\gamma_{1}^{(0)}\right]_{Q_{11, i}^{(6)}, S_{17, j i}^{(6)}}=-\frac{4}{9} Y_{\chi} d_{\chi},}
\end{aligned}
$$

whereas the mixing $Q_{10, i}^{(6)}, Q_{11, i}^{(6)}$ into the operators $S_{12, i j}^{(6)} S_{17, i j}^{(6)}$ vanishes. The mixing of $Q_{10, i}^{(6)}, Q_{11, i}^{(6)}$ into the operators $S_{23, i}^{(6)}, S_{24, i}^{(6)}$ is given by

$$
\left[\gamma_{1}^{(0)}\right]_{Q_{10, i}^{(6)} S_{23, i}^{(6)}}=\left[\gamma_{1}^{(0)}\right]_{Q_{11, i}^{(6)} S_{24, i}^{(6)}}=\frac{2}{3} Y_{\chi} d_{\chi}
$$

The mixing of Higgs-DM into SM is given by

$$
\left[\gamma_{1}^{(0)}\right]_{Q_{16, i}^{(6)} \times S_{19, i}^{(6)} \cdots S_{25, i}^{(6)}}=Y_{\chi} d_{\chi}\left(\frac{2}{9} \frac{8}{9}-\frac{4}{9} 0-\frac{2}{3}-\frac{4}{3} \frac{2}{3}\right) .
$$

The mixing proportional to $g_{2}$ has only a few non-vanishing entries, given by

$$
\begin{aligned}
{\left[\gamma_{2}^{(0)}\right]_{Q_{1, i}^{(6)} S_{1, i j}^{(6)}} } & =\left[\gamma_{2}^{(0)}\right]_{Q_{1, i}^{(6)} S_{11, i j}^{(6)}}=\left[\gamma_{2}^{(0)}\right]_{Q_{1, i}^{(6)} S_{18, i j}^{(6)}}=\left[\gamma_{2}^{(0)}\right]_{Q_{9, i}^{(6)} S_{11, i j}^{(6)}} \\
& =\left[\gamma_{2}^{(0)}\right]_{Q_{9, i}^{(6)} S_{22, i}^{(6)}}=\left[\gamma_{2}^{(0)}\right]_{Q_{15}^{(6)} S_{18, i}^{(6)}}=\left[\gamma_{2}^{(0)}\right]_{Q_{15}^{(6)} S_{22, i}^{(6)}}=\frac{8}{9} \mathcal{J}_{\chi} d_{\chi}, \\
{\left[\gamma_{2}^{(0)}\right]_{Q_{9, i}^{(6)} S_{8, i j}^{(6)}} } & =\left[\gamma_{2}^{(0)}\right]_{Q_{15}^{(6)} S_{25}^{(6)}}=\frac{2}{9} \mathcal{J}_{\chi} d_{\chi} .
\end{aligned}
$$

Again, all the undisplayed entries vanish. The mixing of the SM operators among themselves can be taken from the literature [104-106].

\section{Mixing in the dark sector}

In this appendix we provide the results for the mixing of the operators in the SM-DM sector into the pure DM operators. We write the dimension-six effective Lagrangian as

$$
\mathcal{L}=\sum_{a} \frac{C_{a}^{\mathrm{DM},(6)}}{\Lambda^{2}} S_{a}^{(6)},
$$

where the relevant operators are given in eq. (2.18) (recall that we neglect the mixing of operators within the dark sector).

The mixing of DM-SM operators into DM operators is given by

$$
\begin{gathered}
{\left[\gamma_{1}^{(0)}\right]_{Q_{2 \ldots, i}^{(6)} \times D_{1}^{(6)}}=\left[\gamma_{1}^{(0)}\right]_{Q_{6 \ldots 8, i}^{(6)} \times D_{2}^{(6)}}=Y_{\chi}\left(\begin{array}{c}
\frac{2}{3} \\
\frac{4}{3} \\
-\frac{2}{3}
\end{array}\right),} \\
{\left[\gamma_{1}^{(0)}\right]_{Q_{10,11, i}^{(6)} \times D_{1}^{(6)}}=\left[\gamma_{1}^{(0)}\right]_{Q_{13,14, i}^{(6)} \times D_{2}^{(6)}}=Y_{\chi}\left(\begin{array}{c}
-\frac{2}{3} \\
-\frac{2}{3}
\end{array}\right),}
\end{gathered}
$$




$$
\left[\gamma_{1}^{(0)}\right]_{Q_{16}^{(6)} \times D_{1}^{(6)}}=\left[\gamma_{1}^{(0)}\right]_{Q_{18}^{(6)} \times D_{2}^{(6)}}=\frac{1}{3} Y_{\chi},
$$

and

$$
\begin{aligned}
& {\left[\gamma_{2}^{(0)}\right]_{Q_{1, i}^{(6)} D_{3}^{(6)}}=\left[\gamma_{2}^{(0)}\right]_{Q_{5, i}^{(6)} D_{4}^{(6)}}=2,} \\
& {\left[\gamma_{2}^{(0)}\right]_{Q_{9, i}^{(6)} D_{3}^{(6)}}=\left[\gamma_{2}^{(0)}\right]_{Q_{12, i}^{(6)} D_{4}^{(6)}}=\frac{2}{3},} \\
& {\left[\gamma_{2}^{(0)}\right]_{Q_{15}^{(6)} D_{3}^{(6)}}=\left[\gamma_{2}^{(0)}\right]_{Q_{17}^{(6)} D_{4}^{(6)}}=\frac{1}{3},}
\end{aligned}
$$

while the mixing of the DM operators into the DM-SM sector is given by

$$
\begin{aligned}
& {\left[\gamma_{1}^{(0)}\right]_{D_{1}^{(6)} \ldots D_{4}^{(6)} \times Q_{1, i}^{(6)} \cdots Q_{4, i}^{(6)}}=Y_{\chi}\left(\begin{array}{cccc}
0 & \frac{2}{9}+\frac{4}{9} d_{\chi} & \frac{8}{9}+\frac{16}{9} d_{\chi} & -\frac{4}{9}-\frac{8}{9} d_{\chi} \\
0 & 0 & 0 & 0 \\
0 & \frac{2}{9} \mathcal{J}_{\chi} & \frac{8}{9} \mathcal{J}_{\chi} & -\frac{4}{9} \mathcal{J}_{\chi} \\
0 & 0 & 0 & 0
\end{array}\right),} \\
& {\left[\gamma_{1}^{(0)}\right]_{D_{1}^{(6)} \ldots D_{4}^{(6)} \times Q_{5, i}^{(6)} \cdots Q_{8, i}^{(6)}}=Y_{\chi}\left(\begin{array}{cccc}
0 & 0 & 0 & 0 \\
0 & \frac{2}{9}+\frac{2}{9} d_{\chi} & \frac{8}{9}+\frac{8}{9} d_{\chi} & -\frac{4}{9}-\frac{4}{9} d_{\chi} \\
0 & 0 & 0 & 0 \\
0 & \frac{2}{9} \mathcal{J}_{\chi} & \frac{8}{9} \mathcal{J}_{\chi} & -\frac{4}{9} \mathcal{J}_{\chi}
\end{array}\right)} \\
& {\left[\gamma_{1}^{(0)}\right]_{D_{1}^{(6)} \ldots D_{4}^{(6)} \times Q_{9, i}^{(6)} \cdots Q_{14, i}^{(6)}}=Y_{\chi}\left(\begin{array}{cccccc}
0 & -\frac{2}{3}-\frac{4}{3} d_{\chi}-\frac{4}{3}-\frac{8}{3} d_{\chi} & 0 & 0 & 0 \\
0 & 0 & 0 & 0 & -\frac{2}{3}-\frac{2}{3} d_{\chi}-\frac{4}{3}-\frac{4}{3} d_{\chi} \\
0 & -\frac{2}{3} \mathcal{J}_{\chi} & -\frac{4}{3} \mathcal{J}_{\chi} & 0 & 0 & 0 \\
0 & 0 & 0 & 0 & -\frac{2}{3} \mathcal{J}_{\chi} & -\frac{4}{3} \mathcal{J}_{\chi}
\end{array}\right),} \\
& {\left[\gamma_{1}^{(0)}\right]_{D_{1}^{(6)} \ldots D_{4}^{(6)} \times Q_{15}^{(6)} \cdots Q_{18}^{(6)}}=Y_{\chi}\left(\begin{array}{cccc}
0 & \frac{2}{3}+\frac{4}{3} d_{\chi} & 0 & 0 \\
0 & 0 & 0 & \frac{2}{3}+\frac{2}{3} d_{\chi} \\
0 & \frac{2}{3} \mathcal{J}_{\chi} & 0 & 0 \\
0 & 0 & 0 & \frac{2}{3} \mathcal{J}_{\chi}
\end{array}\right)}
\end{aligned}
$$

and

$$
\begin{aligned}
{\left[\gamma_{2}^{(0)}\right]_{D_{1}^{(6)} Q_{1, i}^{(6)}} } & =\left[\gamma_{2}^{(0)}\right]_{D_{2}^{(6)} Q_{5, i}^{(6)}}=\left[\gamma_{2}^{(0)}\right]_{D_{1}^{(6)} Q_{9, i}^{(6)}}=\left[\gamma_{2}^{(0)}\right]_{D_{2}^{(6)} Q_{12, i}^{(6)}} \\
& =\left[\gamma_{2}^{(0)}\right]_{D_{1}^{(6)} Q_{15}^{(6)}}=\left[\gamma_{2}^{(0)}\right]_{D_{2}^{(6)} Q_{17}^{(6)}}=\frac{8}{3}, \\
{\left[\gamma_{2}^{(0)}\right]_{D_{3}^{(6)} Q_{1, i}^{(6)}} } & =\left[\gamma_{2}^{(0)}\right]_{D_{3}^{(6)} Q_{9, i}^{(6)}}=\left[\gamma_{2}^{(0)}\right]_{D_{3}^{(6)} Q_{15}^{(6)}}=\left(\frac{8}{3}+\frac{16}{9} d_{\chi}\right) \mathcal{J}_{\chi}-\frac{8}{3}, \\
{\left[\gamma_{2}^{(0)}\right]_{D_{4}^{(6)} Q_{5, i}^{(6)}} } & =\left[\gamma_{2}^{(0)}\right]_{D_{4}^{(6)} Q_{12, i}^{(6)}}=\left[\gamma_{2}^{(0)}\right]_{D_{4}^{(6)} Q_{17}^{(6)}}=\left(\frac{8}{3}+\frac{8}{9} d_{\chi}\right) \mathcal{J}_{\chi}-\frac{8}{3} .
\end{aligned}
$$

All non-displayed entries vanish.

\section{E Unphysical operators}

We extract the anomalous dimensions by renormalizing off-shell Greens functions in $d=$ $4-2 \epsilon$ dimensions. In the intermediate stages of the computation it is thus necessary to introduce unphysical operators. 


\section{E.1 Evanescent operators}

The one-loop mixing among the "physical" operators is not affected by the definition of evanescent operators, i.e., operators that are required to project one-loop Green's functions in $d=4-2 \epsilon$ dimensions but vanish in $d=4$. Nevertheless, for completeness and future reference we list below the ones we used for the one-loop computations. The evanescent operators with quark fields are chosen as

$$
\begin{aligned}
& E_{1, i}^{(6)}=\left(\bar{\chi} \gamma_{\mu} \gamma_{\nu} \gamma_{\rho} \tilde{\tau}^{a} \chi\right)\left(\bar{Q}_{L}^{i} \gamma^{\mu} \gamma^{\nu} \gamma^{\rho} \tau^{a} Q_{L}^{i}\right)-10 Q_{1, i}^{(6)}+6 Q_{5, i}^{(6)} \\
& E_{2, i}^{(6)}=\left(\bar{\chi} \gamma_{\mu} \gamma_{\nu} \gamma_{\rho} \gamma_{5} \tilde{\tau}^{a} \chi\right)\left(\bar{Q}_{L}^{i} \gamma^{\mu} \gamma^{\nu} \gamma^{\rho} \tau^{a} Q_{L}^{i}\right)+6 Q_{1, i}^{(6)}-10 Q_{5, i}^{(6)} \\
& E_{3, i}^{(6)}=\left(\bar{\chi} \gamma_{\mu} \gamma_{\nu} \gamma_{\rho} \chi\right)\left(\bar{Q}_{L}^{i} \gamma^{\mu} \gamma^{\nu} \gamma^{\rho} Q_{L}^{i}\right)-10 Q_{2, i}^{(6)}+6 Q_{6, i}^{(6)} \\
& E_{4, i}^{(6)}=\left(\bar{\chi} \gamma_{\mu} \gamma_{\nu} \gamma_{\rho} \gamma_{5} \chi\right)\left(\bar{Q}_{L}^{i} \gamma^{\mu} \gamma^{\nu} \gamma^{\rho} Q_{L}^{i}\right)+6 Q_{2, i}^{(6)}-10 Q_{6, i}^{(6)} \\
& E_{5, i}^{(6)}=\left(\bar{\chi} \gamma_{\mu} \gamma_{\nu} \gamma_{\rho} \chi\right)\left(\bar{u}_{R}^{i} \gamma^{\mu} \gamma^{\nu} \gamma^{\rho} u_{R}^{i}\right)-10 Q_{3, i}^{(6)}-6 Q_{7, i}^{(6)} \\
& E_{6, i}^{(6)}=\left(\bar{\chi} \gamma_{\mu} \gamma_{\nu} \gamma_{\rho} \gamma_{5} \chi\right)\left(\bar{u}_{R}^{i} \gamma^{\mu} \gamma^{\nu} \gamma^{\rho} u_{R}^{i}\right)-6 Q_{3, i}^{(6)}-10 Q_{7, i}^{(6)} \\
& E_{7, i}^{(6)}=\left(\bar{\chi} \gamma_{\mu} \gamma_{\nu} \gamma_{\rho} \chi\right)\left(\bar{d}_{R}^{i} \gamma^{\mu} \gamma^{\nu} \gamma^{\rho} d_{R}^{i}\right)-10 Q_{4, i}^{(6)}-6 Q_{8, i}^{(6)} \\
& E_{8, i}^{(6)}=\left(\bar{\chi} \gamma_{\mu} \gamma_{\nu} \gamma_{\rho} \gamma_{5} \chi\right)\left(\bar{d}_{R}^{i} \gamma^{\mu} \gamma^{\nu} \gamma^{\rho} d_{R}^{i}\right)-6 Q_{4, i}^{(6)}-10 Q_{8, i}^{(6)}
\end{aligned}
$$

while the evanescent operators involving lepton fields are

$$
\begin{aligned}
& E_{9, i}^{(6)}=\left(\bar{\chi} \gamma_{\mu} \gamma_{\nu} \gamma_{\rho} \tilde{\tau}^{a} \chi\right)\left(\bar{L}_{L}^{i} \gamma^{\mu} \gamma^{\nu} \gamma^{\rho} \tau^{a} L_{L}^{i}\right)-10 Q_{9, i}^{(6)}+6 Q_{12, i}^{(6)}, \\
& E_{10, i}^{(6)}=\left(\bar{\chi} \gamma_{\mu} \gamma_{\nu} \gamma_{\rho} \gamma_{5} \tilde{\tau}^{a} \chi\right)\left(\bar{L}_{L}^{i} \gamma^{\mu} \gamma^{\nu} \gamma^{\rho} \tau^{a} L_{L}^{i}\right)+6 Q_{9, i}^{(6)}-10 Q_{12, i}^{(6)}, \\
& E_{11, i}^{(6)}=\left(\bar{\chi} \gamma_{\mu} \gamma_{\nu} \gamma_{\rho} \chi\right)\left(\bar{L}_{L}^{i} \gamma^{\mu} \gamma^{\nu} \gamma^{\rho} L_{L}^{i}\right)-10 Q_{10, i}^{(6)}+6 Q_{13, i}^{(6)}, \\
& E_{12, i}^{(6)}=\left(\bar{\chi} \gamma_{\mu} \gamma_{\nu} \gamma_{\rho} \gamma_{5} \chi\right)\left(\bar{L}_{L}^{i} \gamma^{\mu} \gamma^{\nu} \gamma^{\rho} L_{L}^{i}\right)+6 Q_{10, i}^{(6)}-10 Q_{13, i}^{(6)}, \\
& E_{13, i}^{(6)}=\left(\bar{\chi} \gamma_{\mu} \gamma_{\nu} \gamma_{\rho} \chi\right)\left(\bar{\ell}_{R}^{i} \gamma^{\mu} \gamma^{\nu} \gamma^{\rho} \ell_{R}^{i}\right)-10 Q_{11, i}^{(6)}-6 Q_{14, i}^{(6)}, \\
& E_{14, i}^{(6)}=\left(\bar{\chi} \gamma_{\mu} \gamma_{\nu} \gamma_{\rho} \gamma_{5} \chi\right)\left(\bar{\ell}_{R}^{i} \gamma^{\mu} \gamma^{\nu} \gamma^{\rho} \ell_{R}^{i}\right)-6 Q_{11, i}^{(6)}-10 Q_{14, i}^{(6)} .
\end{aligned}
$$

\section{E.2 EOM - Vanishing operators}

The equations of motion (EOM) for the $W$ and $B$ gauge-boson field are, in our conventions,

$$
D^{\nu} W_{\nu \mu}^{a} \equiv\left(\partial^{\nu} \delta^{a b}-g_{2} \epsilon^{a b c} W^{\nu, c}\right) W_{\nu \mu}^{b}=-g_{2} \sum_{\psi} \bar{\psi} \tilde{\tau}^{a} \gamma_{\mu} \psi-i g_{2} H^{\dagger} \stackrel{\leftrightarrow}{D_{\mu}^{a}} H
$$

and

$$
D^{\nu} B_{\nu \mu} \equiv \partial^{\nu} B_{\nu \mu}=g_{1} \sum_{\psi} \frac{Y}{2} \bar{\psi} \gamma_{\mu} \psi+i \frac{g_{1}}{2} H^{\dagger} \stackrel{\leftrightarrow}{D}_{\mu} H
$$

up to gauge-fixing and ghost terms (see ref. [107] for a more detailed discussion of the EOM in effective theories.). The sum is over all active fermion fields. 
The following operators vanish via the EOM of the gauge fields; they contribute to the same amplitudes as the physical four-fermion operators. Therefore, the mixing of physical operators into the EOM-vanishing operators (computed from penguin diagrams) affects the anomalous dimensions of four-fermion operators. There are four operators involving DM currents,

$$
\begin{aligned}
P_{1}^{(6)}= & \frac{1}{g_{2}}\left(\bar{\chi} \gamma_{\mu} \tilde{\tau}^{a} \chi\right) D_{\nu} W^{a, \nu \mu}+\sum_{i}\left(Q_{1, i}^{(6)}+Q_{9, i}^{(6)}\right)+Q_{15}^{(6)}+D_{3}^{(6)}, \\
P_{2}^{(6)}= & \frac{1}{g_{1}}\left(\bar{\chi} \gamma_{\mu} \chi\right) D_{\nu} B^{\nu \mu} \\
& -\sum_{i}\left(\frac{1}{6} Q_{2, i}^{(6)}+\frac{2}{3} Q_{3, i}^{(6)}-\frac{1}{3} Q_{4, i}^{(6)}-\frac{1}{2} Q_{10, i}^{(6)}-Q_{11, i}^{(6)}\right)-\frac{1}{2} Q_{16}^{(6)}-\frac{Y_{\chi}}{2} D_{1}^{(6)}, \\
P_{3}^{(6)}= & \frac{1}{g_{2}}\left(\bar{\chi} \gamma_{\mu} \gamma_{5} \tilde{\tau}^{a} \chi\right) D_{\nu} W^{a, \nu \mu}+\sum_{i}\left(Q_{5, i}^{(6)}+Q_{12, i}^{(6)}\right)+Q_{17}^{(6)}+D_{4}^{(6)} \\
P_{4}^{(6)}= & \frac{1}{g_{1}}\left(\bar{\chi} \gamma_{\mu} \gamma_{5} \chi\right) D_{\nu} B^{\nu \mu} \\
& -\sum_{i}\left(\frac{1}{6} Q_{6, i}^{(6)}+\frac{2}{3} Q_{7, i}^{(6)}-\frac{1}{3} Q_{8, i}^{(6)}-\frac{1}{2} Q_{13, i}^{(6)}-Q_{14, i}^{(6)}\right)-\frac{1}{2} Q_{18}^{(6)}-\frac{Y_{\chi}}{2} D_{2}^{(6)},
\end{aligned}
$$

four operators involving quark currents,

$$
\begin{aligned}
P_{5, i}^{(6)}= & \frac{1}{g_{2}}\left(\bar{Q}_{L}^{i} \gamma^{\mu} \tilde{\tau}^{a} Q_{L}^{i}\right) D_{\nu} W^{a, \nu \mu}+Q_{1, i}^{(6)}+\sum_{j}\left(S_{1, i j}^{(6)}+S_{11, i j}^{(6)}\right)+S_{18, i}^{(6)}, \\
P_{6, i}^{(6)}= & \frac{1}{g_{1}}\left(\bar{Q}_{L}^{i} \gamma^{\mu} Q_{L}^{i}\right) D_{\nu} B^{\nu \mu}-\frac{Y_{\chi}}{2} Q_{2, i}^{(6)} \\
& -\sum_{j}\left(\frac{1}{6} S_{2, i j}^{(6)}+\frac{2}{3} S_{3, i j}^{(6)}-\frac{1}{3} S_{4, i j}^{(6)}-\frac{1}{2} S_{12, i j}^{(6)}-S_{13, i j}^{(6)}\right)-\frac{1}{2} S_{19, i}^{(6)}, \\
P_{7, i}^{(6)}= & \frac{1}{g_{1}}\left(\bar{u}_{R}^{i} \gamma^{\mu} u_{R}^{i}\right) D_{\nu} B^{\nu \mu}-\frac{Y_{\chi}}{2} Q_{3, i}^{(6)} \\
& -\sum_{j}\left(\frac{1}{6} S_{3, j i}^{(6)}+\frac{2}{3} S_{5, i j}^{(6)}-\frac{1}{3} S_{6, i j}^{(6)}-\frac{1}{2} S_{14, i j}^{(6)}-S_{16, i j}^{(6)}\right)-\frac{1}{2} S_{20, i}^{(6)}, \\
P_{8, i}^{(6)}= & \frac{1}{g_{1}}\left(\bar{d}_{R}^{i} \gamma^{\mu} d_{R}^{i}\right) D_{\nu} B^{\nu \mu}-\frac{Y_{\chi}}{2} Q_{4, i}^{(6)} \\
& -\sum_{j}\left(\frac{1}{6} S_{4, j i}^{(6)}+\frac{2}{3} S_{6, j i}^{(6)}-\frac{1}{3} S_{7, i j}^{(6)}-\frac{1}{2} S_{15, i j}^{(6)}-S_{17, i j}^{(6)}\right)-\frac{1}{2} S_{21, i}^{(6)},
\end{aligned}
$$

two operators involving Higgs currents,

$$
\begin{aligned}
P_{9}^{(6)}= & \frac{1}{g_{2}}\left[H^{\dagger} i \stackrel{\leftrightarrow}{D_{\mu}^{a}} H\right] D_{\nu} W^{a, \nu \mu}+Q_{15}^{(6)}+\sum_{i}\left(S_{18, i}^{(6)}+S_{22, i}^{(6)}\right)+\frac{1}{4} S_{25}^{(6)} \\
P_{10}^{(6)}= & \frac{1}{g_{1}}\left(H^{\dagger} i \stackrel{\leftrightarrow}{D} \mu H\right) D_{\nu} B^{\nu \mu}-\frac{Y_{\chi}}{2} Q_{16}^{(6)} \\
& -\sum_{i}\left(\frac{1}{6} S_{19, i}^{(6)}+\frac{2}{3} S_{20, i}^{(6)}-\frac{1}{3} S_{21, i}^{(6)}-\frac{1}{2} S_{23, i}^{(6)}-S_{24, i}^{(6)}\right)-\frac{1}{2} S_{25}^{(6)},
\end{aligned}
$$


and three operators involving lepton currents,

$$
\begin{aligned}
P_{11, i}^{(6)}= & \frac{1}{g_{2}}\left(\bar{L}_{L}^{i} \gamma^{\mu} \tilde{\tau}^{a} L_{L}^{i}\right) D_{\nu} W^{a, \nu \mu}+Q_{9, i}^{(6)}+\sum_{j}\left(\frac{1}{4} S_{8, i j}^{(6)}+S_{11, j i}^{(6)}\right)+S_{22, i}^{(6)}, \\
P_{12, i}^{(6)}= & \frac{1}{g_{1}}\left(\bar{L}_{L}^{i} \gamma^{\mu} L_{L}^{i}\right) D_{\nu} B^{\nu \mu}-\frac{Y_{\chi}}{2} Q_{10, i}^{(6)} \\
& -\sum_{j}\left(\frac{1}{6} S_{12, j i}^{(6)}+\frac{2}{3} S_{14, j i}^{(6)}-\frac{1}{3} S_{15, j i}^{(6)}-\frac{1}{2} S_{8, i j}^{(6)}-S_{9, i j}^{(6)}\right)-\frac{1}{2} S_{23, i}^{(6)}, \\
P_{13, i}^{(6)}= & \frac{1}{g_{1}}\left(\bar{\ell}_{R}^{i} \gamma^{\mu} \ell_{R}^{i}\right) D_{\nu} B^{\nu \mu}-\frac{Y_{\chi}}{2} Q_{11, i}^{(6)} \\
& -\sum_{j}\left(\frac{1}{6} S_{13, j i}^{(6)}+\frac{2}{3} S_{16, j i}^{(6)}-\frac{1}{3} S_{17, j i}^{(6)}-\frac{1}{2} S_{9, j i}^{(6)}-S_{10, i j}^{(6)}\right)-\frac{1}{2} S_{24, i}^{(6)} .
\end{aligned}
$$

Several additional operators, vanishing due to the EOM for the DM fields, are needed to project all one-loop Greens functions with insertions of the operators in eqs. (2.5), (2.7) and eqs. (2.16), (2.17), respectively: two dimension-five operators,

$$
P_{1}^{(5)}=\bar{\chi} \not D \not D \chi, \quad P_{2}^{(5)}=\bar{\chi} \not D \not D i \gamma_{5} \chi,
$$

and eight dimension-six operators,

$$
\begin{aligned}
P_{14}^{(6)} & =\left(\bar{\chi} \tilde{\tau}^{a} i \not D \chi\right)\left(H^{\dagger} \tau^{a} H\right), & P_{15}^{(6)} & =\left(\bar{\chi} i \overleftarrow{D D}^{\dagger} \tilde{\tau}^{a} \chi\right)\left(H^{\dagger} \tau^{a} H\right), \\
P_{16}^{(6)} & =(\bar{\chi} i \not D \chi)\left(H^{\dagger} H\right), & P_{17}^{(6)} & =\left(\bar{\chi} i \overleftarrow{D D}^{\dagger} \chi\right)\left(H^{\dagger} H\right), \\
P_{18}^{(6)} & =\left(\bar{\chi} \tilde{\tau}^{a} i \not D \gamma_{5} \chi\right)\left(H^{\dagger} \tau^{a} H\right), & P_{19}^{(6)} & =\left(\bar{\chi} i \overleftarrow{D D}^{\dagger} \gamma_{5} \tilde{\tau}^{a} \chi\right)\left(H^{\dagger} \tau^{a} H\right) \\
P_{20}^{(6)} & =\left(\bar{\chi} i \not D \gamma_{5} \chi\right)\left(H^{\dagger} H\right), & P_{21}^{(6)} & =\left(\bar{\chi} i \overleftarrow{D D}^{\dagger} \gamma_{5} \chi\right)\left(H^{\dagger} H\right) .
\end{aligned}
$$

Open Access. This article is distributed under the terms of the Creative Commons Attribution License (CC-BY 4.0), which permits any use, distribution and reproduction in any medium, provided the original author(s) and source are credited.

\section{References}

[1] J. Bagnasco, M. Dine and S.D. Thomas, Detecting technibaryon dark matter, Phys. Lett. B 320 (1994) 99 [hep-ph/9310290] [INSPIRE].

[2] M. Pospelov and T. ter Veldhuis, Direct and indirect limits on the electromagnetic form-factors of WIMPs, Phys. Lett. B 480 (2000) 181 [hep-ph/0003010] [INSPIRE].

[3] A. Kurylov and M. Kamionkowski, Generalized analysis of weakly interacting massive particle searches, Phys. Rev. D 69 (2004) 063503 [hep-ph/0307185] [INSPIRE].

[4] J. Kopp, T. Schwetz and J. Zupan, Global interpretation of direct Dark Matter searches after CDMS-II results, JCAP 02 (2010) 014 [arXiv:0912.4264] [INSPIRE].

[5] J. Fan, M. Reece and L.-T. Wang, Non-relativistic effective theory of dark matter direct detection, JCAP 11 (2010) 042 [arXiv: 1008.1591] [INSPIRE]. 
[6] V. Cirigliano, M.L. Graesser and G. Ovanesyan, WIMP-nucleus scattering in chiral effective theory, JHEP 10 (2012) 025 [arXiv: 1205.2695] [INSPIRE].

[7] R.J. Hill and M.P. Solon, Universal behavior in the scattering of heavy, weakly interacting dark matter on nuclear targets, Phys. Lett. B 707 (2012) 539 [arXiv:1111.0016] [InSPIRE].

[8] R.J. Hill and M.P. Solon, WIMP-nucleon scattering with heavy WIMP effective theory, Phys. Rev. Lett. 112 (2014) 211602 [arXiv:1309.4092] [INSPIRE].

[9] A.L. Fitzpatrick et al., The effective field theory of dark matter direct detection, JCAP 02 (2013) 004 [arXiv: 1203.3542] [INSPIRE].

[10] A.L. Fitzpatrick et al., Model independent direct detection analyses, arXiv:1211.2818 [inSPIRE].

[11] J. Menendez, D. Gazit and A. Schwenk, Spin-dependent WIMP scattering off nuclei, Phys. Rev. D 86 (2012) 103511 [arXiv:1208.1094] [INSPIRE].

[12] N. Anand, A.L. Fitzpatrick and W.C. Haxton, Weakly interacting massive particle-nucleus elastic scattering response, Phys. Rev. C 89 (2014) 065501 [arXiv:1308.6288] [INSPIRE].

[13] P. Klos, J. Menéndez, D. Gazit and A. Schwenk, Large-scale nuclear structure calculations for spin-dependent WIMP scattering with chiral effective field theory currents, Phys. Rev. D 88 (2013) 083516 [Erratum ibid. D 89 (2014) 029901] [arXiv: 1304.7684] [INSPIRE].

[14] M. Cirelli, E. Del Nobile and P. Panci, Tools for model-independent bounds in direct dark matter searches, JCAP 10 (2013) 019 [arXiv:1307.5955] [INSPIRE].

[15] G. Barello, S. Chang and C.A. Newby, A model independent approach to inelastic dark matter scattering, Phys. Rev. D 90 (2014) 094027 [arXiv: 1409.0536] [INSPIRE].

[16] R.J. Hill and M.P. Solon, Standard Model anatomy of WIMP dark matter direct detection II: QCD analysis and hadronic matrix elements, Phys. Rev. D 91 (2015) 043505 [arXiv: 1409.8290] [INSPIRE].

[17] R. Catena and P. Gondolo, Global fits of the dark matter-nucleon effective interactions, JCAP 09 (2014) 045 [arXiv: 1405.2637] [INSPIRE].

[18] M. Hoferichter, P. Klos and A. Schwenk, Chiral power counting of one- and two-body currents in direct detection of dark matter, Phys. Lett. B 746 (2015) 410 [arXiv: 1503.04811] [INSPIRE].

[19] M. Hoferichter, P. Klos, J. Menéndez and A. Schwenk, Analysis strategies for general spin-independent WIMP-nucleus scattering, Phys. Rev. D 94 (2016) 063505 [arXiv: 1605. 08043] [INSPIRE].

[20] F. Bishara, J. Brod, B. Grinstein and J. Zupan, Chiral effective theory of dark matter direct detection, JCAP 02 (2017) 009 [arXiv: 1611.00368] [INSPIRE].

[21] F. Bishara, J. Brod, B. Grinstein and J. Zupan, From quarks to nucleons in dark matter direct detection, JHEP 11 (2017) 059 [arXiv: 1707.06998] [INSPIRE].

[22] F. D'Eramo, B.J. Kavanagh and P. Panci, You can hide but you have to run: direct detection with vector mediators, JHEP 08 (2016) 111 [arXiv: 1605.04917] [INSPIRE].

[23] F. Bishara, J. Brod, B. Grinstein and J. Zupan, DirectDM: a tool for dark matter direct detection, arXiv:1708.02678 [INSPIRE].

[24] F. D'Eramo, B.J. Kavanagh and P. Panci, Probing leptophilic dark sectors with hadronic processes, Phys. Lett. B 771 (2017) 339 [arXiv:1702.00016] [INSPIRE]. 
[25] J. Brod, B. Grinstein, E. Stamou and J. Zupan, Weak mixing below the weak scale in dark-matter direct detection, JHEP 02 (2018) 174 [arXiv: 1801.04240] [INSPIRE].

[26] J. Brod, A. Gootjes-Dreesbach, M. Tammaro and J. Zupan, Effective field theory for dark matter direct detection up to dimension seven, JHEP 10 (2018) 065 [arXiv:1710.10218] [INSPIRE].

[27] C.-Y. Chen, R.J. Hill, M.P. Solon and A.M. Wijangco, Power corrections to the universal heavy WIMP-nucleon cross section, Phys. Lett. B 781 (2018) 473 [arXiv:1801.08551] [INSPIRE].

[28] J. Kumar and D. Marfatia, Matrix element analyses of dark matter scattering and annihilation, Phys. Rev. D 88 (2013) 014035 [arXiv:1305.1611] [INSPIRE].

[29] Q.-H. Cao, C.-R. Chen, C.S. Li and H. Zhang, Effective dark matter model: relic density, CDMS II, Fermi LAT and LHC, JHEP 08 (2011) 018 [arXiv:0912.4511] [INSPIRE].

[30] J. Goodman et al., Gamma ray line constraints on effective theories of dark matter, Nucl. Phys. B 844 (2011) 55 [arXiv: 1009.0008] [INSPIRE].

[31] P. Ciafaloni et al., On the importance of electroweak corrections for Majorana dark matter indirect detection, JCAP 06 (2011) 018 [arXiv:1104.2996] [INSPIRE].

[32] K. Cheung, P.-Y. Tseng and T.-C. Yuan, Gamma-ray constraints on effective interactions of the dark matter, JCAP 06 (2011) 023 [arXiv:1104.5329] [INSPIRE].

[33] K. Cheung, P.-Y. Tseng, Y.-L.S. Tsai and T.-C. Yuan, Global constraints on effective dark matter interactions: relic density, direct detection, indirect detection and collider, JCAP 05 (2012) 001 [arXiv: 1201.3402] [INSPIRE].

[34] L. Vietze et al., Nuclear structure aspects of spin-independent WIMP scattering off xenon, Phys. Rev. D 91 (2015) 043520 [arXiv:1412.6091] [InSPIRE].

[35] U. Haisch and E. Re, Simplified dark matter top-quark interactions at the LHC, JHEP 06 (2015) 078 [arXiv: 1503.00691] [inSPIRE].

[36] R.C. Cotta, J.L. Hewett, M.P. Le and T.G. Rizzo, Bounds on dark matter interactions with electroweak gauge bosons, Phys. Rev. D 88 (2013) 116009 [arXiv:1210.0525] [INSPIRE].

[37] G. Busoni, A. De Simone, E. Morgante and A. Riotto, On the validity of the effective field theory for dark matter searches at the LHC, Phys. Lett. B 728 (2014) 412 [arXiv: 1307.2253] [INSPIRE].

[38] P.J. Fox, R. Harnik, J. Kopp and Y. Tsai, LEP shines light on dark matter, Phys. Rev. D 84 (2011) 014028 [arXiv: 1103.0240] [INSPIRE].

[39] A. Rajaraman, W. Shepherd, T.M.P. Tait and A.M. Wijangco, LHC bounds on interactions of dark matter, Phys. Rev. D 84 (2011) 095013 [arXiv:1108.1196] [INSPIRE].

[40] P.J. Fox, R. Harnik, J. Kopp and Y. Tsai, Missing energy signatures of dark matter at the LHC, Phys. Rev. D 85 (2012) 056011 [arXiv:1109.4398] [InSPIRE].

[41] D. Racco, A. Wulzer and F. Zwirner, Robust collider limits on heavy-mediator dark matter, JHEP 05 (2015) 009 [arXiv: 1502.04701] [InSPIRE].

[42] T. Jacques and K. Nordström, Mapping monojet constraints onto simplified dark matter models, JHEP 06 (2015) 142 [arXiv:1502.05721] [INSPIRE].

[43] M. Bauer et al., Validity of dark matter effective theory, Phys. Rev. D 95 (2017) 075036 [arXiv: 1611.09908] [INSPIRE]. 
[44] A. Albert et al., Towards the next generation of simplified Dark Matter models, Phys. Dark Univ. 16 (2017) 49 [arXiv:1607.06680] [INSPIRE].

[45] J. Abdallah et al., Simplified models for dark matter searches at the LHC, Phys. Dark Univ. 9-10 (2015) 8 [arXiv:1506.03116] [INSPIRE].

[46] S. Bruggisser, F. Riva and A. Urbano, The last gasp of dark matter effective theory, JHEP 11 (2016) 069 [arXiv:1607.02475] [InSPIRE].

[47] A. De Simone and T. Jacques, Simplified models vs. effective field theory approaches in dark matter searches, Eur. Phys. J. C 76 (2016) 367 [arXiv: 1603.08002] [InSPIRE].

[48] F. Kahlhoefer, K. Schmidt-Hoberg, T. Schwetz and S. Vogl, Implications of unitarity and gauge invariance for simplified dark matter models, JHEP 02 (2016) 016

[arXiv: 1510.02110$]$ [INSPIRE].

[49] G. Busoni et al., Recommendations on presenting LHC searches for missing transverse energy signals using simplified s-channel models of dark matter, Phys. Dark Univ. 27 (2020) 100365 [arXiv: 1603.04156] [INSPIRE].

[50] D. Goncalves, P.A.N. Machado and J.M. No, Simplified models for dark matter face their consistent completions, Phys. Rev. D 95 (2017) 055027 [arXiv: 1611.04593] [INSPIRE].

[51] N.F. Bell, G. Busoni and I.W. Sanderson, Self-consistent dark matter simplified models with an s-channel scalar mediator, JCAP 03 (2017) 015 [arXiv: 1612.03475] [INSPIRE].

[52] M. Duerr et al., How to save the WIMP: global analysis of a dark matter model with two s-channel mediators, JHEP 09 (2016) 042 [arXiv:1606.07609] [INSPIRE].

[53] C. Englert, M. McCullough and M. Spannowsky, S-channel dark matter simplified models and unitarity, Phys. Dark Univ. 14 (2016) 48 [arXiv:1604.07975] [InSPIRE].

[54] A.J. Brennan, M.F. McDonald, J. Gramling and T.D. Jacques, Collide and conquer: constraints on simplified dark matter models using Mono-X collider searches, JHEP 05 (2016) 112 [arXiv: 1603.01366] [INSPIRE].

[55] T. Alanne and F. Goertz, Extended dark matter EFT, arXiv:1712.07626 [INSPIRE].

[56] G. Bertone et al., Identifying WIMP dark matter from particle and astroparticle data, JCAP 03 (2018) 026 [arXiv: 1712.04793] [INSPIRE].

[57] E. Bernreuther, J. Horak, T. Plehn and A. Butter, Actual physics behind Mono-X, SciPost Phys. 5 (2018) 034 [arXiv:1805.11637] [INSPIRE].

[58] D. Gazda, R. Catena and C. Forssén, Ab initio nuclear response functions for dark matter searches, Phys. Rev. D 95 (2017) 103011 [arXiv:1612.09165] [INSPIRE].

[59] C. Körber, A. Nogga and J. de Vries, First-principle calculations of Dark Matter scattering off light nuclei, Phys. Rev. C 96 (2017) 035805 [arXiv: 1704.01150] [InSPIRE].

[60] A. Crivellin, F. D'Eramo and M. Procura, New constraints on dark matter effective theories from standard model loops, Phys. Rev. Lett. 112 (2014) 191304 [arXiv:1402.1173] [INSPIRE].

[61] F. D'Eramo and M. Procura, Connecting dark matter UV complete models to direct detection rates via effective field theory, JHEP 04 (2015) 054 [arXiv: 1411.3342] [INSPIRE].

[62] A. Crivellin, U. Haisch and A. Hibbs, LHC constraints on gauge boson couplings to dark matter, Phys. Rev. D 91 (2015) 074028 [arXiv: 1501.00907] [InSPIRE]. 
[63] U. Haisch, F. Kahlhoefer and E. Re, QCD effects in mono-jet searches for dark matter, JHEP 12 (2013) 007 [arXiv:1310.4491] [InSPIRE].

[64] U. Haisch and F. Kahlhoefer, On the importance of loop-induced spin-independent interactions for dark matter direct detection, JCAP 04 (2013) 050 [arXiv: 1302.4454] [INSPIRE].

[65] U. Haisch, F. Kahlhoefer and J. Unwin, The impact of heavy-quark loops on LHC dark matter searches, JHEP 07 (2013) 125 [arXiv: 1208.4605] [INSPIRE].

[66] M.T. Frandsen et al., Loop-induced dark matter direct detection signals from gamma-ray lines, JCAP 10 (2012) 033 [arXiv:1207.3971] [INSPIRE].

[67] M. Freytsis and Z. Ligeti, On dark matter models with uniquely spin-dependent detection possibilities, Phys. Rev. D 83 (2011) 115009 [arXiv: 1012.5317] [INSPIRE].

[68] J. Hisano, K. Ishiwata, N. Nagata and T. Takesako, Direct detection of electroweak-interacting dark matter, JHEP 07 (2011) 005 [arXiv: 1104.0228] [INSPIRE].

[69] J. Hisano, K. Ishiwata and N. Nagata, Gluon contribution to the dark matter direct detection, Phys. Rev. D 82 (2010) 115007 [arXiv: 1007.2601] [INSPIRE].

[70] M. Cirelli, N. Fornengo and A. Strumia, Minimal dark matter, Nucl. Phys. B 753 (2006) 178 [hep-ph/0512090] [INSPIRE].

[71] Y. Bai and J. Osborne, Chromo-Rayleigh interactions of dark matter, JHEP 11 (2015) 036 [arXiv: 1506.07110] [INSPIRE].

[72] N. Weiner and I. Yavin, UV completions of magnetic inelastic and Rayleigh dark matter for the Fermi Line(s), Phys. Rev. D 87 (2013) 023523 [arXiv: 1209.1093] [InSPIRE].

[73] G. Buchalla, A.J. Buras and M.E. Lautenbacher, Weak decays beyond leading logarithms, Rev. Mod. Phys. 68 (1996) 1125 [hep-ph/9512380] [INSPIRE].

[74] P. Nogueira, Automatic Feynman graph generation, J. Comput. Phys. 105 (1993) 279.

[75] J.A.M. Vermaseren, New features of FORM, math-ph/0010025 [INSPIRE].

[76] A. Alloul et al., FeynRules 2.0 - A complete toolbox for tree-level phenomenology, Comput. Phys. Commun. 185 (2014) 2250 [arXiv:1310.1921] [InSPIRE].

[77] T. Hahn, Generating Feynman diagrams and amplitudes with FeynArts 3, Comput. Phys. Commun. 140 (2001) 418 [hep-ph/0012260] [INSPIRE].

[78] T. Hahn and M. Pérez-Victoria, Automatized one loop calculations in four-dimensions and D-dimensions, Comput. Phys. Commun. 118 (1999) 153 [hep-ph/9807565] [INSPIRE].

[79] M.J. Dugan and B. Grinstein, On the vanishing of evanescent operators, Phys. Lett. B 256 (1991) 239 [INSPIRE].

[80] J.C. Collins, A.V. Manohar and M.B. Wise, Renormalization of the vector current in QED, Phys. Rev. D 73 (2006) 105019 [hep-th/0512187] [INSPIRE].

[81] R.J. Hill and M.P. Solon, Standard Model anatomy of WIMP dark matter direct detection I: weak-scale matching, Phys. Rev. D 91 (2015) 043504 [arXiv:1401.3339] [INSPIRE].

[82] M.A. Fedderke, J.-Y. Chen, E.W. Kolb and L.-T. Wang, The Fermionic dark matter Higgs portal: an effective field theory approach, JHEP 08 (2014) 122 [arXiv:1404.2283] [INSPIRE].

[83] B. Grinstein, The static quark effective theory, Nucl. Phys. B 339 (1990) 253 [INSPIRE]. 
[84] E. Eichten and B.R. Hill, An effective field theory for the calculation of matrix elements involving heavy quarks, Phys. Lett. B 234 (1990) 511 [INSPIRE].

[85] H. Georgi, An effective field theory for heavy quarks at low-energies, Phys. Lett. B 240 (1990) 447 [INSPIRE].

[86] A. Berlin, D.S. Robertson, M.P. Solon and K.M. Zurek, Bino variations: effective field theory methods for dark matter direct detection, Phys. Rev. D 93 (2016) 095008 [arXiv:1511.05964] [INSPIRE].

[87] M.E. Luke and A.V. Manohar, Reparametrization invariance constraints on heavy particle effective field theories, Phys. Lett. B 286 (1992) 348 [hep-ph/9205228] [INSPIRE].

[88] J. Hisano, R. Nagai and N. Nagata, Effective theories for dark matter nucleon scattering, JHEP 05 (2015) 037 [arXiv: 1502.02244] [INSPIRE].

[89] J. Kopp, V. Niro, T. Schwetz and J. Zupan, DAMA/LIBRA and leptonically interacting Dark Matter, Phys. Rev. D 80 (2009) 083502 [arXiv:0907.3159] [InSPIRE].

[90] K.G. Chetyrkin, B.A. Kniehl and M. Steinhauser, Decoupling relations to $O\left(\alpha_{S}^{3}\right)$ and their connection to low-energy theorems, Nucl. Phys. B 510 (1998) 61 [hep-ph/9708255] [INSPIRE].

[91] L. Baudis et al., Signatures of dark matter scattering inelastically off nuclei, Phys. Rev. D 88 (2013) 115014 [arXiv: 1309.0825] [INSPIRE].

[92] T. Banks, J.-F. Fortin and S. Thomas, Direct detection of dark matter electromagnetic dipole moments, arXiv:1007.5515 [INSPIRE].

[93] S. Kang, S. Scopel, G. Tomar and J.-H. Yoon, Present and projected sensitivities of Dark Matter direct detection experiments to effective WIMP-nucleus couplings, Astropart. Phys. 109 (2019) 50 [arXiv:1805.06113] [INSPIRE].

[94] QCDSF collaboration, Strangeness contribution to the proton spin from lattice QCD, Phys. Rev. Lett. 108 (2012) 222001 [arXiv:1112.3354] [INSPIRE].

[95] M. Engelhardt, Strange quark contributions to nucleon mass and spin from lattice QCD, Phys. Rev. D 86 (2012) 114510 [arXiv:1210.0025] [INSPIRE].

[96] T. Bhattacharya, R. Gupta and B. Yoon, Disconnected quark loop contributions to nucleon structure, PoS (LATTICE2014) 141 [arXiv:1503.05975] [INSPIRE].

[97] C. Alexandrou et al., Nucleon axial form factors using $N_{f}=2$ twisted mass fermions with a physical value of the pion mass, Phys. Rev. D 96 (2017) 054507 [arXiv:1705. 03399] [INSPIRE].

[98] M.V. Polyakov, A. Schafer and O.V. Teryaev, The intrinsic charm contribution to the proton spin, Phys. Rev. D 60 (1999) 051502 [hep-ph/9812393] [INSPIRE].

[99] L.F. Abbott, The background field method beyond one loop, Nucl. Phys. B 185 (1981) 189 [INSPIRE].

[100] L.F. Abbott, Introduction to the background field method, Acta Phys. Polon. B 13 (1982) 33 [INSPIRE].

[101] A. Denner, G. Weiglein and S. Dittmaier, Application of the background field method to the electroweak standard model, Nucl. Phys. B 440 (1995) 95 [hep-ph/9410338] [InSPIRE].

[102] Particle Data Group collaboration, Review of particle physics, Phys. Rev. D 98 (2018) 030001 [INSPIRE]. 
[103] B. Grzadkowski, M. Iskrzynski, M. Misiak and J. Rosiek, Dimension-six terms in the standard model Lagrangian, JHEP 10 (2010) 085 [arXiv: 1008.4884] [INSPIRE].

[104] E.E. Jenkins, A.V. Manohar and M. Trott, Renormalization group evolution of the standard model dimension six operators I: formalism and lambda dependence, JHEP 10 (2013) 087 [arXiv: 1308.2627] [INSPIRE].

[105] E.E. Jenkins, A.V. Manohar and M. Trott, Renormalization group evolution of the standard model dimension six operators II: Yukawa dependence, JHEP 01 (2014) 035 [arXiv: 1310.4838] [INSPIRE].

[106] R. Alonso, E.E. Jenkins, A.V. Manohar and M. Trott, Renormalization group evolution of the standard model dimension six operators III: gauge coupling dependence and phenomenology, JHEP 04 (2014) 159 [arXiv: 1312.2014] [INSPIRE].

[107] H. Simma, Equations of motion for effective Lagrangians and penguins in rare B decays, $Z$. Phys. C 61 (1994) 67 [hep-ph/9307274] [INSPIRE]. 